\title{
Hydroxypyridyl Imines: Enhancing Chromatographic Separation and Stereochemical Analysis of Chiral Amines via Circular Dichroism
}

\author{
Leo A. Joyce*, Erik L. Regalado, Christopher J. Welch
}

Supporting Information: Table of Contents

- Instrumentation and Stationary Phases

S2

- SFC Screening Chromatograms

- Cyclohexylethylamine (CEA) alone

S3-S9

- CEA with HCA S10-S15

- 1-Methylbenzylamine (MBA) alone

$\mathrm{S} 16-\mathrm{S} 22$

- MBA with HCA

S23-S29

- 1-methyl-3-phenylpropylamine (MPP) alone

S30-S36

- MPP with HCA

S37-S45

- 1-aminoheptane (AMH) with HCA

S44-S50

- 4-fluoro-1-methylbenzylamine with HCA

S51-S57

- 4-chloro-1-methylbenzylamine with HCA

- 4-methoxy-1-methylbenzylamine with HCA

- Hydrolysis monitoring 


\section{Instrumentation}

Chiral SFC screening and optimization experiments were carried out on Waters Acquity UPC ${ }^{2}$ (WatersCorp., Milford, MA, USA) systems equipped with a fluid delivery module (a liquid $\mathrm{CO}_{2}$ pump and a modifier pump), a sampler manager (FL autosampler), two auxiliary column managers allowing six installed columns, a photodiode array detector, and MassLynx software. $S F C-U V-C D$ experiments were performed on an Agilent 1100 with an Aurora A5 Fusion SFC module. The Agilent system comprised a modified G1322A vacuum degasser, a modified G1312A binary pump, a G1367A WPALS autosampler, a G1316A column compartment, a G1315B diode array detector and a CD-1595 circular dichroism detector (Jasco, Easton, MD). The system was controlled by Chemstation software. Reversed phase HPLC-UV$C D$ analyzes were carried out on an Agilent 1100 system (Agilent Technologies, Palo Alto, CA, USA). The Agilent system comprised a G1312A binary pump, a G1379A degasser, a G1367A WPALS autosampler, a G1316A column compartment, a G1315B diode array detector, and a CD-1595 detector. Standalone CD spectra were measured on a Jasco 810 CD spectropolarimeter equipped with a $0.1 \mathrm{~cm}$ microcell and a thermostat $\left(25^{\circ} \mathrm{C}\right)$.

\section{Stationary Phases}

Columns packed with Chiralpak (AD-3, AS-3, IA, IB, IC, IC, IE, IF) and Chiralcel (OD-3, OJ-3, OZ-3) were purchased from Chiral Technologies (West Chester, PA, USA); Lux (Amylose-2 and Cellulose-4) columns were purchased from Phenomenex (Torrance, CA, USA). Dimensions and particle size for all columns were $4.6 \mathrm{~mm} \mathrm{I.D.} \times 150 \mathrm{~mm}, 3 \mu \mathrm{m}$. 

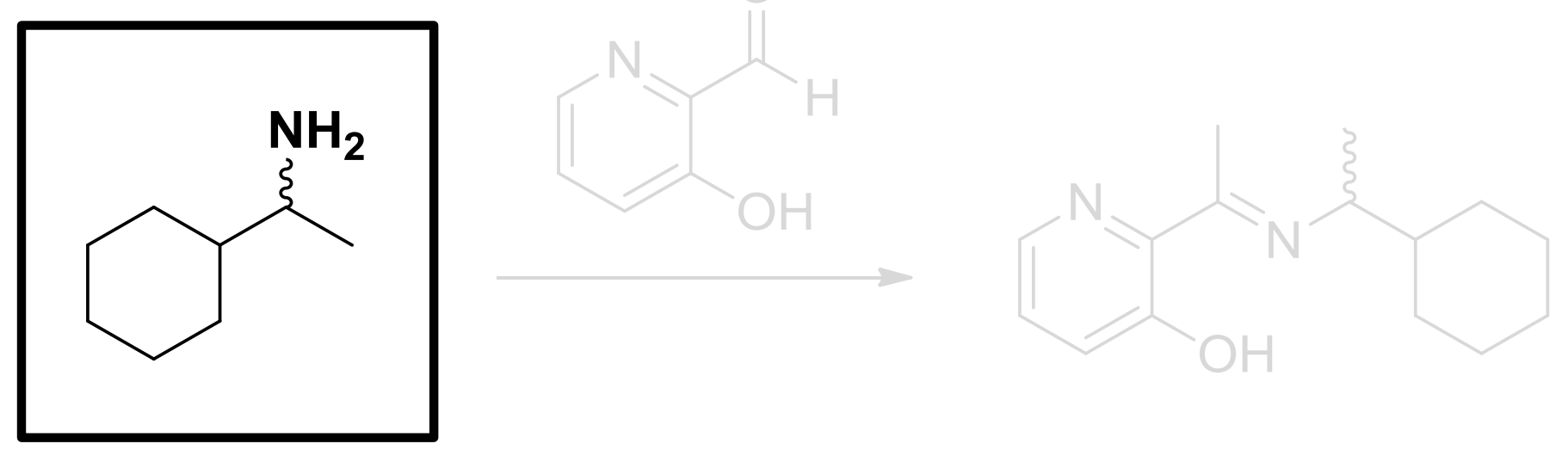

Primary Chiral SFC Screen

12 columns, 3 solvents

(with and without $25 \mathrm{mM}$ IBA) 


\section{SFC Screening: 1-40\% (0-5 min), 40\% (5-6 min), no post. $3 \mathrm{~mL} / \mathrm{min}, 200 \mathrm{bar}, 40^{\circ} \mathrm{C}$}

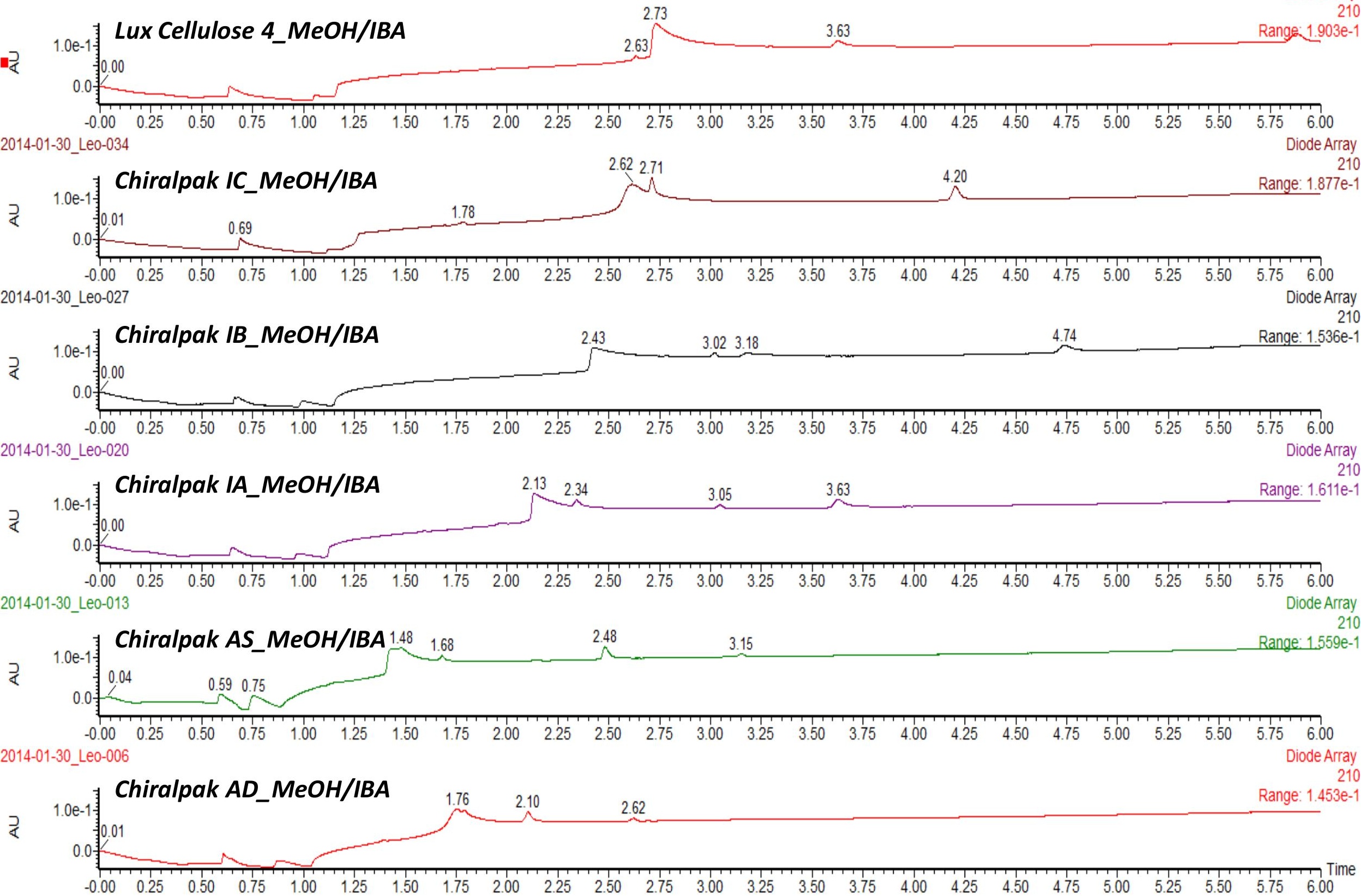


SFC Screening: $1-40 \%(0-5 \mathrm{~min}), 40 \%(5-6 \mathrm{~min})$, no post. $3 \mathrm{~mL} / \mathrm{min}, 200 \mathrm{bar}, 40^{\circ} \mathrm{C}$
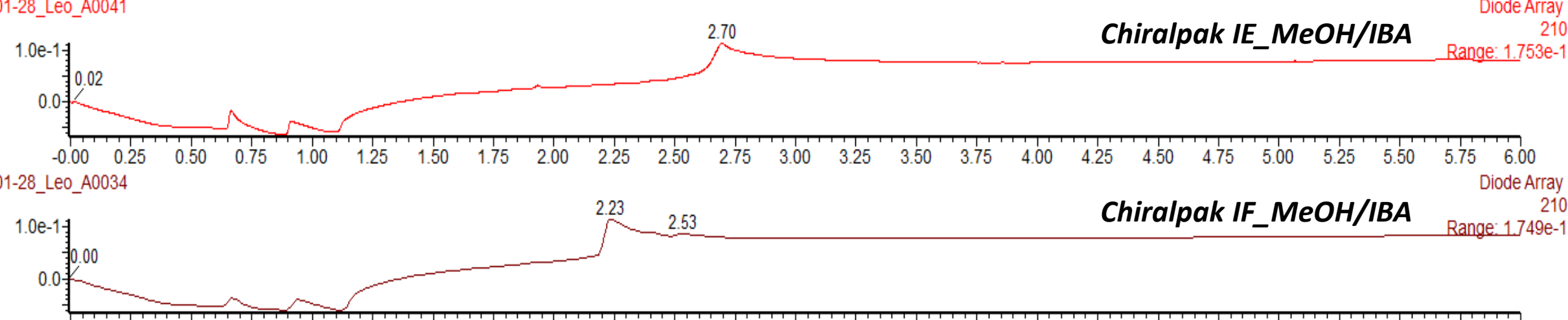

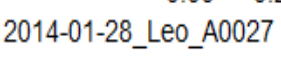

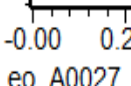

₹

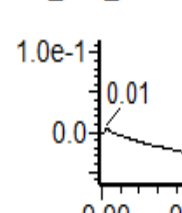

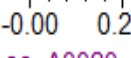

2014-01-28_LeO_A0020

২

1.0e-1)

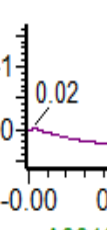

$\overbrace{}^{2.23} 2.53$

Chiralpak IF_MeOH/IBA

210

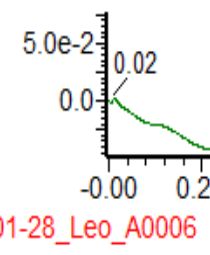

२

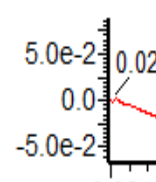

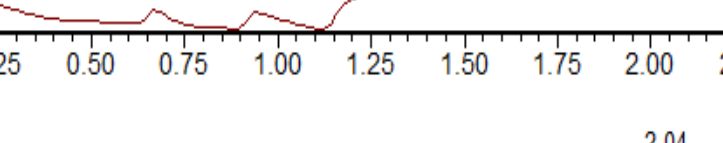
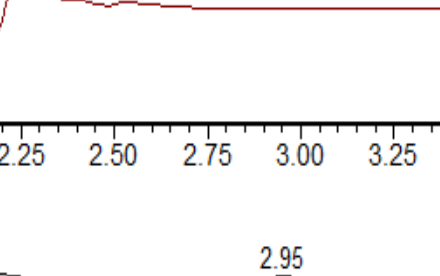

2.95 Lux Amylose 2_MeOH/IBA Range: $1769 \mathrm{e}-1$

6.00

210
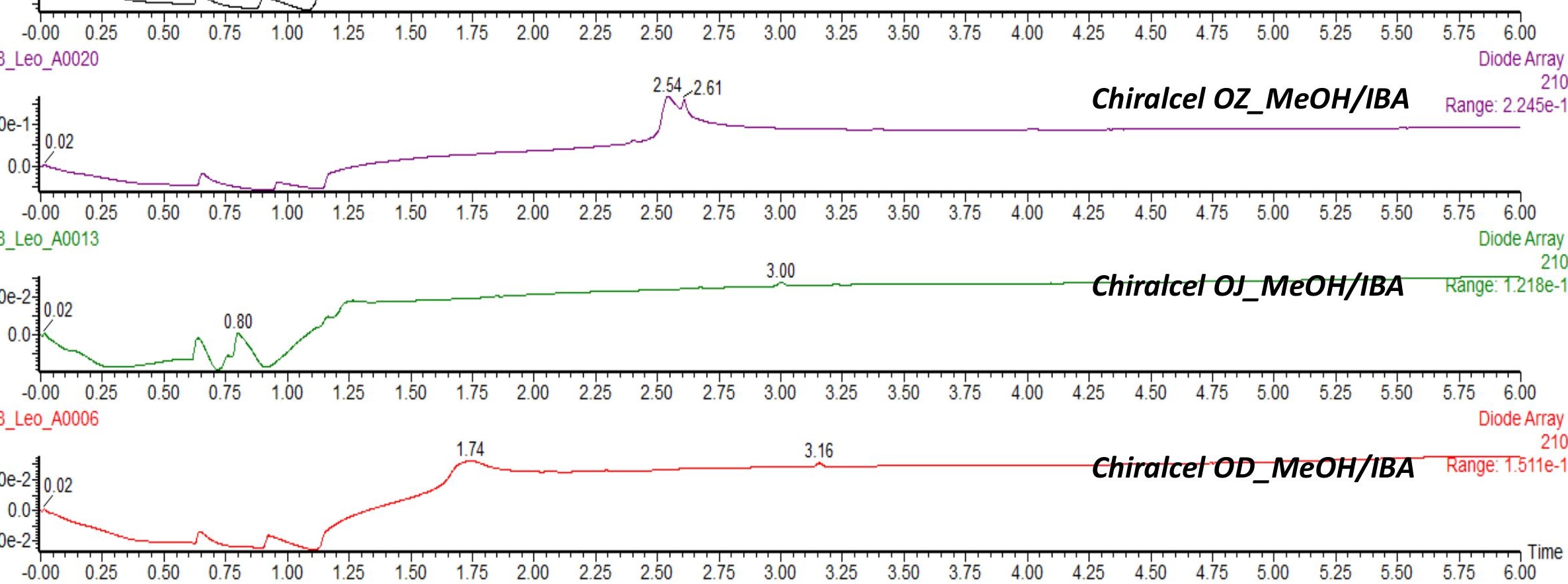
SFC Screening: $1-40 \%(0-5 \mathrm{~min}), 40 \%(5-6 \mathrm{~min})$, no post. $3 \mathrm{~mL} / \mathrm{min}, 200 \mathrm{bar}, 40^{\circ} \mathrm{C}$

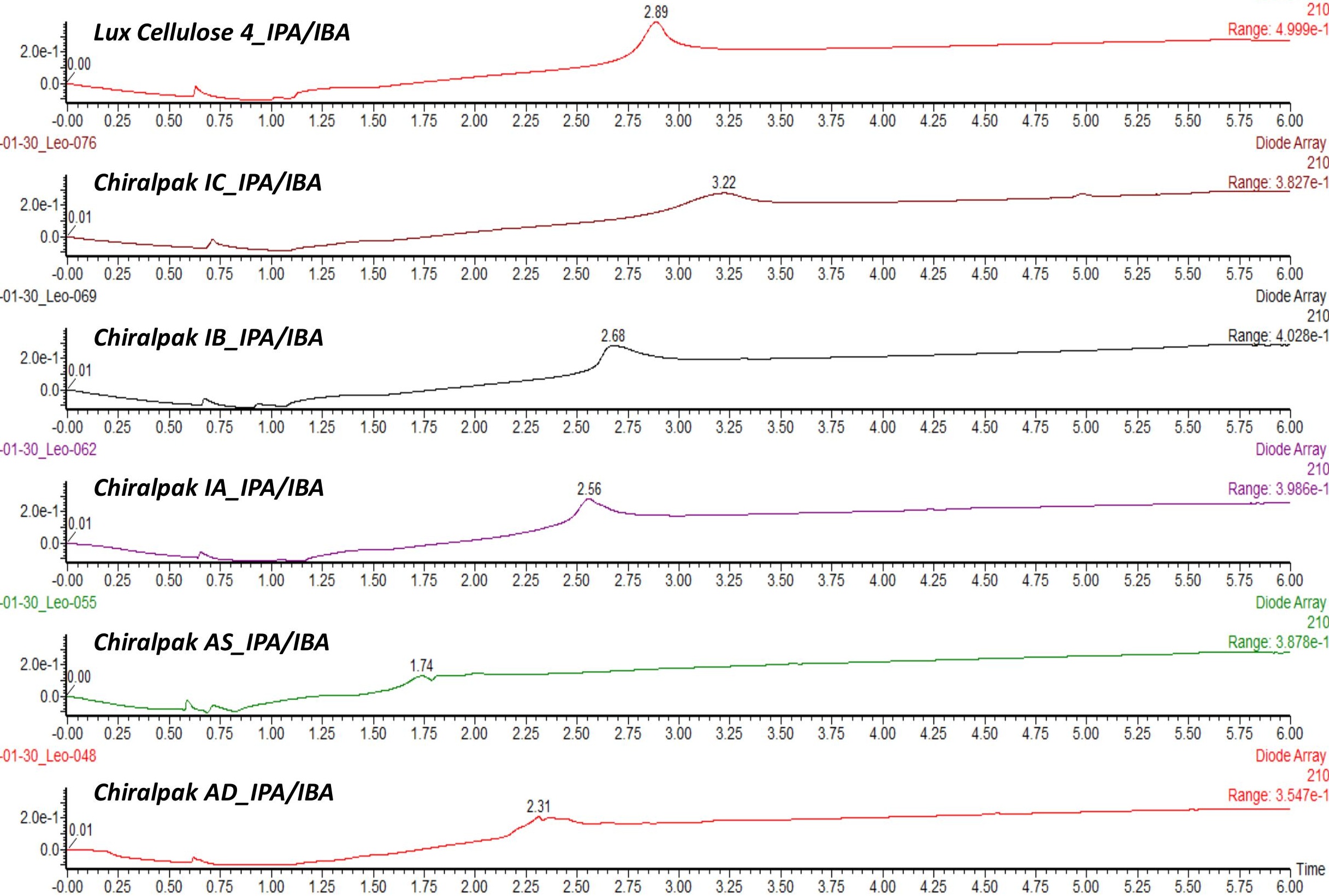




\section{SFC Screening: 1-40\% (0-5 min), 40\% (5-6 min), no post. $3 \mathrm{~mL} / \mathrm{min}, 200 \mathrm{bar}, 40^{\circ} \mathrm{C}$}

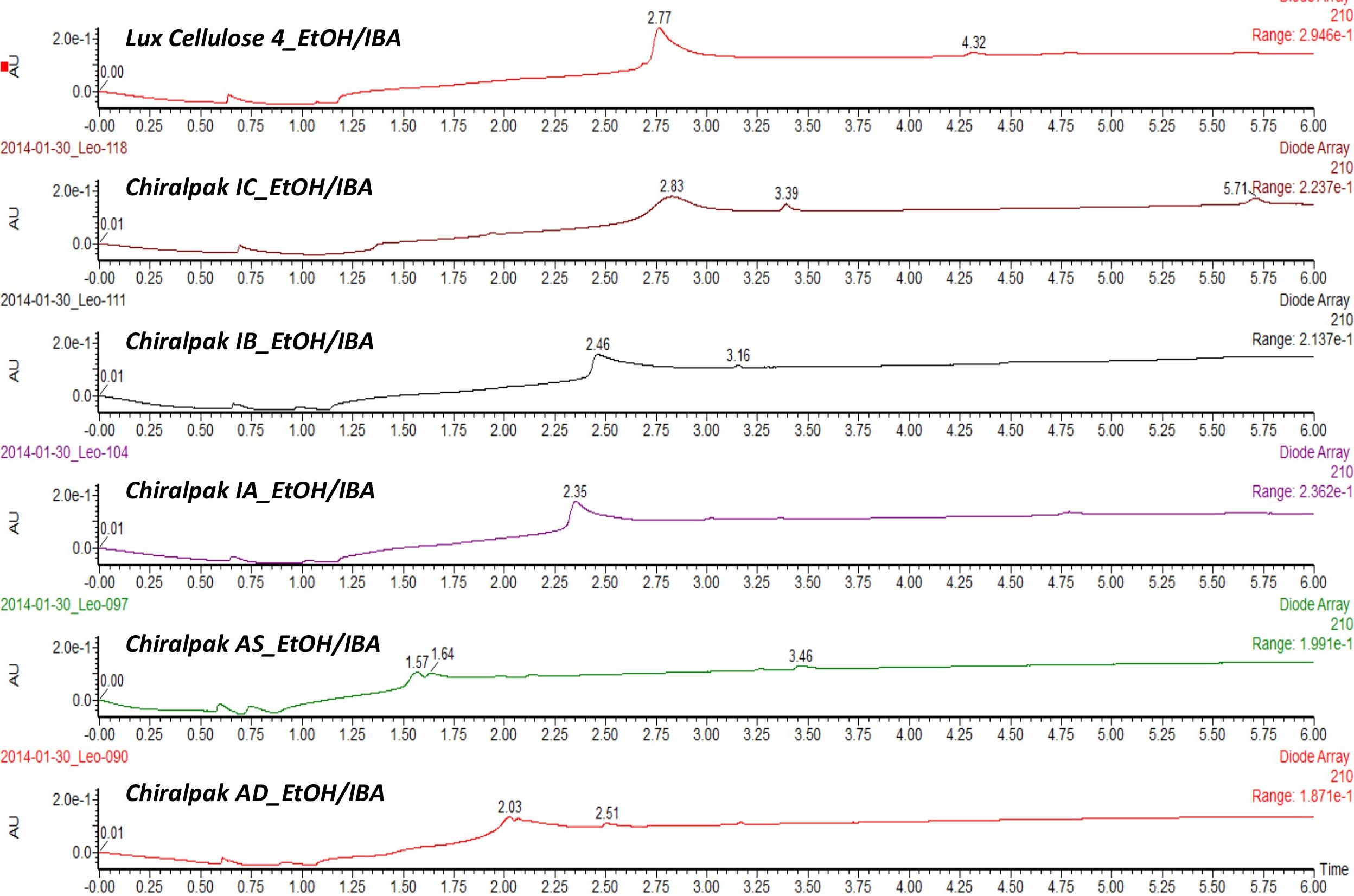


SFC Screening: 1-40\% (0-5 min), 40\% (5-6 min), no post. $3 \mathrm{~mL} / \mathrm{min}, 200 \mathrm{bar}, 40^{\circ} \mathrm{C}$
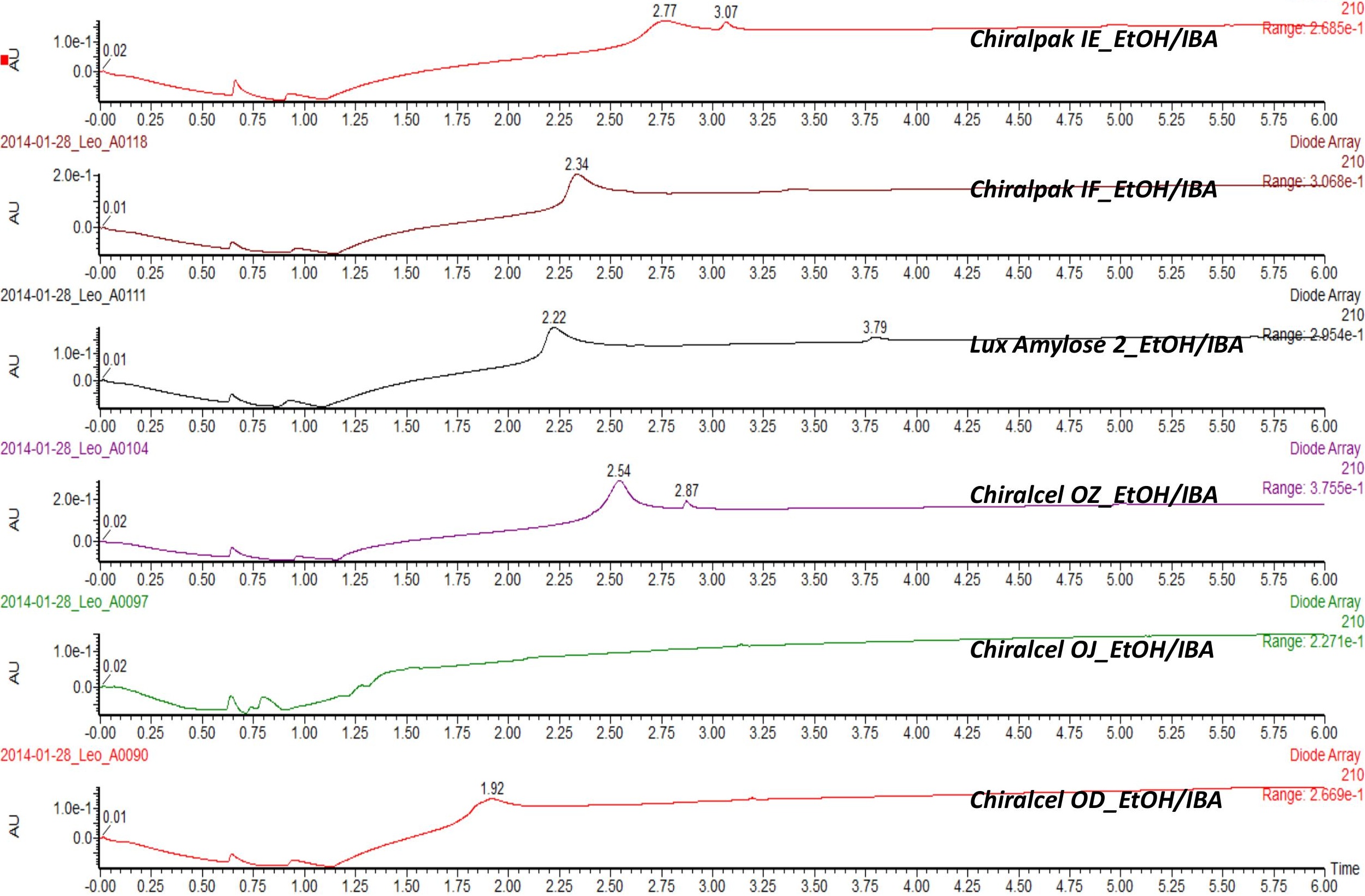


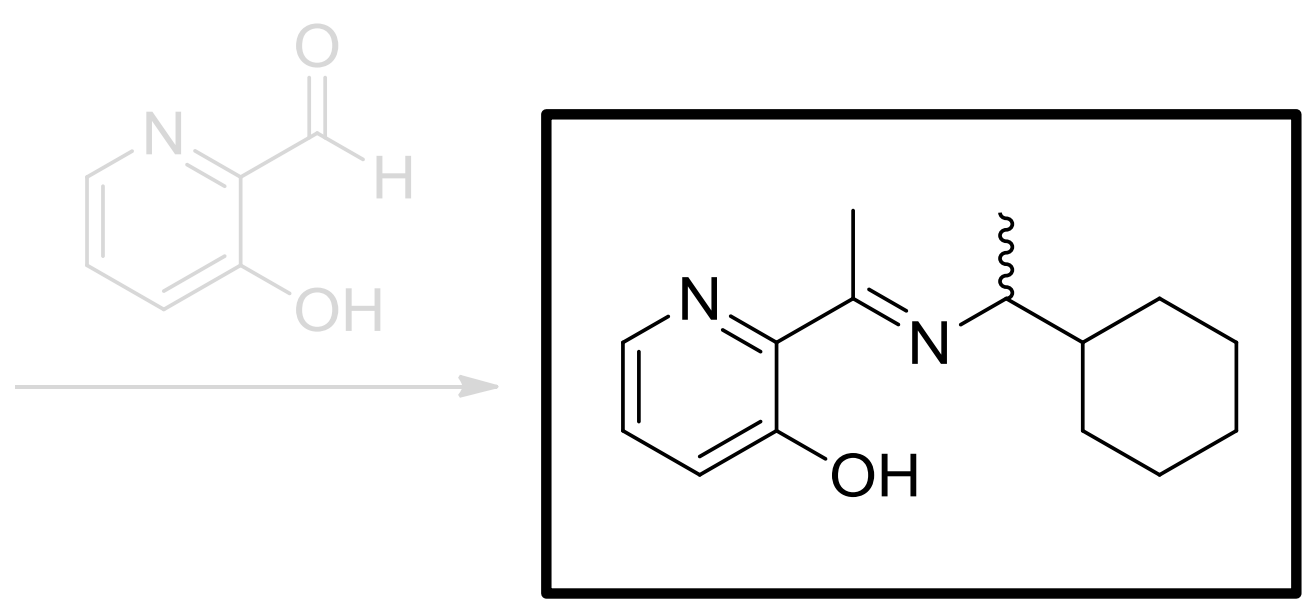

Primary Chiral SFC Screen

12 columns, 3 solvents

(with and without 25 mM IBA) 
SFC Screening: $1-40 \%(0-5 \mathrm{~min}), 40 \%(5-6 \mathrm{~min})$, no post. $3 \mathrm{~mL} / \mathrm{min}, 200 \mathrm{bar}, 40^{\circ} \mathrm{C}$

S11
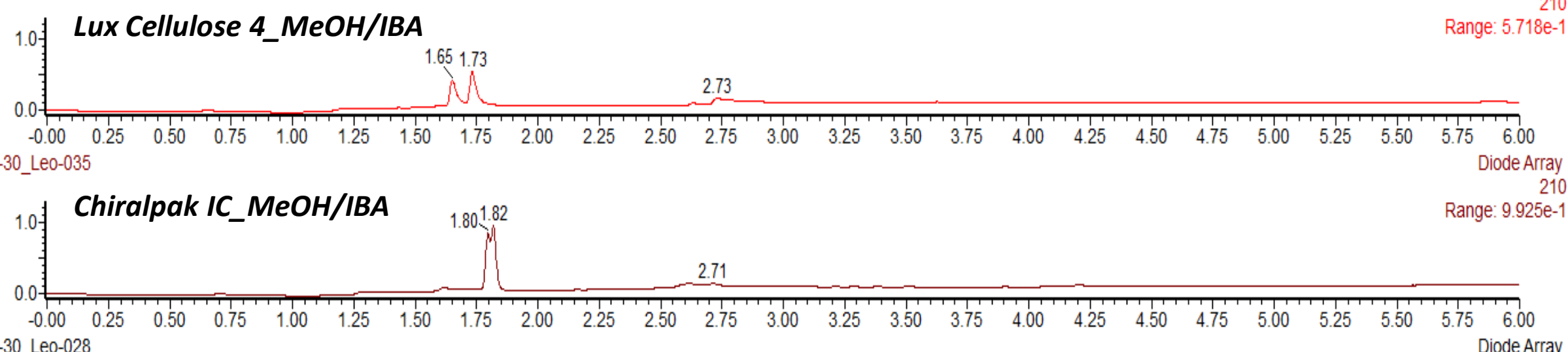

2014-01-30 Leo-028
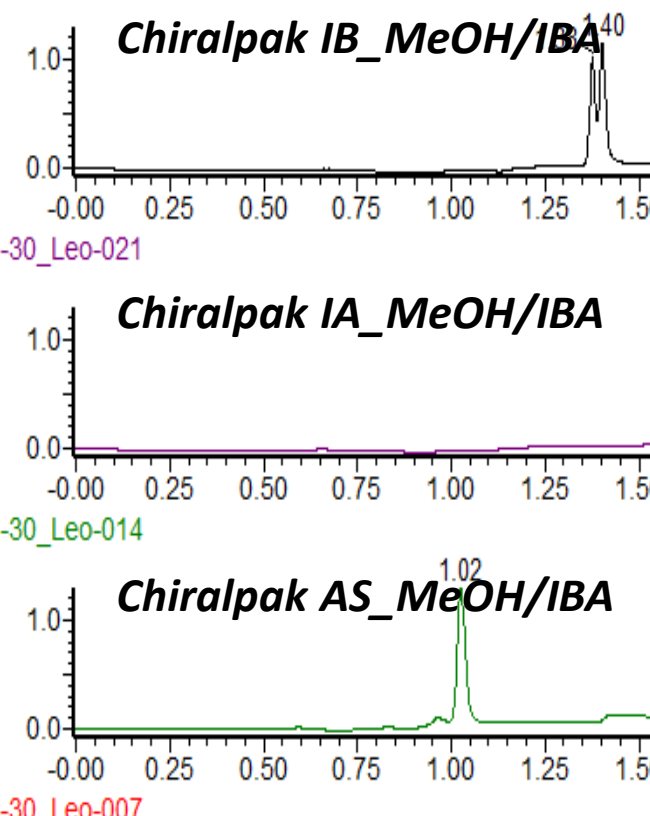

ج

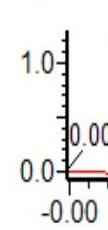

Chiralpak AD_MeOH/IBA

00

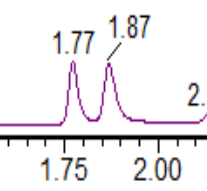

Range: $9.925 \mathrm{e}-1$

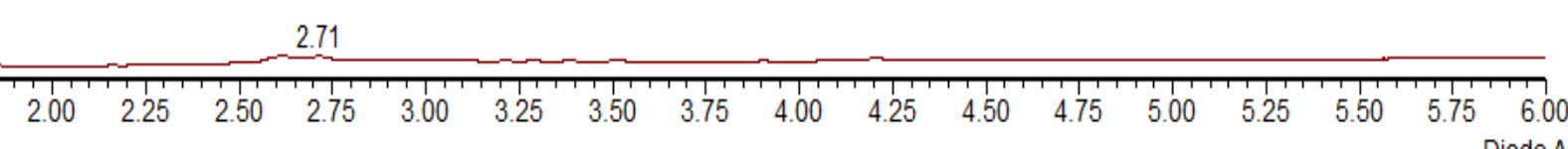

Diode Array

Range: 1.174

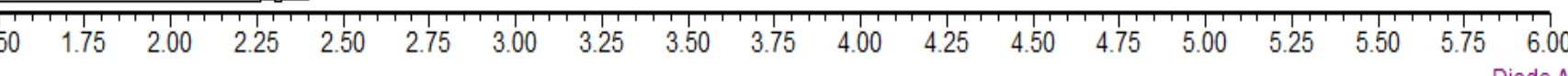

Diode Array

Range: $6.618 \mathrm{e}-1$

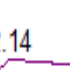

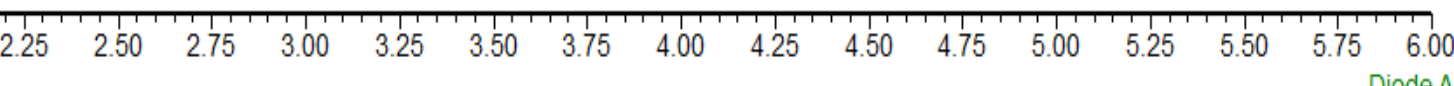

Diode Array

Range: 1.319 
SFC Screening: 1-40\% (0-5 min), 40\% (5-6 min), no post. $3 \mathrm{~mL} / \mathrm{min}, 200 \mathrm{bar}, 40^{\circ} \mathrm{C}$

S12 CEA Imine_4

Diode Array
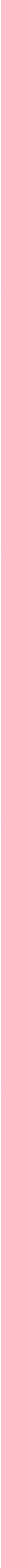
SFC Screening: $1-40 \%(0-5 \mathrm{~min}), 40 \%(5-6 \mathrm{~min})$, no post. $3 \mathrm{~mL} / \mathrm{min}, 200 \mathrm{bar}, 40^{\circ} \mathrm{C}$
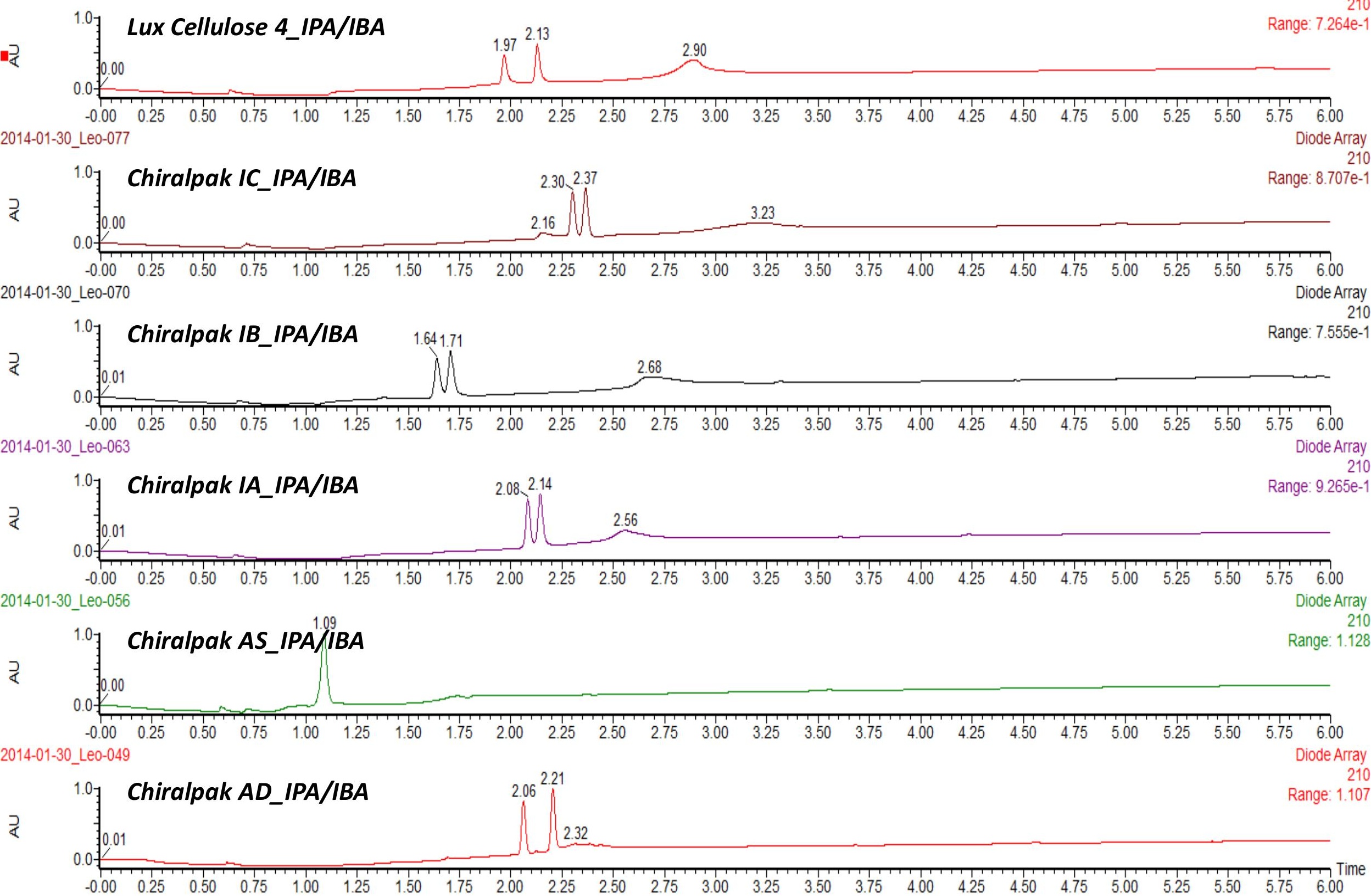
SFC Screening: 1-40\% (0-5 min), 40\% (5-6 min), no post. $3 \mathrm{~mL} / \mathrm{min}, 200 \mathrm{bar}, 40^{\circ} \mathrm{C}$

S14

CEA Imine_4
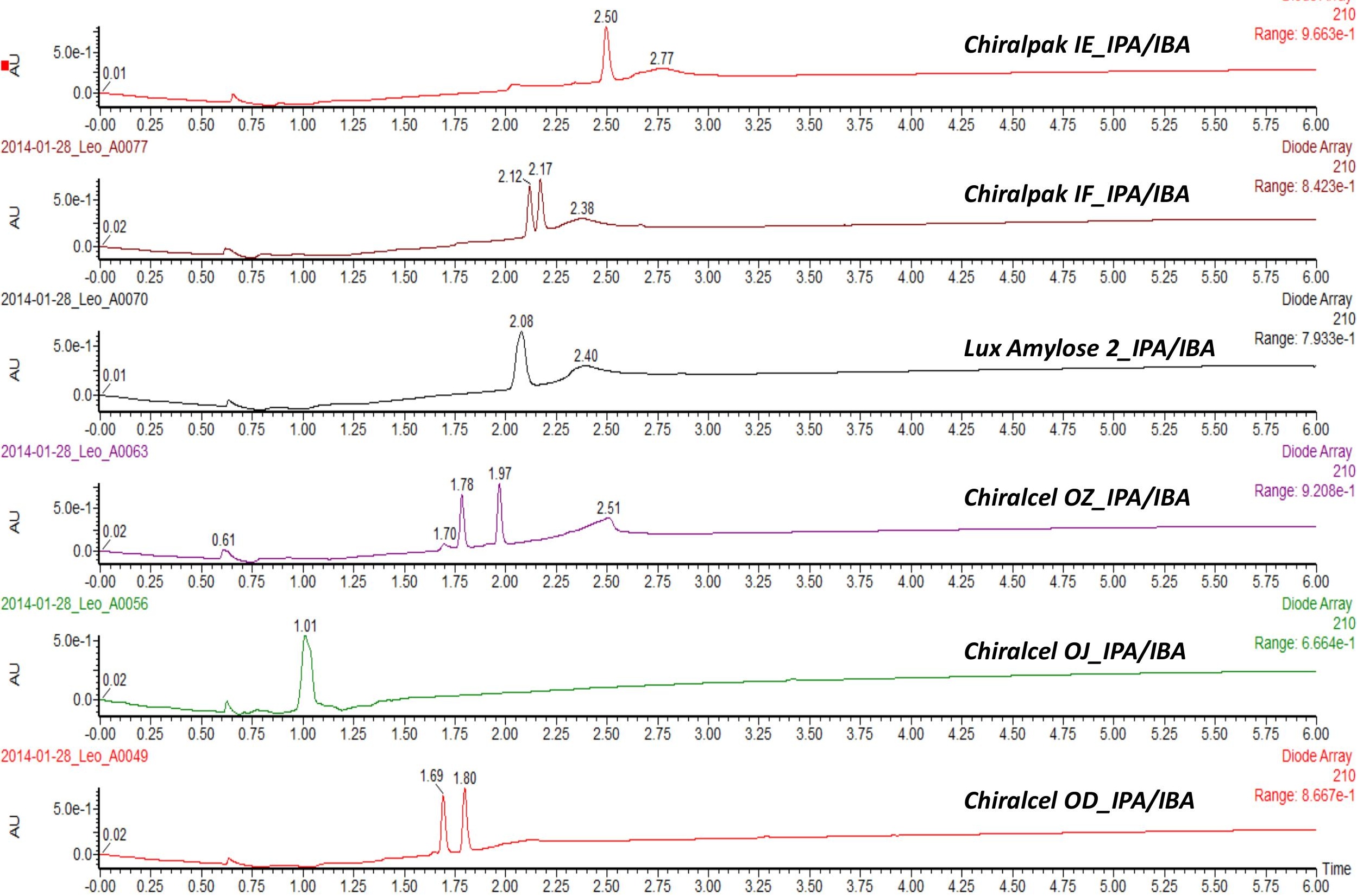
SFC Screening: $1-40 \%(0-5 \mathrm{~min}), 40 \%(5-6 \mathrm{~min})$, no post. $3 \mathrm{~mL} / \mathrm{min}, 200 \mathrm{bar}, 40^{\circ} \mathrm{C}$
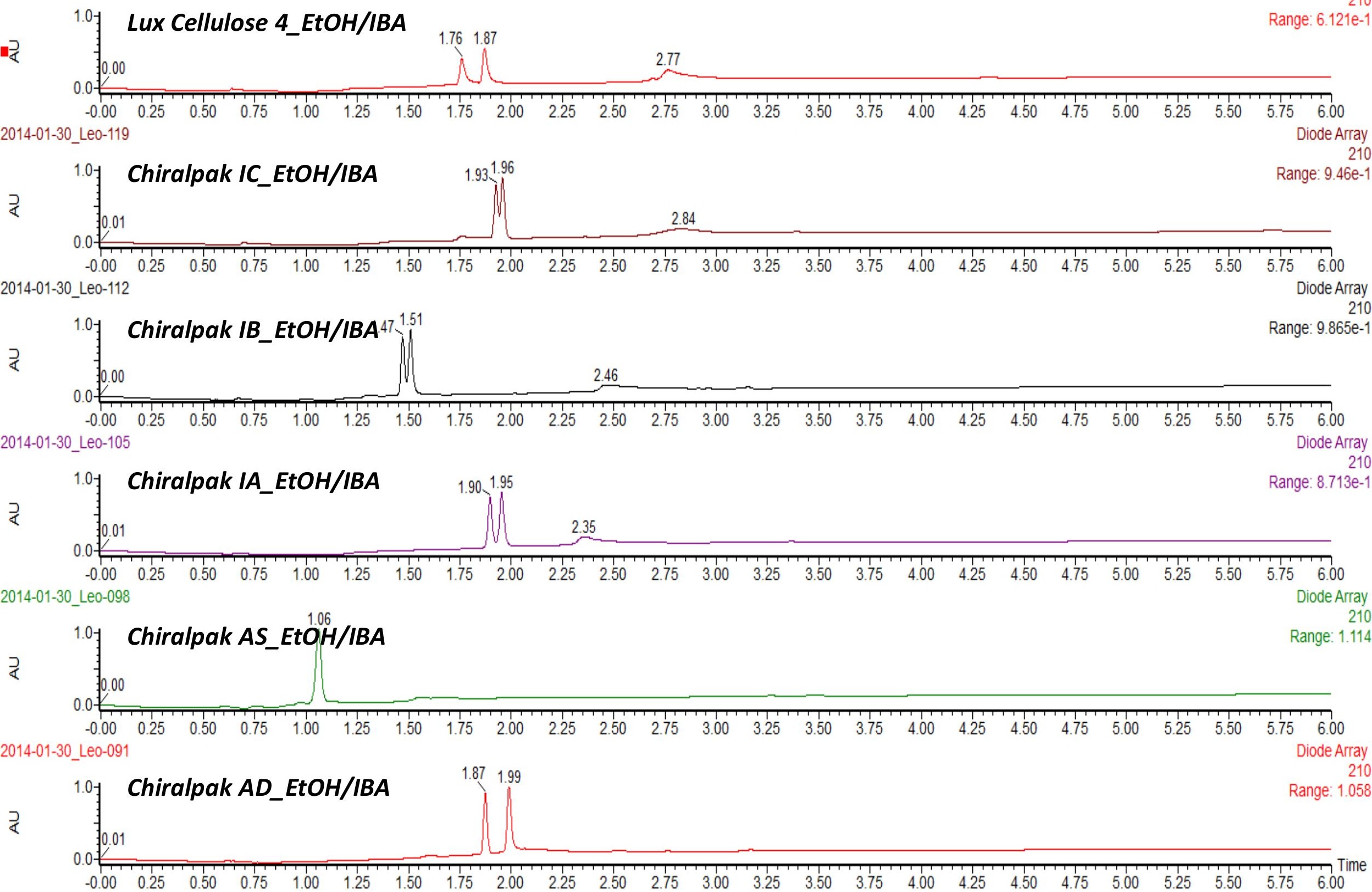

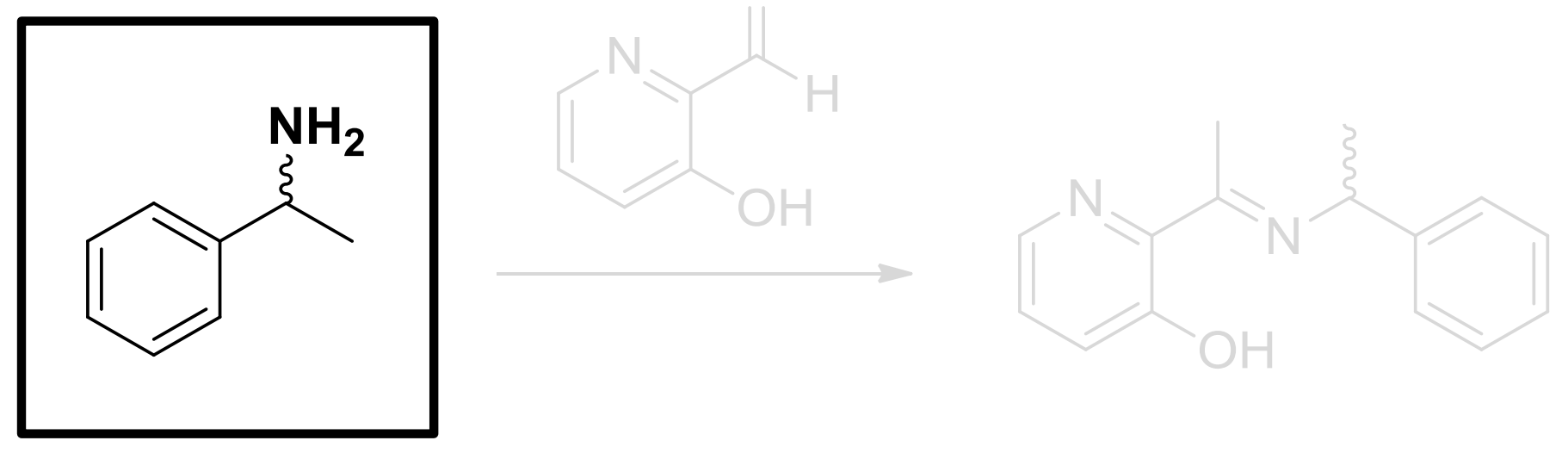

Primary Chiral SFC Screen

12 columns, 3 solvents

(with and without $25 \mathrm{mM}$ IBA) 


\section{SFC Screening: 1-40\% (0-5 min), 40\% (5-6 min), no post. $3 \mathrm{~mL} / \mathrm{min}, 200 \mathrm{bar}, 40^{\circ} \mathrm{C}$}

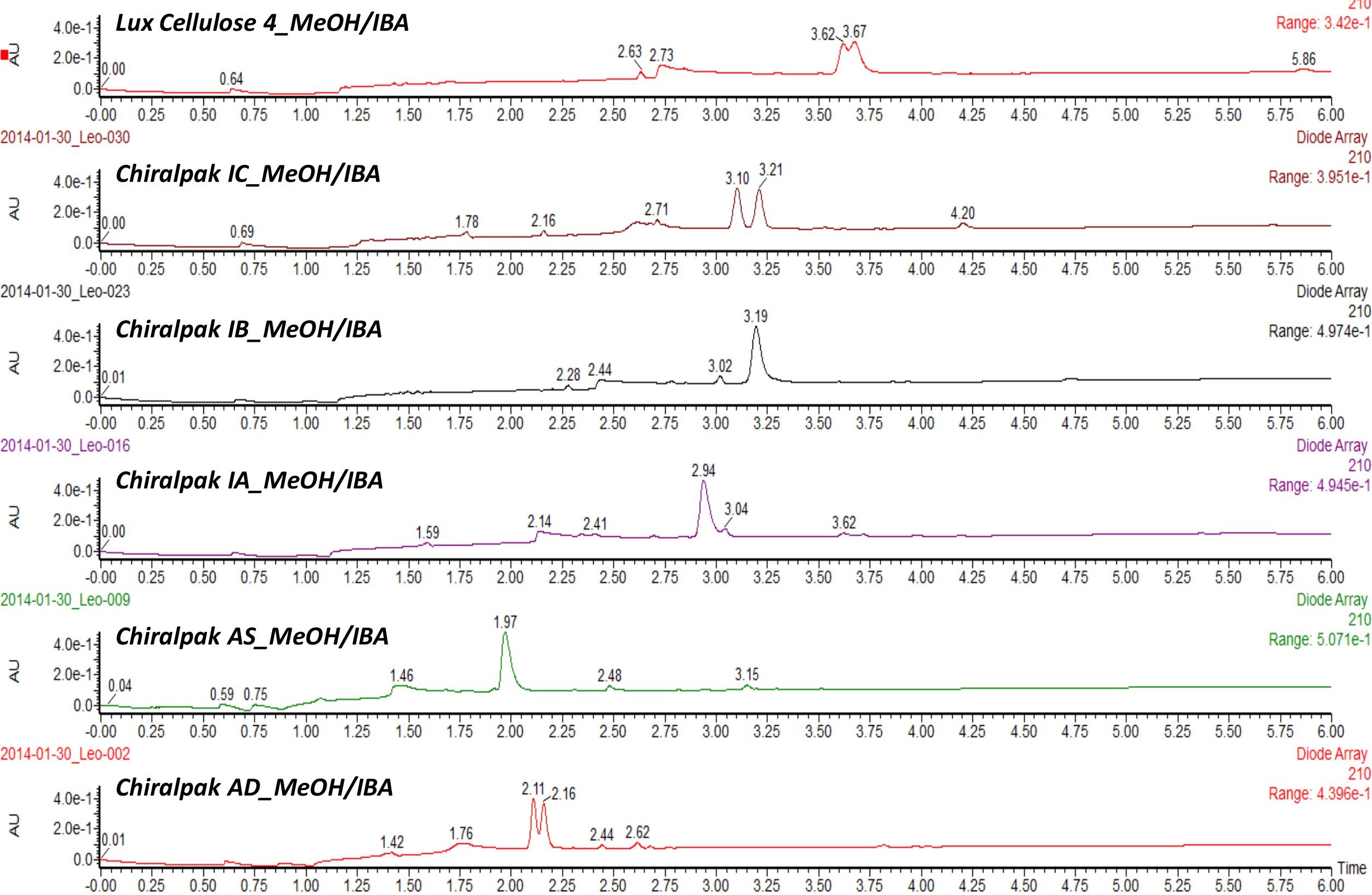


SFC Screening: 1-40\% (0-5 min), 40\% (5-6 min), no post. $3 \mathrm{~mL} / \mathrm{min}, 200 \mathrm{bar}, 40^{\circ} \mathrm{C}$

2014-01-28_Leo_A0037

MBA Amine_1
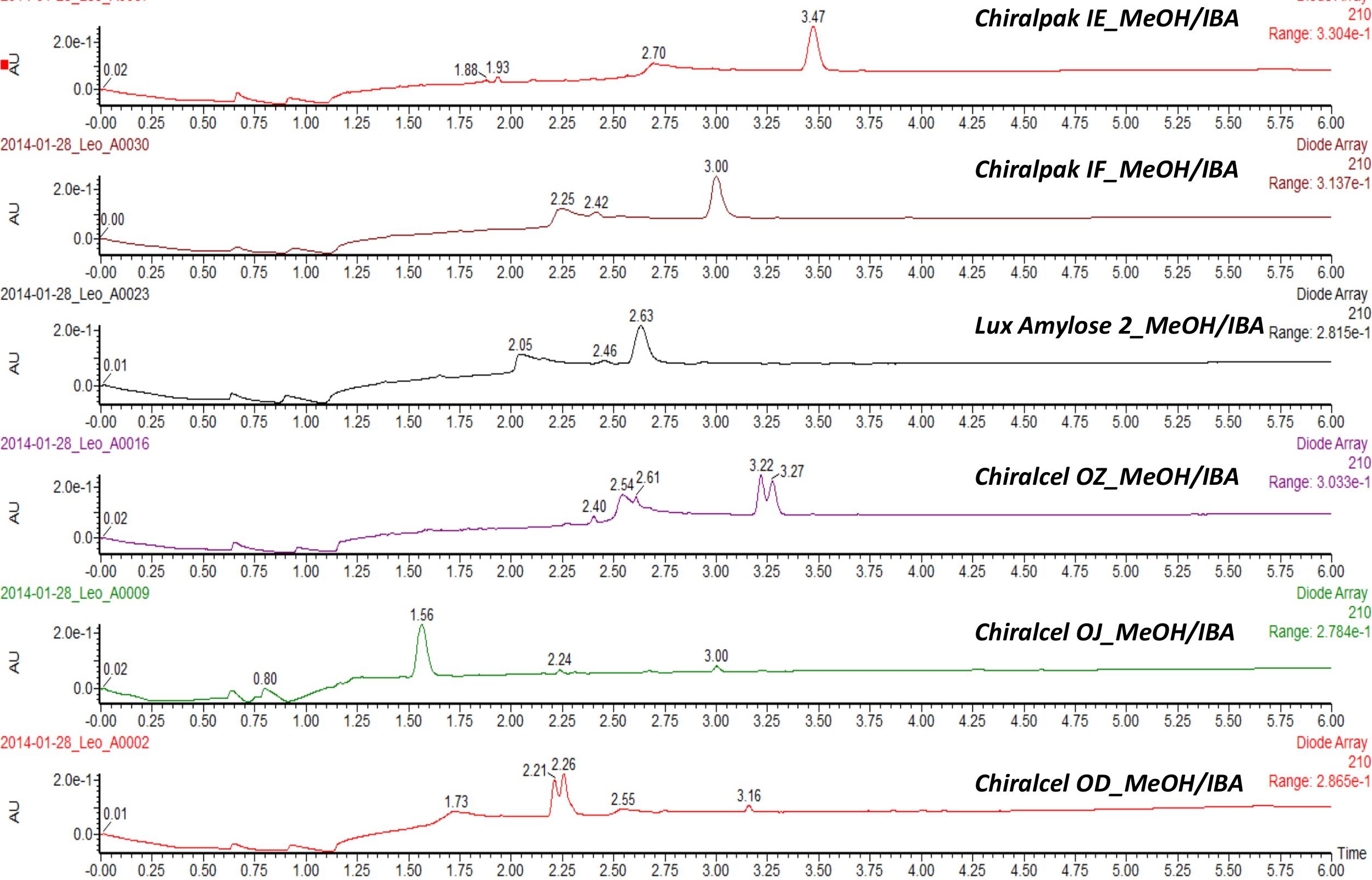


\section{SFC Screening: 1-40\% (0-5 min), 40\% (5-6 min), no post. $3 \mathrm{~mL} / \mathrm{min}, 200 \mathrm{bar}, 40^{\circ} \mathrm{C}$}

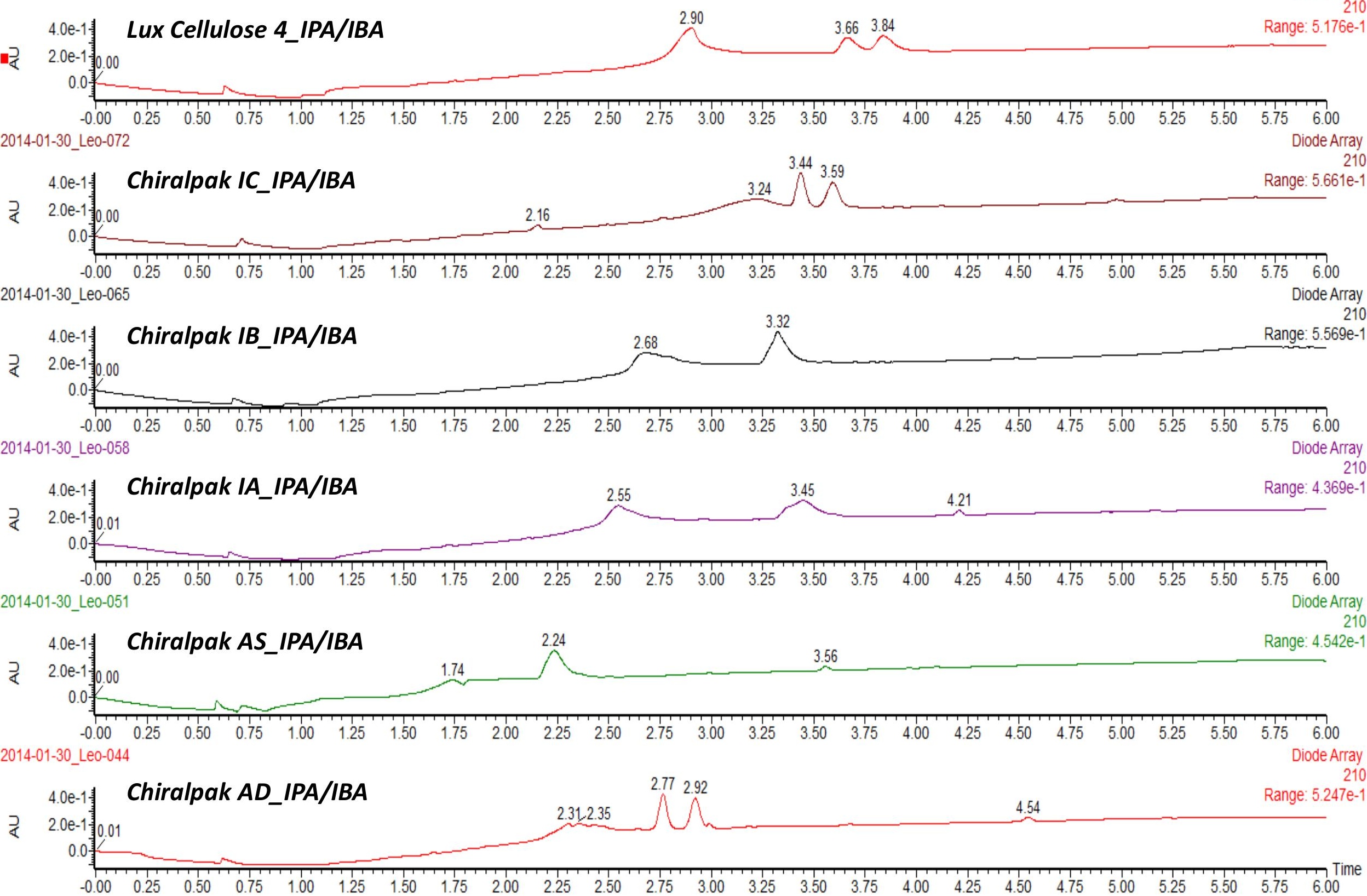


SFC Screening: $1-40 \%(0-5 \mathrm{~min}), 40 \%(5-6 \mathrm{~min})$, no post. $3 \mathrm{~mL} / \mathrm{min}, 200 \mathrm{bar}, 40^{\circ} \mathrm{C}$

S21
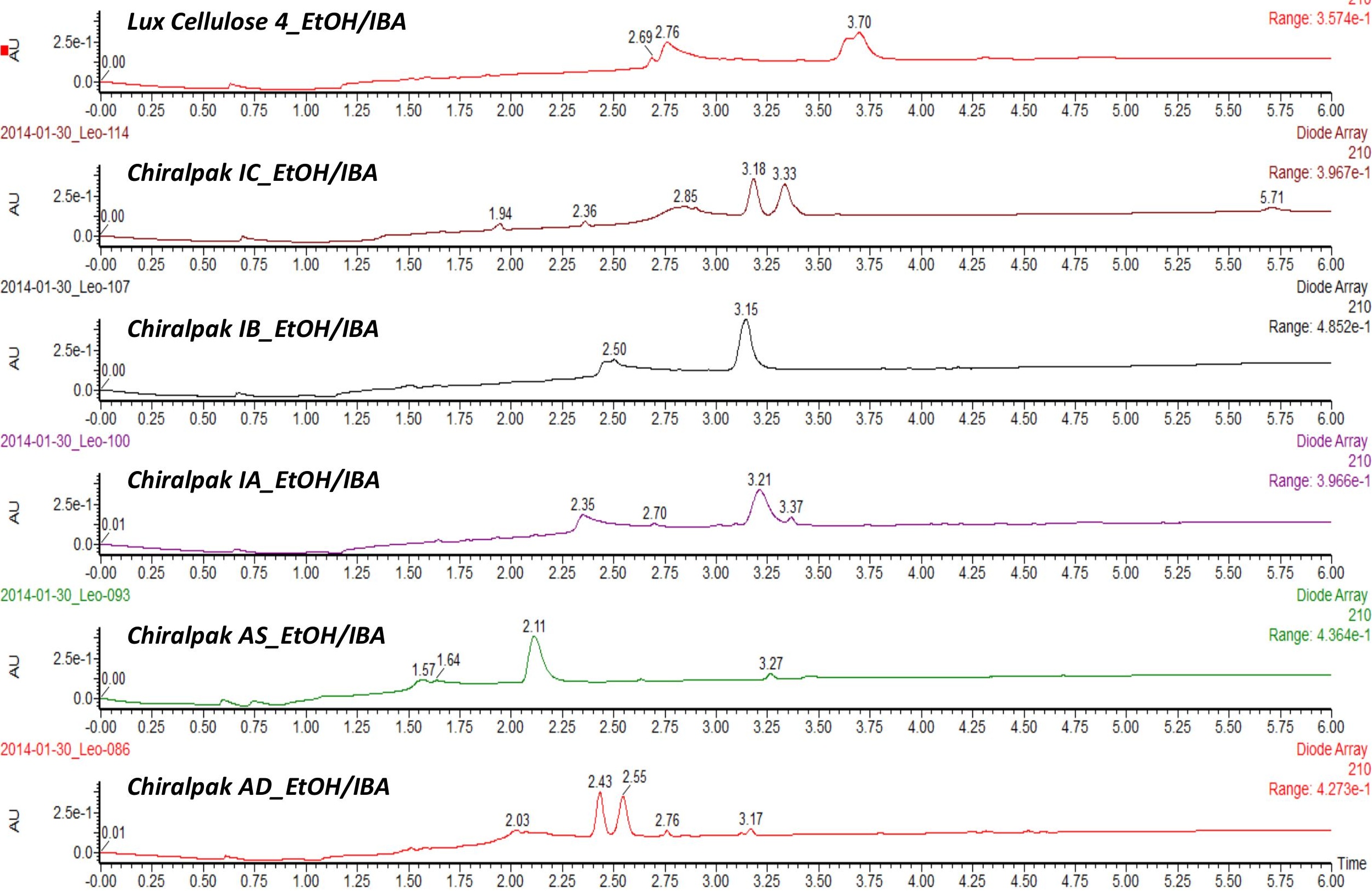
SFC Screening: 1-40\% (0-5 min), 40\% (5-6 min), no post. $3 \mathrm{~mL} / \mathrm{min}, 200 \mathrm{bar}, 40^{\circ} \mathrm{C}$

S22

MBA Amine_1

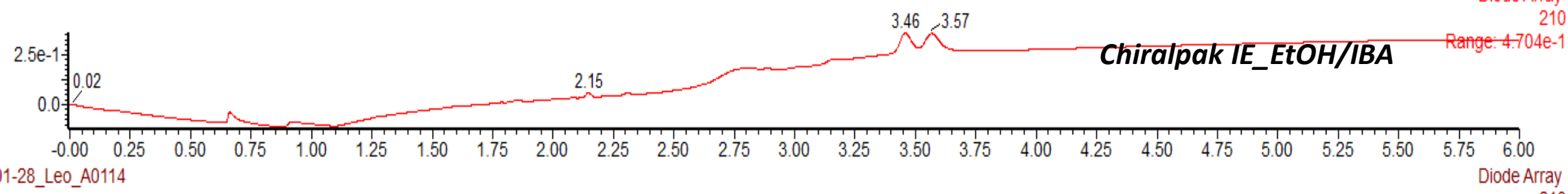

2014-01-28_Leo_A0114

2
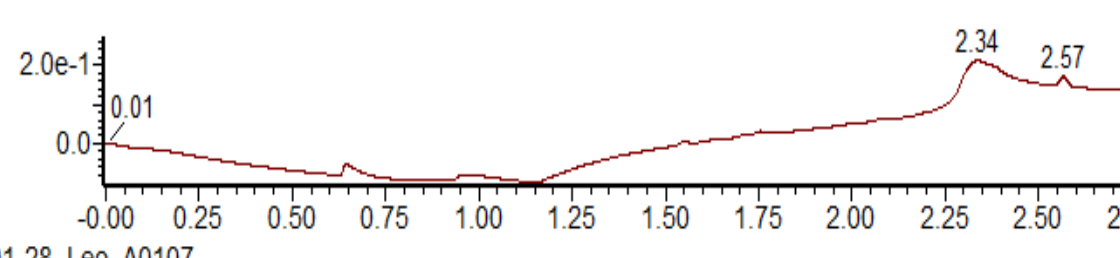

Chiralpak IF_EtOH/IBA

Range: $3.666 \mathrm{e}-1$ 014-01-28_Leo A0107

2
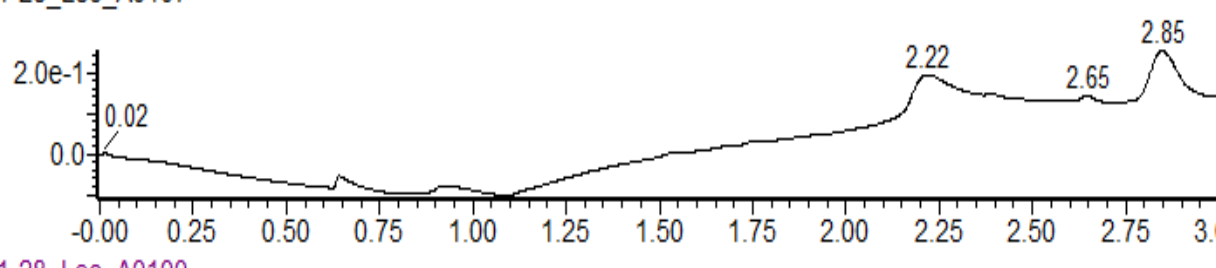

2014-01-28_Leo_A0100

₹
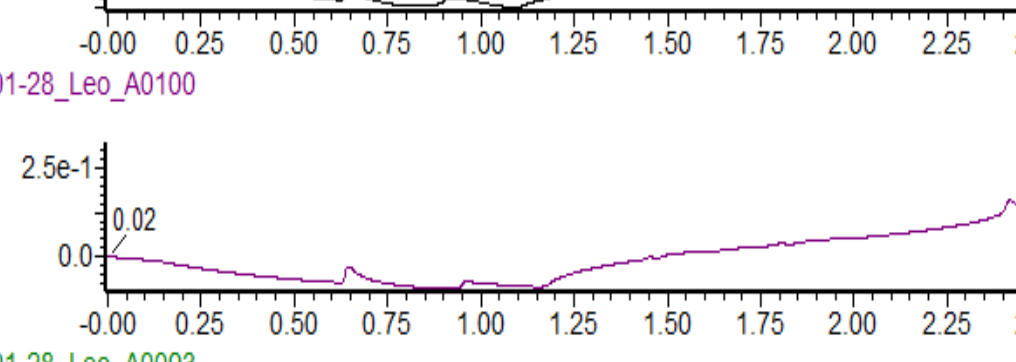

ج

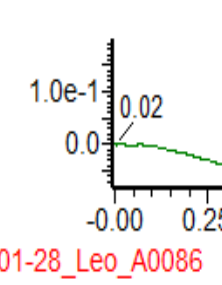
$\mathrm{A} 0093$
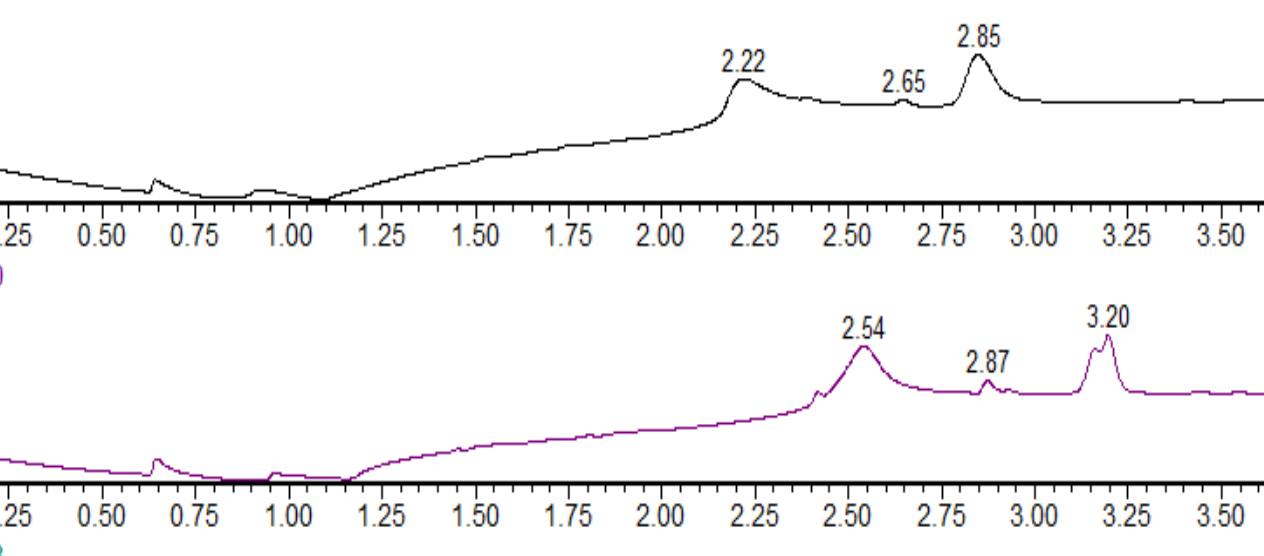

3.80

Lux Amylose 2_EtOH/IBA

6.00

210
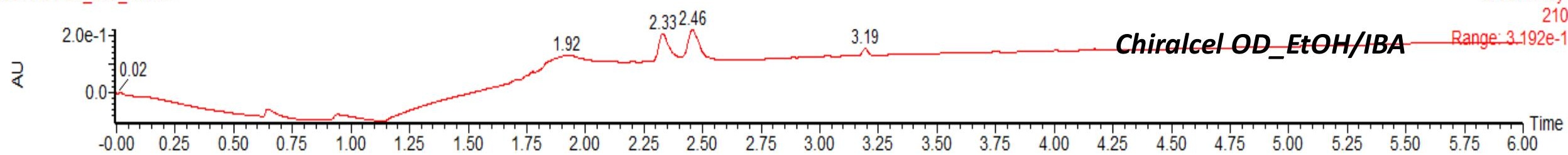

Chiralcel OD_EtOH/IBA_Range: $3192 \mathrm{e}-1$

Chiralcel OJ_EtOH/HA_Range: $2738 \mathrm{e}-1$ 


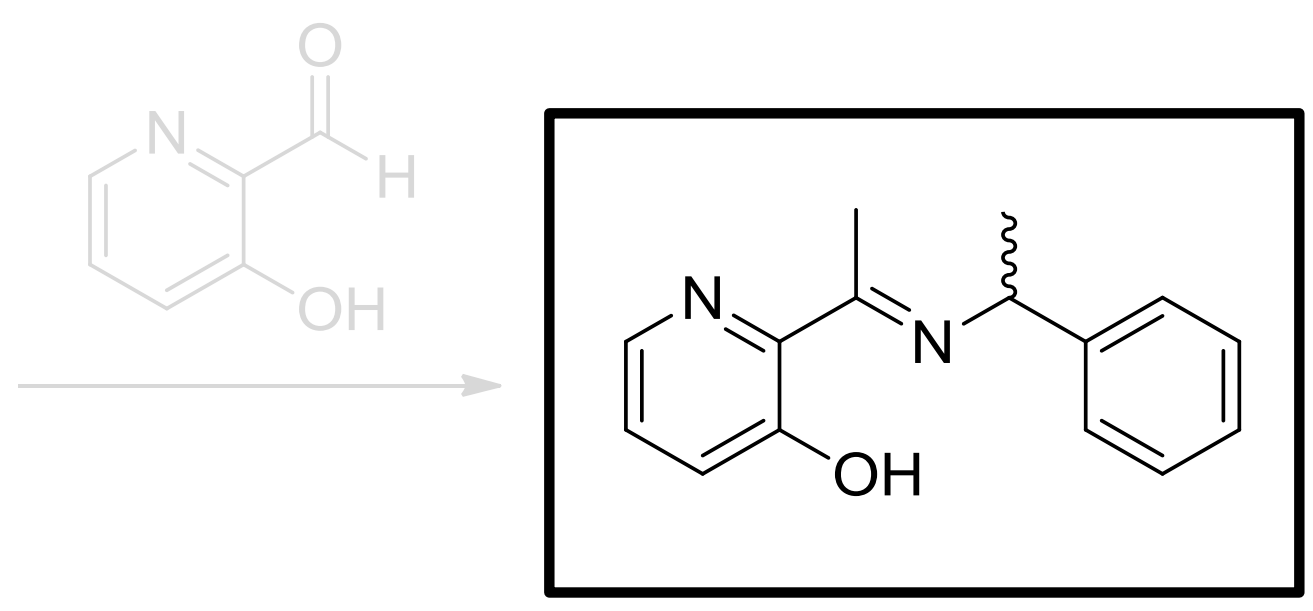

Primary Chiral SFC Screen

12 columns, 3 solvents

(with and without $25 \mathrm{mM}$ IBA) 
SFC Screening: $1-40 \%(0-5 \mathrm{~min}), 40 \%(5-6 \mathrm{~min})$, no post. $3 \mathrm{~mL} / \mathrm{min}, 200 \mathrm{bar}, 40^{\circ} \mathrm{C}$

1.0y Lux Cellulose 4_MeOH/IBA

Diode Array

Range: $2.045 \mathrm{e}-1$

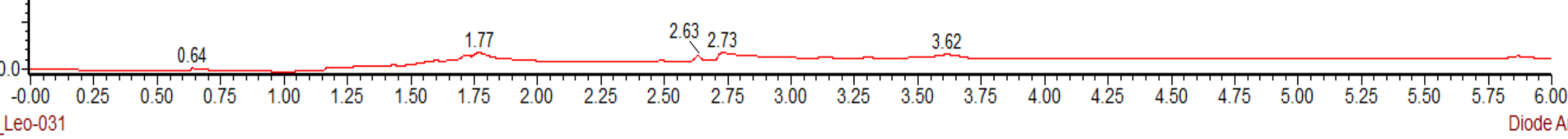
2014-01-30_Le0-031

${ }_{1.0}$ Chiralpak IC_MeOH/IBA

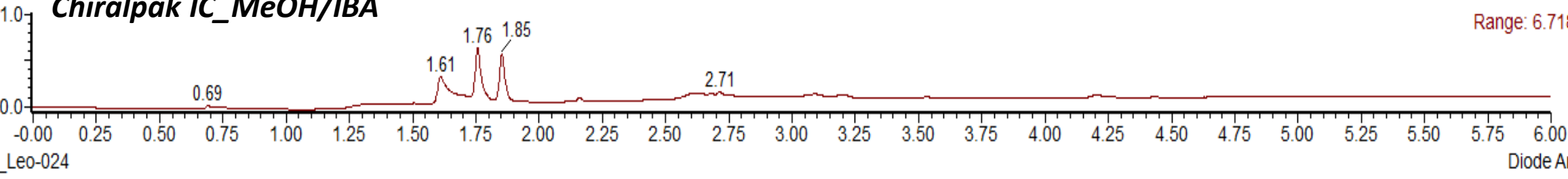
2014-01-30_Leo-024

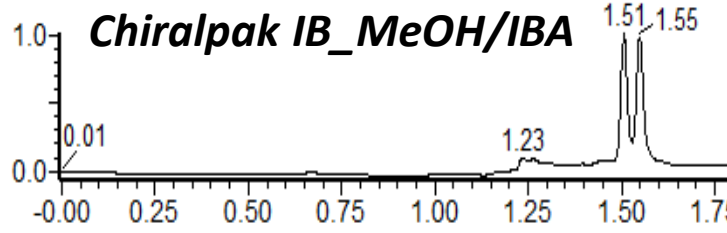
2014-01-30_Leo-017
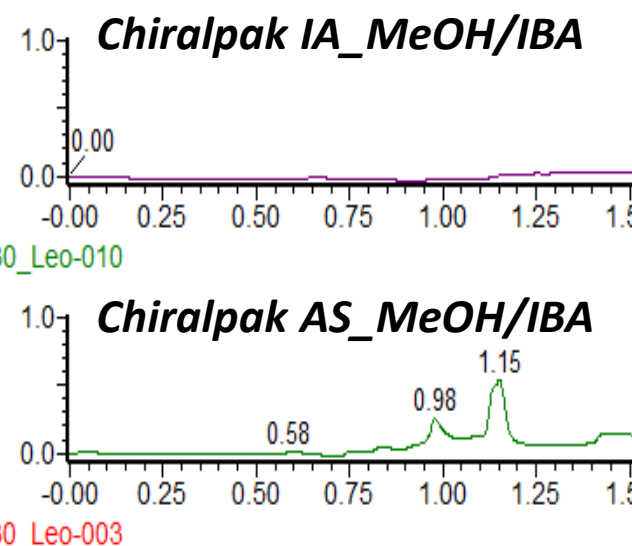

Chiralpak AS_MeOH/IBA

1.0

$0.0 \frac{\int_{-0.00}^{1.0}}{0.010}$
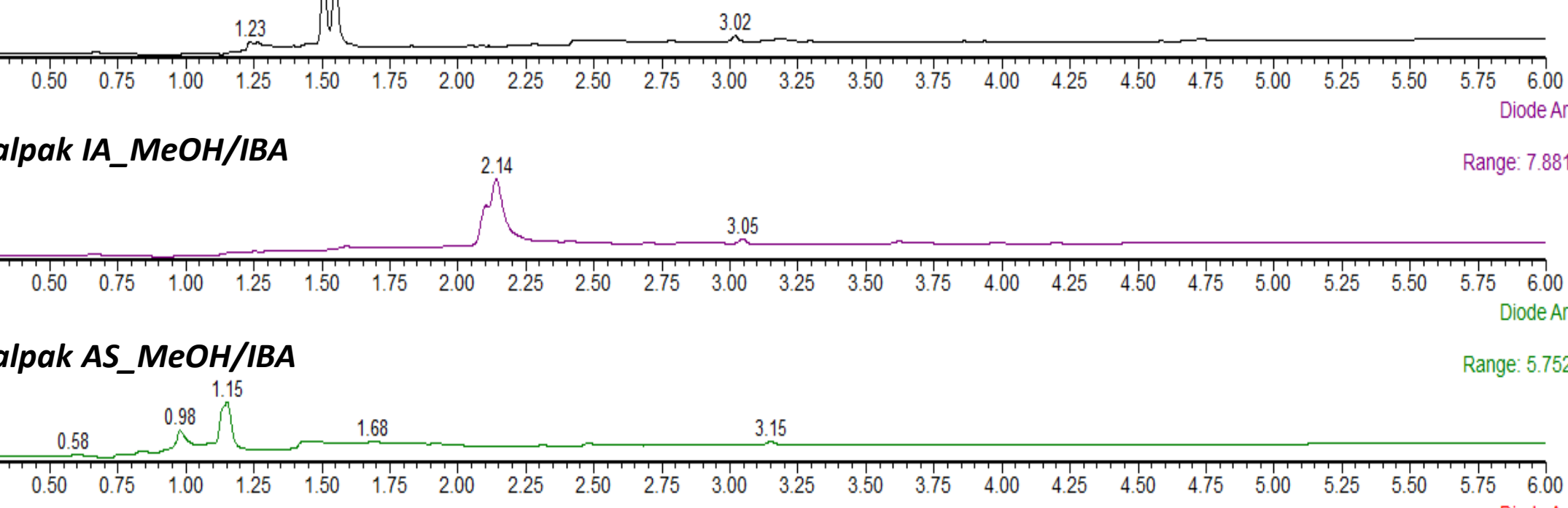

Range: 1.054 2014-01-30_Leo-010

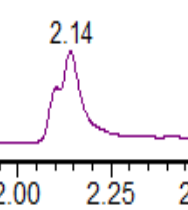

Range 78810
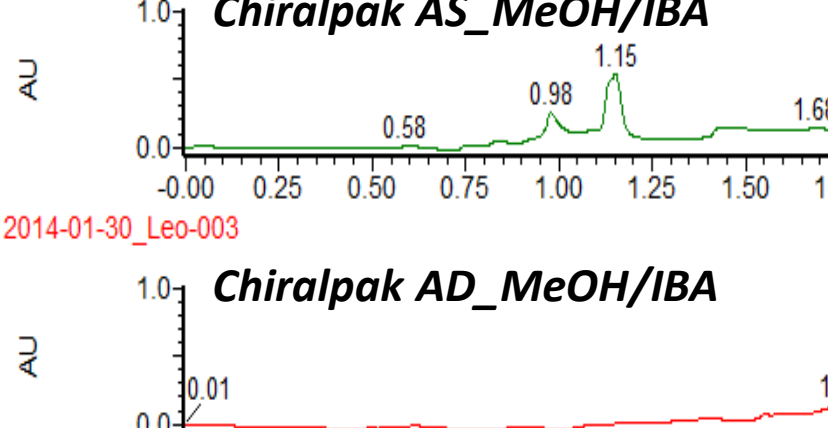

$\begin{array}{llllllllllll}1.25 & 1.25 & 1.75 & 2.00 & 2.25 & 250 & 2.75\end{array}$ 
SFC Screening: 1-40\% (0-5 min), 40\% (5-6 min), no post. $3 \mathrm{~mL} / \mathrm{min}, 200 \mathrm{bar}, 40^{\circ} \mathrm{C}$

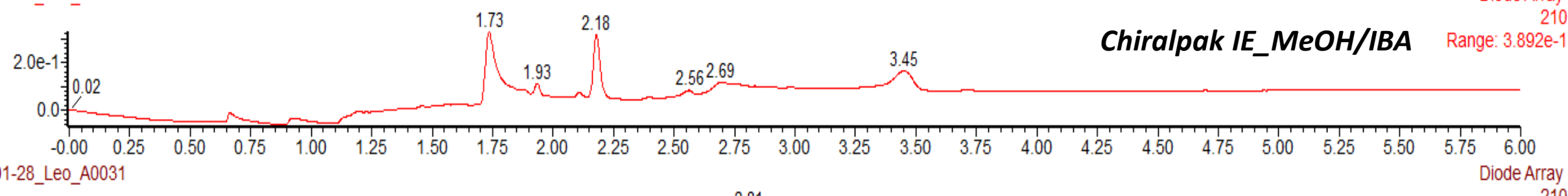
2014-01-28_Leo_A0031
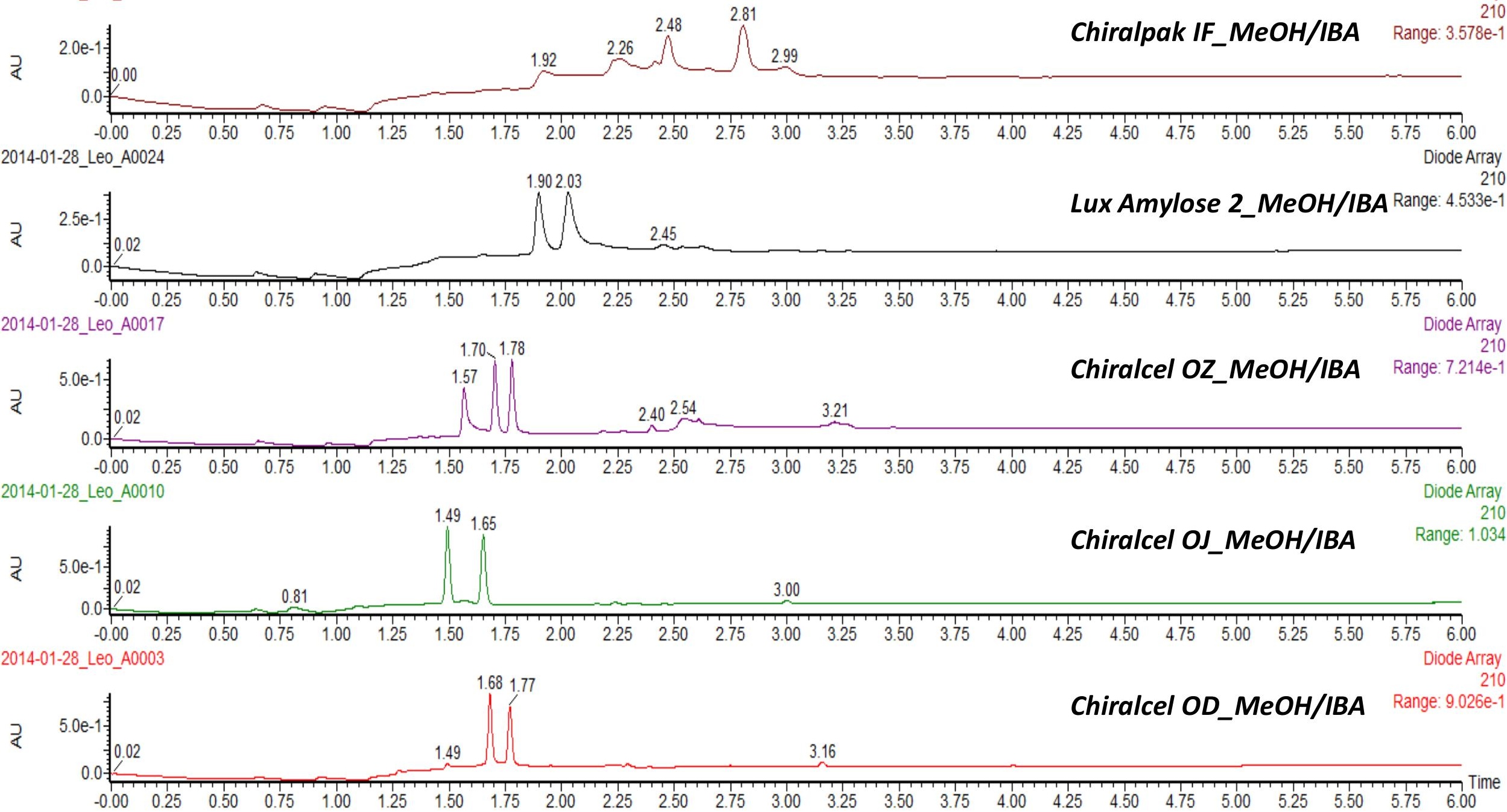
SFC Screening: 1-40\% (0-5 min), 40\% (5-6 min), no post. $3 \mathrm{~mL} / \mathrm{min}, 200 \mathrm{bar}, 40^{\circ} \mathrm{C}$

SFC Screening: 1-40\% (0-5 min), 40\% (5-6 min), no post. $3 \mathrm{~mL} / \mathrm{min}, 200 \mathrm{bar}, 40^{\circ} \mathrm{C}$
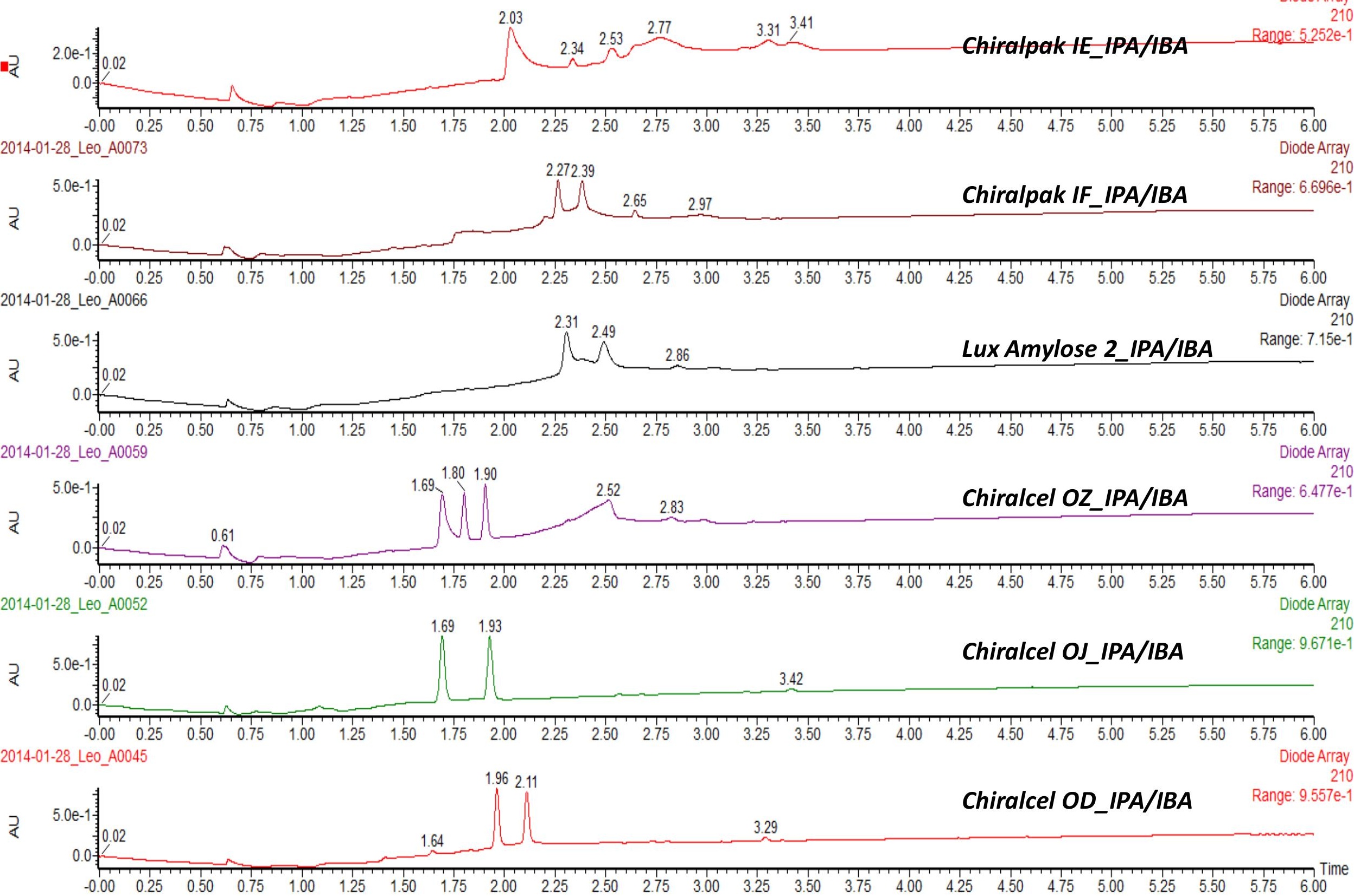
SFC Screening: $1-40 \%(0-5 \mathrm{~min}), 40 \%(5-6 \mathrm{~min})$, no post. $3 \mathrm{~mL} / \mathrm{min}, 200 \mathrm{bar}, 40^{\circ} \mathrm{C}$

S28
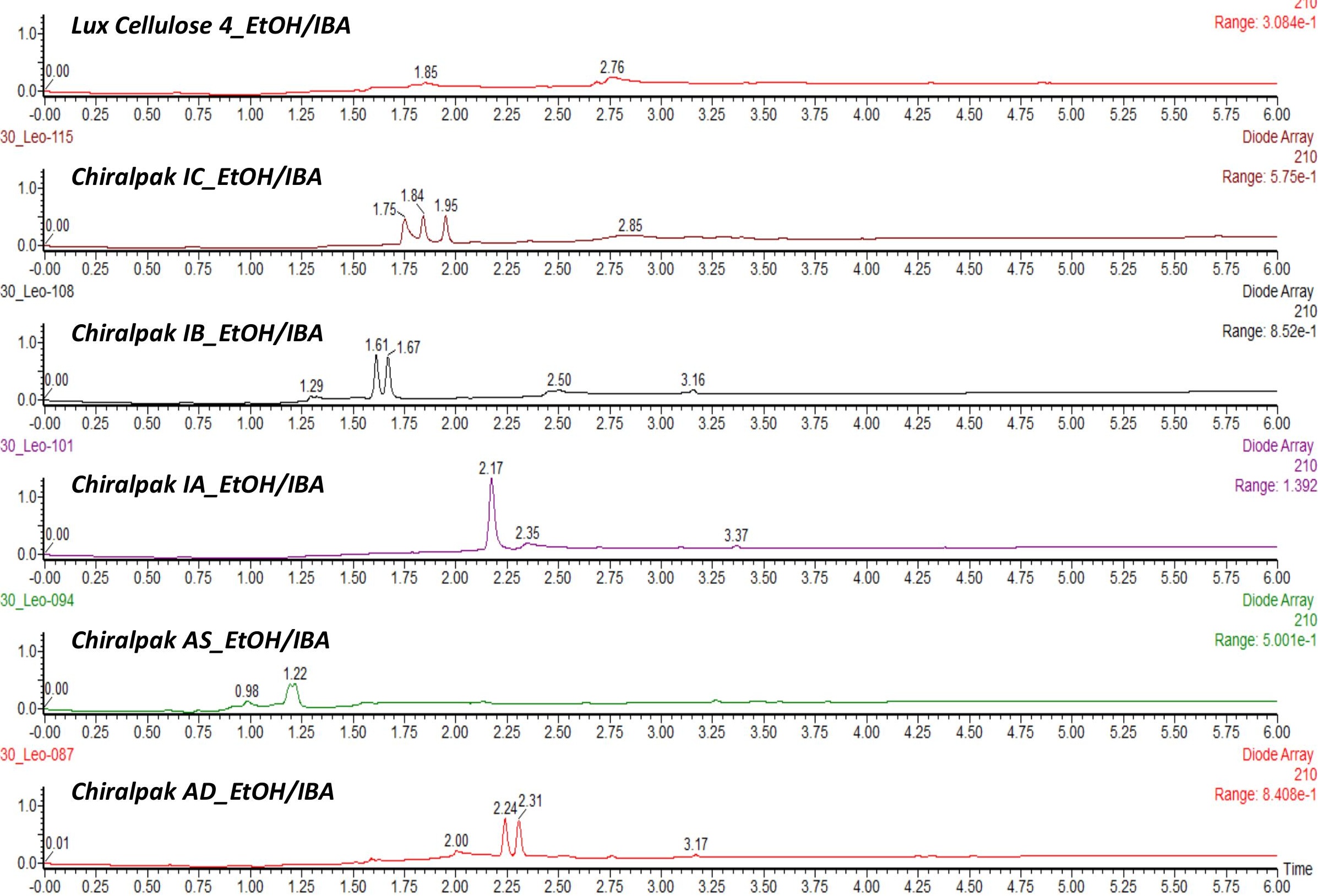
SFC Screening: 1-40\% (0-5 $\mathrm{min}), 40 \%(5-6 \mathrm{~min})$, no post. $3 \mathrm{~mL} / \mathrm{min}, 200 \mathrm{bar}, 40^{\circ} \mathrm{C}$
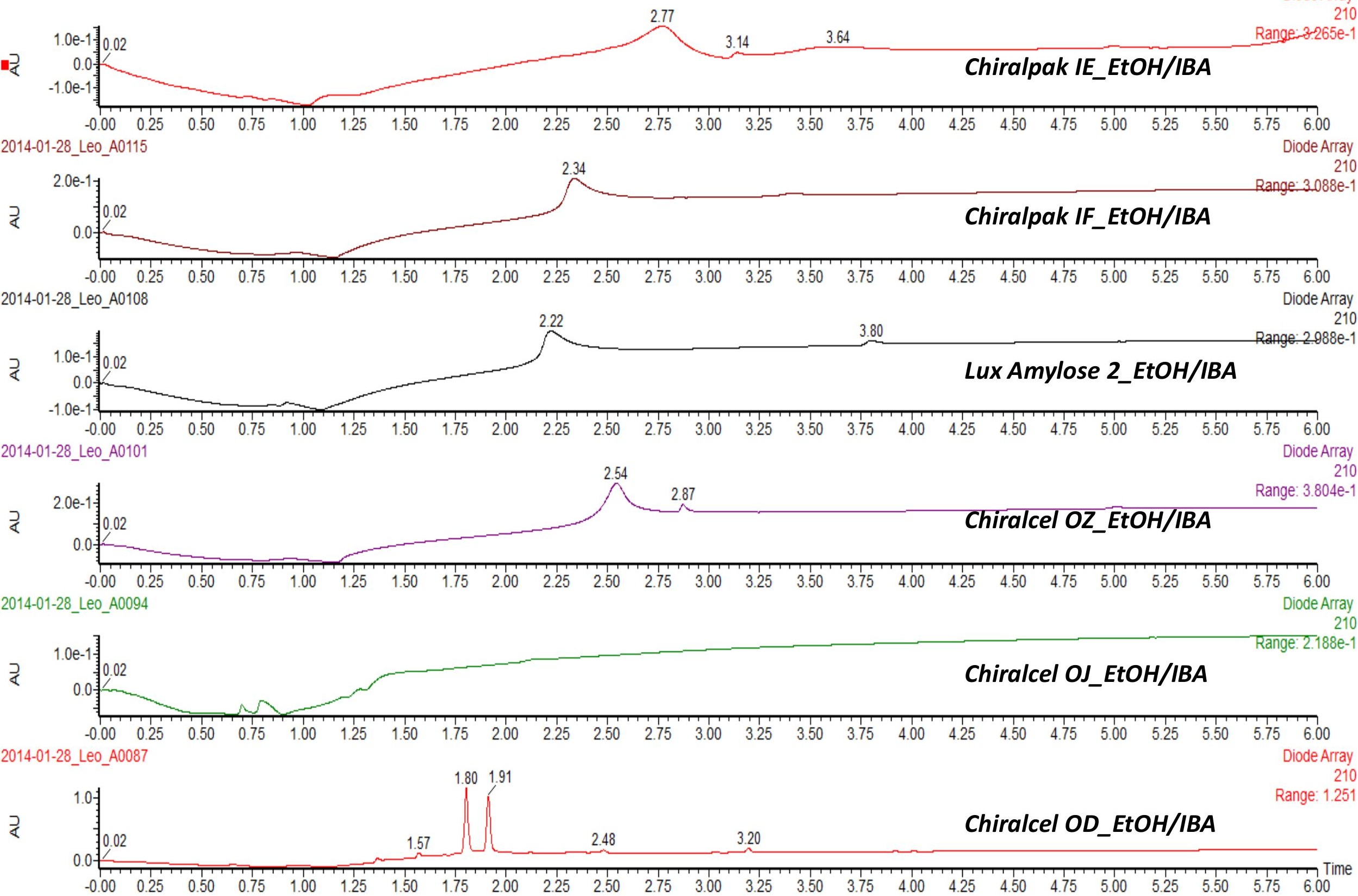

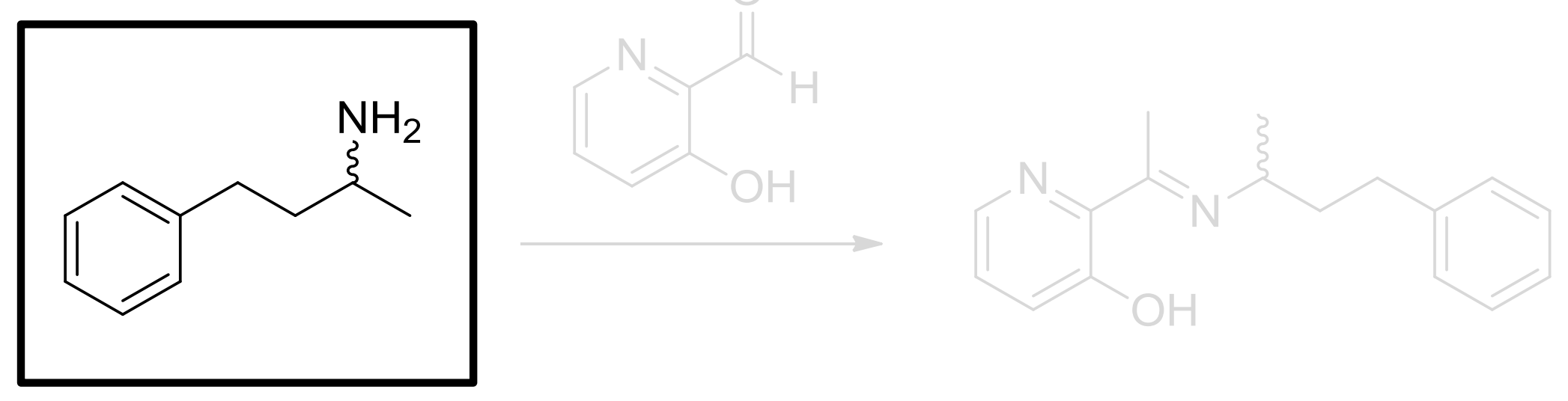

Primary Chiral SFC Screen

12 columns, 3 solvents

(with and without 25 mM IBA) 
SFC Screening: $1-40 \%(0-5 \mathrm{~min}), 40 \%(5-6 \mathrm{~min})$, no post. $3 \mathrm{~mL} / \mathrm{min}, 200 \mathrm{bar}, 40^{\circ} \mathrm{C}$

S31
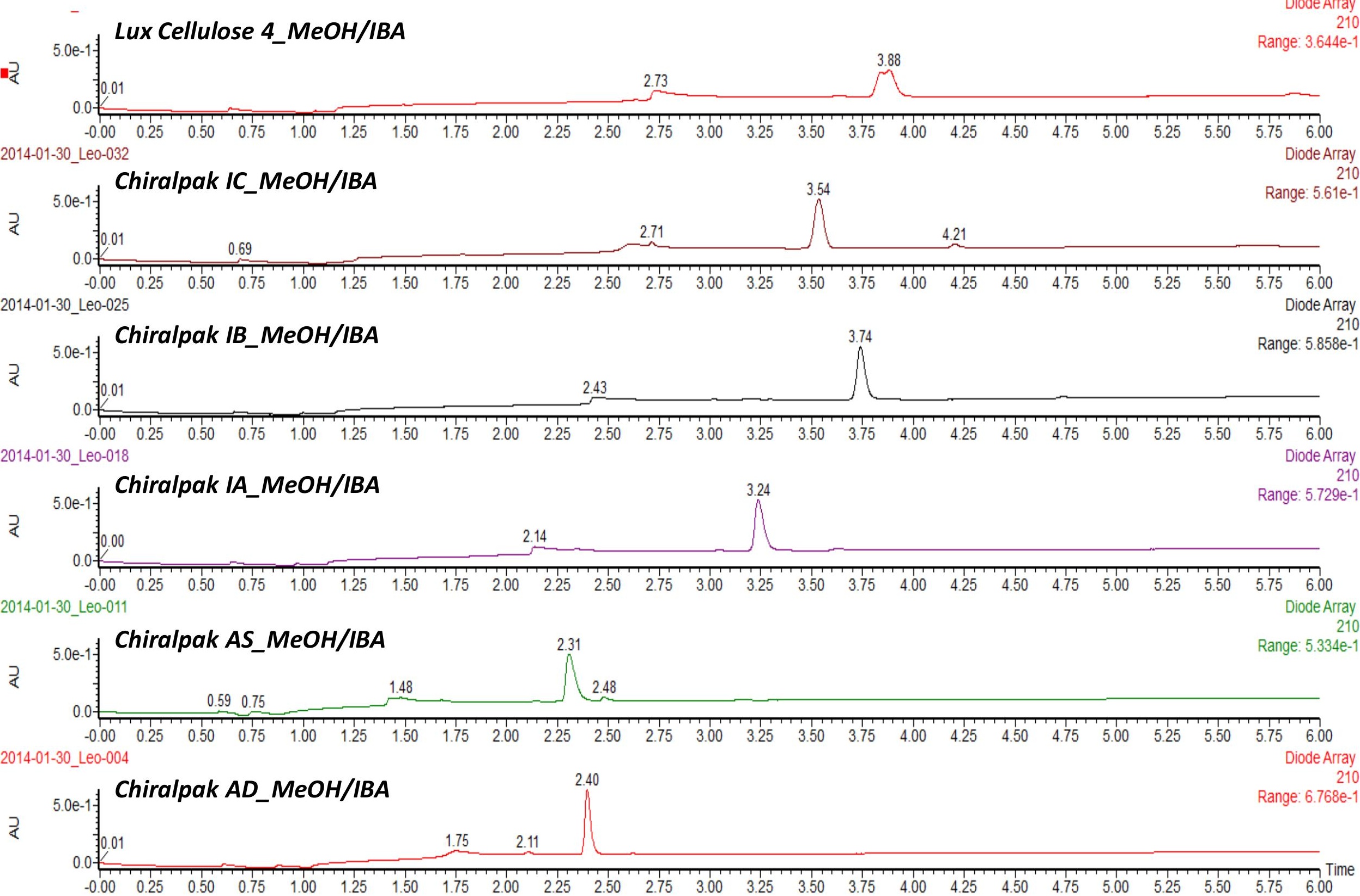
SFC Screening: 1-40\% (0-5 min), 40\% (5-6 min), no post. $3 \mathrm{~mL} / \mathrm{min}, 200 \mathrm{bar}, 40^{\circ} \mathrm{C}$

\section{S32}

MPP Amine_2

Diode Array
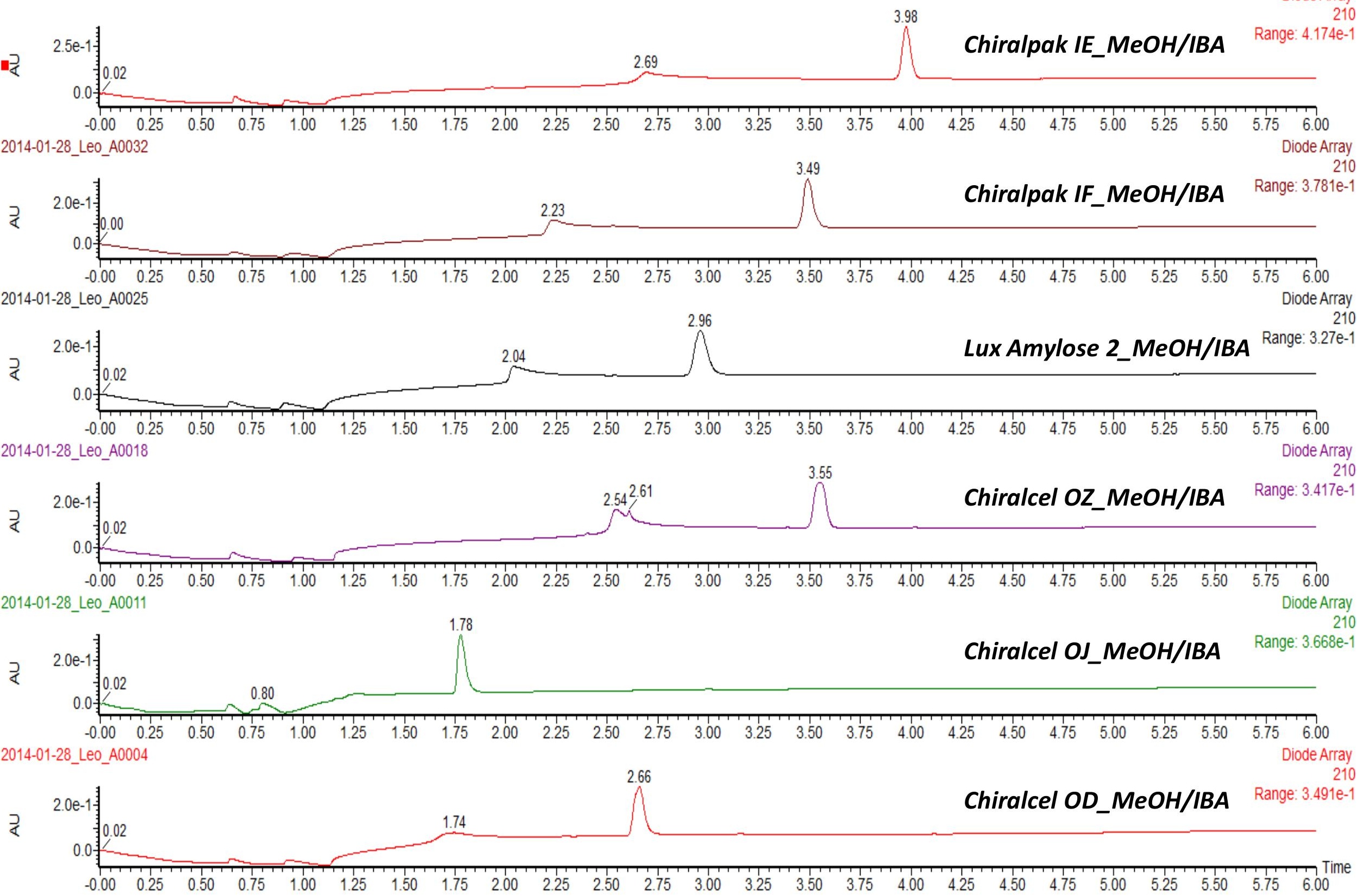


\section{SFC Screening: 1-40\% (0-5 min), 40\% (5-6 min), no post. $3 \mathrm{~mL} / \mathrm{min}, 200 \mathrm{bar}, 40^{\circ} \mathrm{C}$}

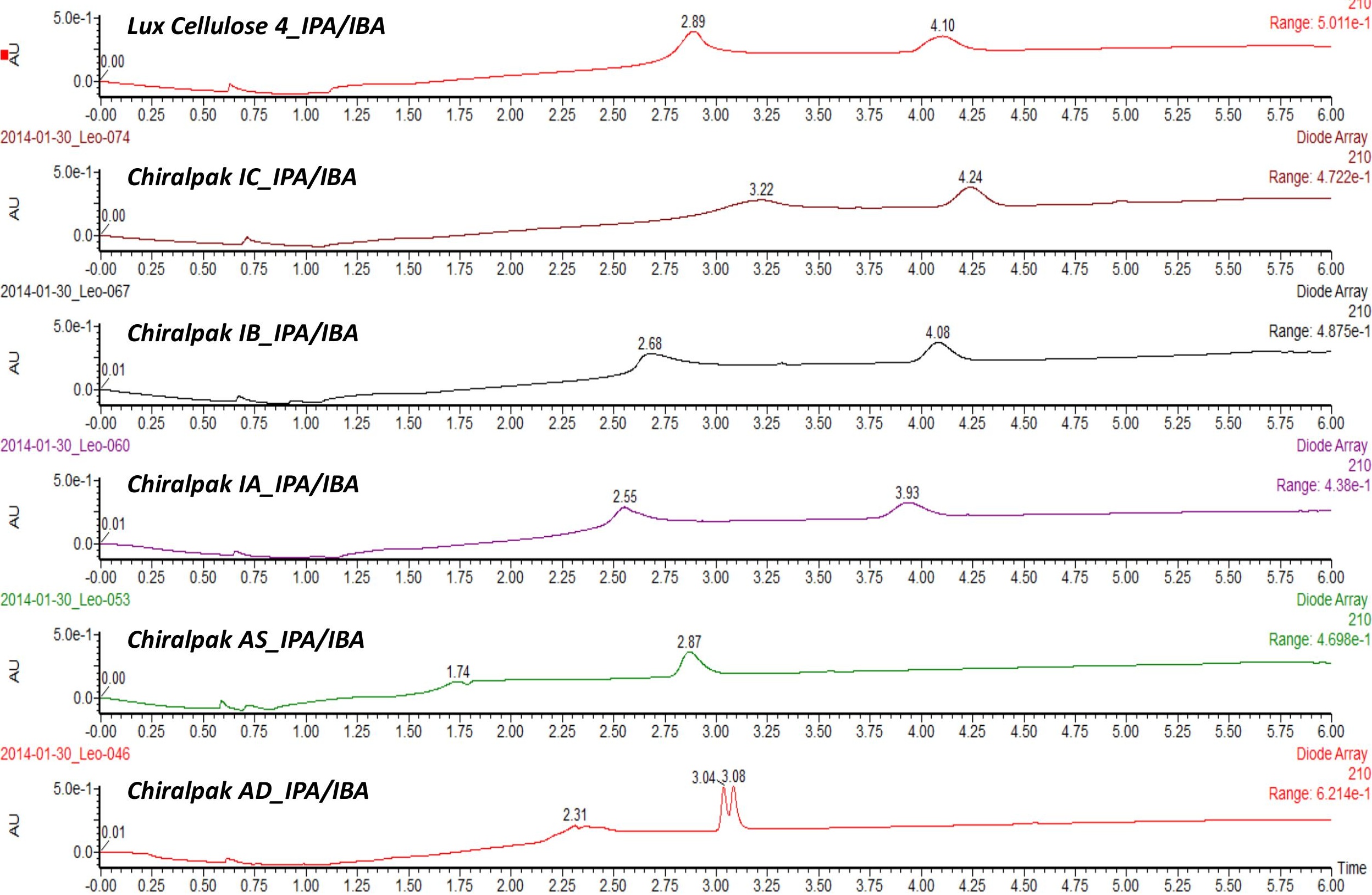


SFC Screening: 1-40\% (0-5 min), 40\% (5-6 min), no post. $3 \mathrm{~mL} / \mathrm{min}, 200 \mathrm{bar}, 40^{\circ} \mathrm{C}$

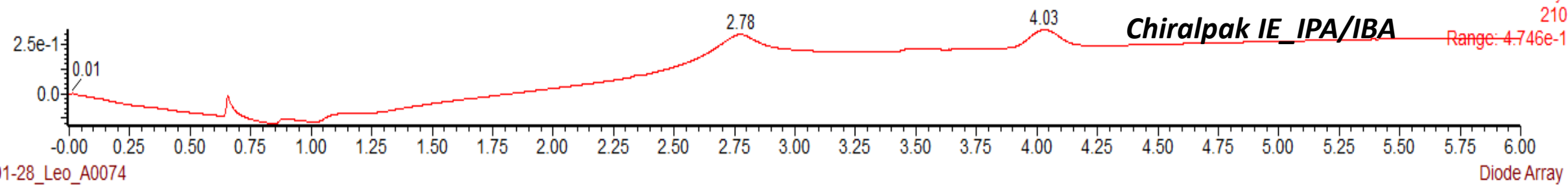
014-01-28_Leo A0074
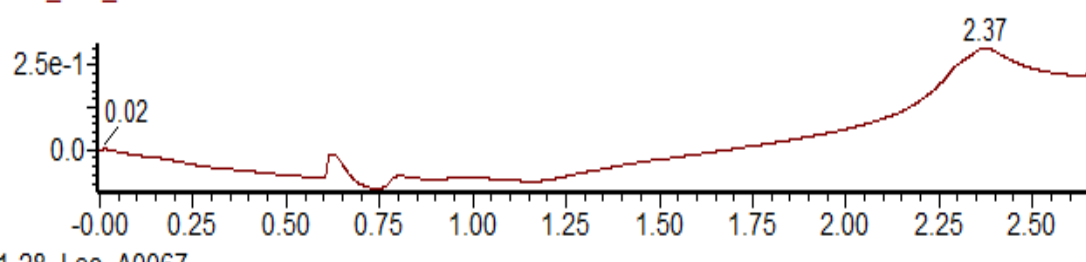

Chiralpak IF_IPA/IBA

iode Array 2014-01-28_Leo_A0067
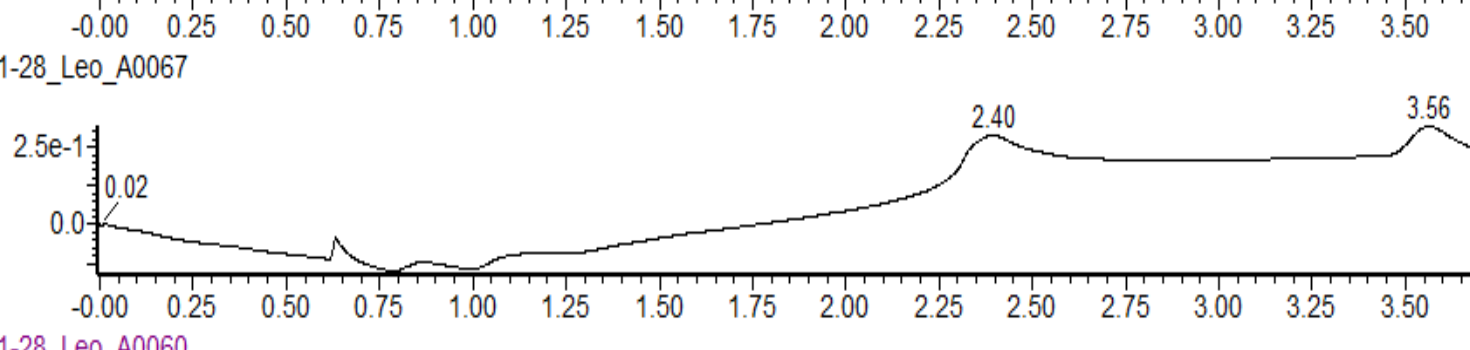

$$
2.0 \mathrm{e}-1
$$
eo A0060
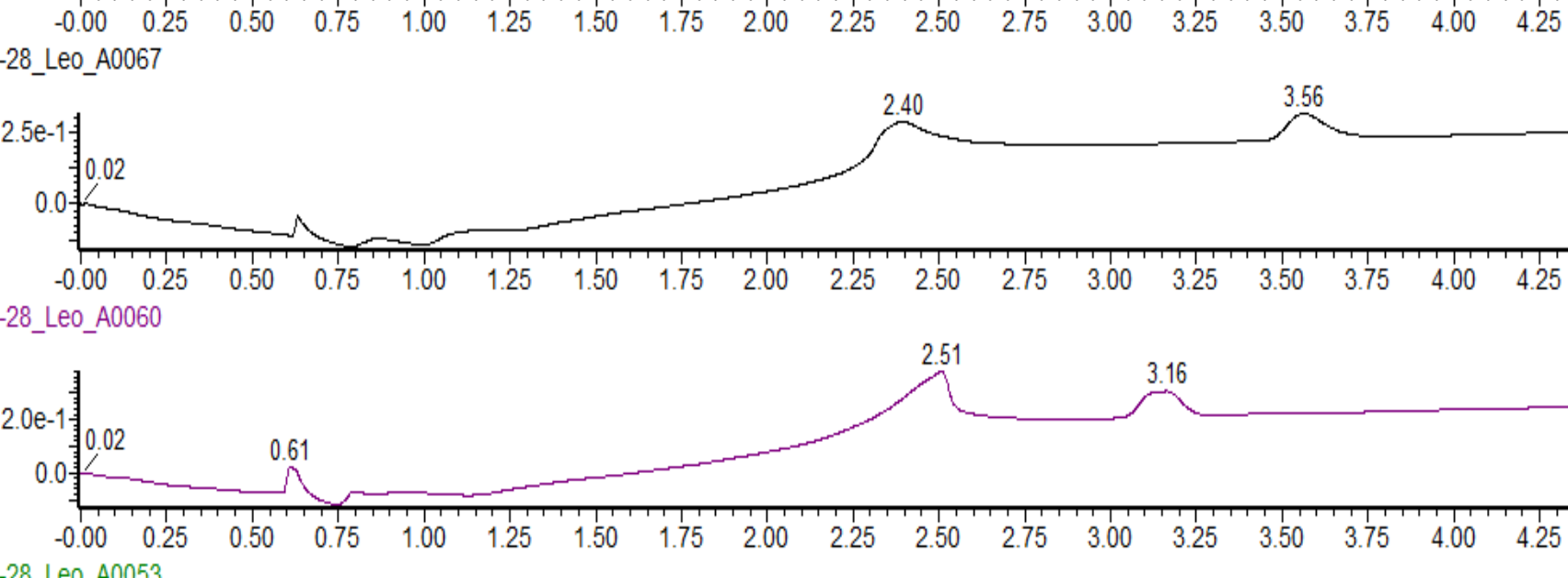

Lux Amylose 2_IPA/IBA

5.00

2014-01-28_LeO_A0053

?
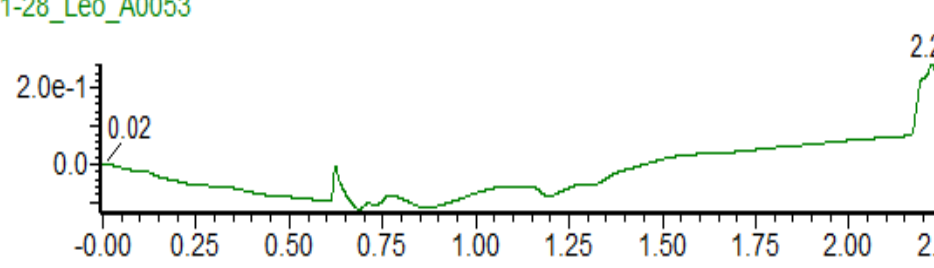

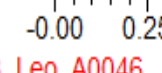

2014
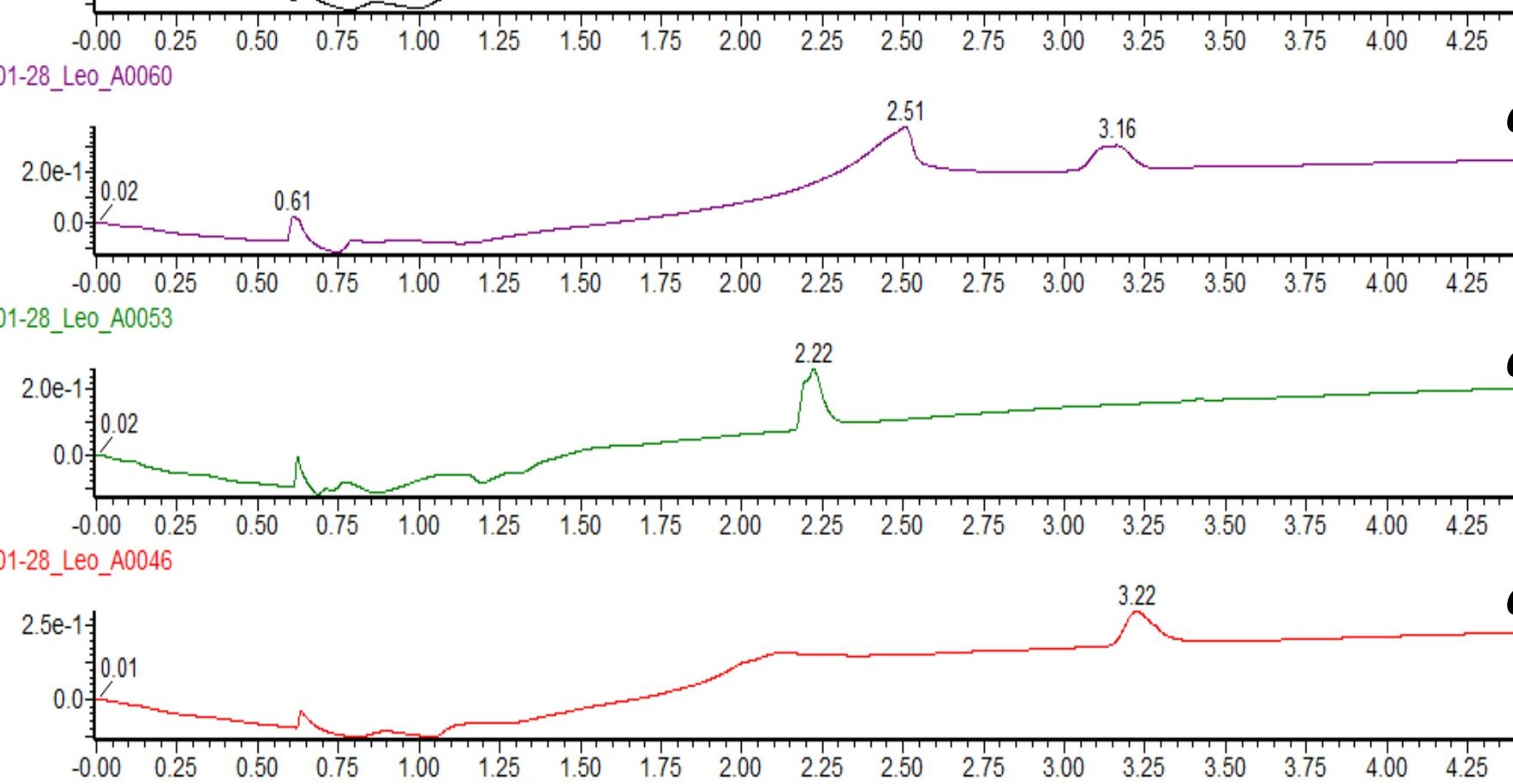

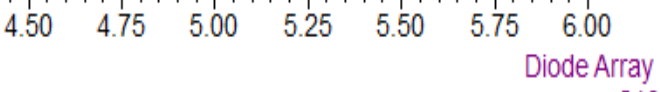

Chiralcel OZ_IPA/IBA Range: $5.0 \mathrm{e}-1$

210 
SFC Screening: $1-40 \%(0-5 \mathrm{~min}), 40 \%(5-6 \mathrm{~min})$, no post. $3 \mathrm{~mL} / \mathrm{min}, 200 \mathrm{bar}, 40^{\circ} \mathrm{C}$

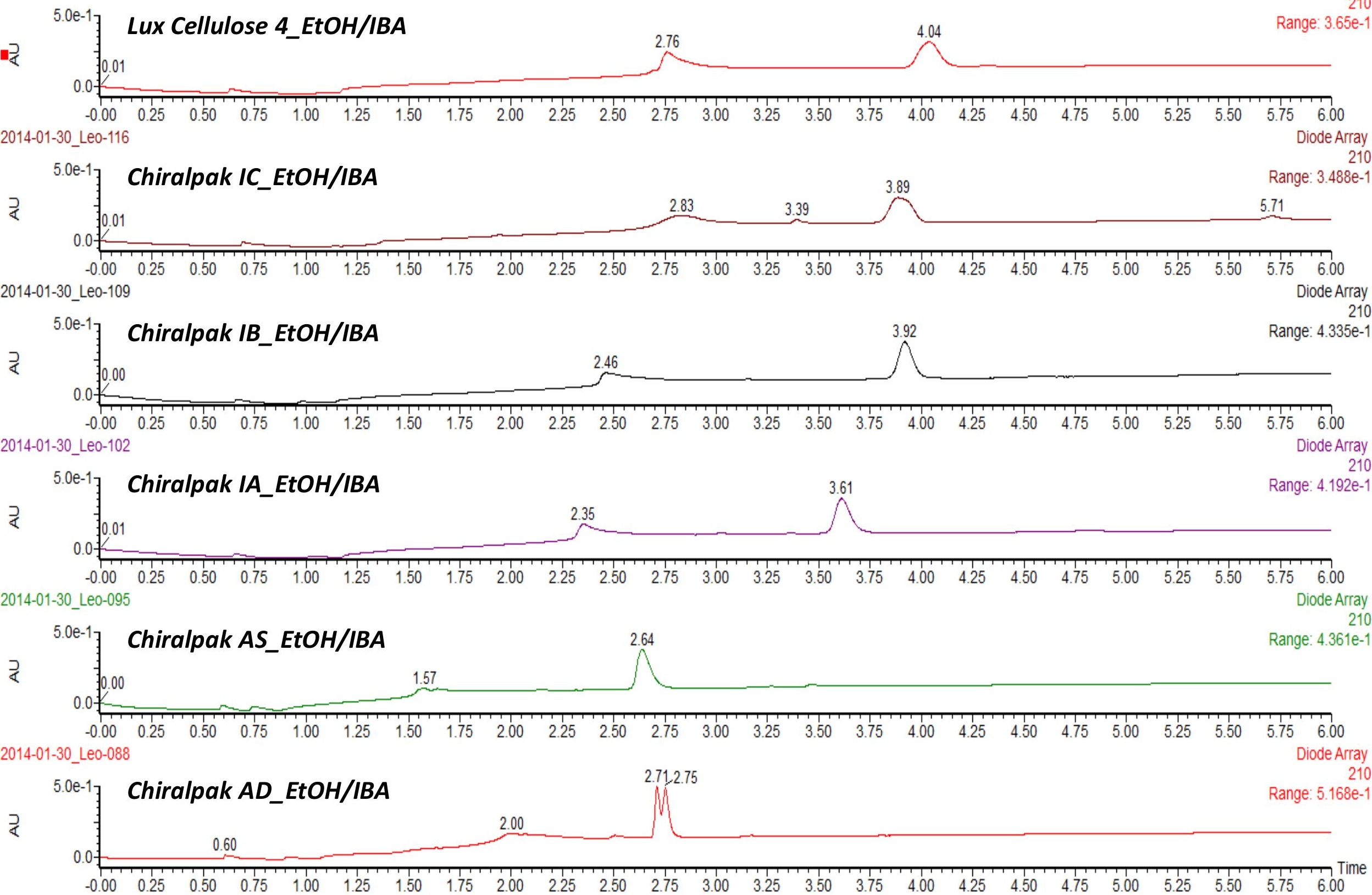


SFC Screening: 1-40\% (0-5 min), 40\% (5-6 min), no post. $3 \mathrm{~mL} / \mathrm{min}, 200 \mathrm{bar}, 40^{\circ} \mathrm{C}$

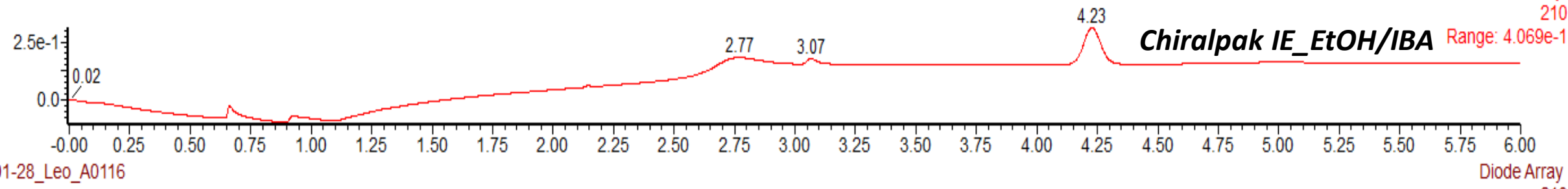
24-01-28 Leo A011

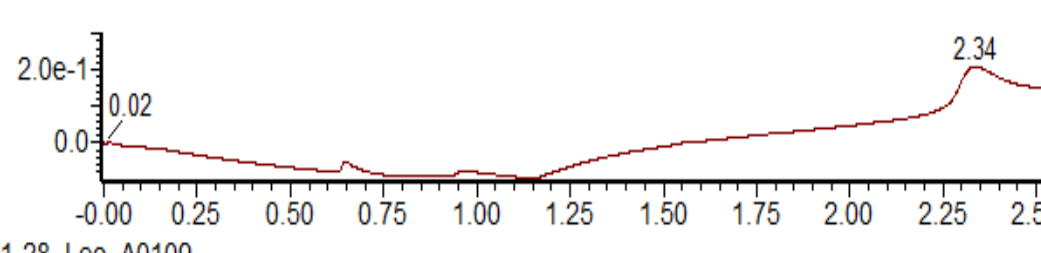

Chiralpak IF_EtOH/IBA iode Array 2014-01-28_Leo_A0109
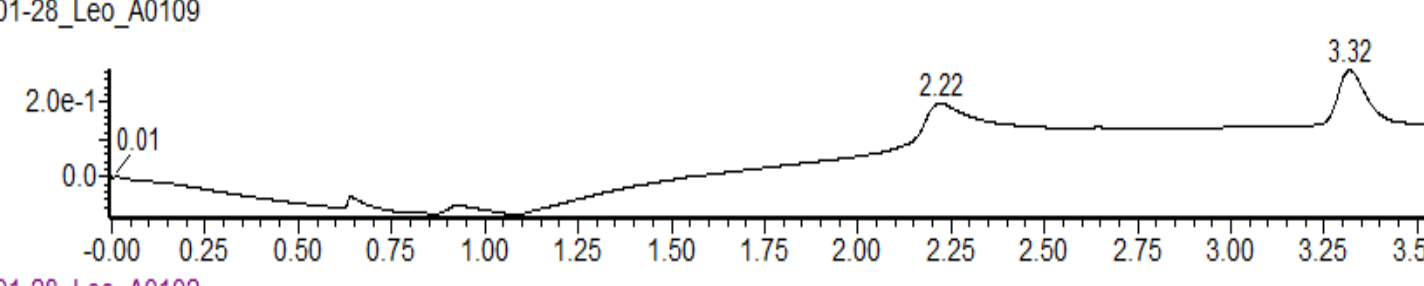

$\frac{2}{\varangle}$

$\begin{array}{rr}-0.00 & 0.25 \\ & \end{array}$

2.0e-1
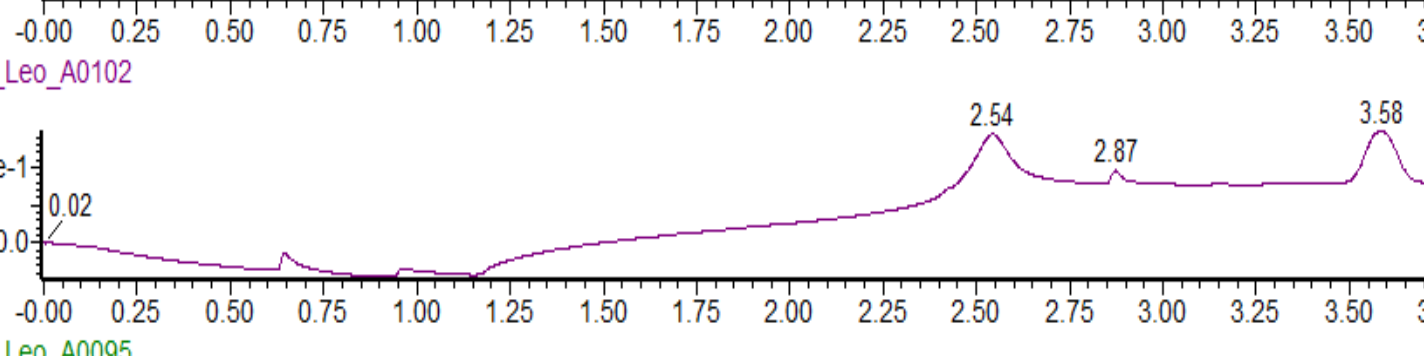

2014-01-28_Leo_A0095
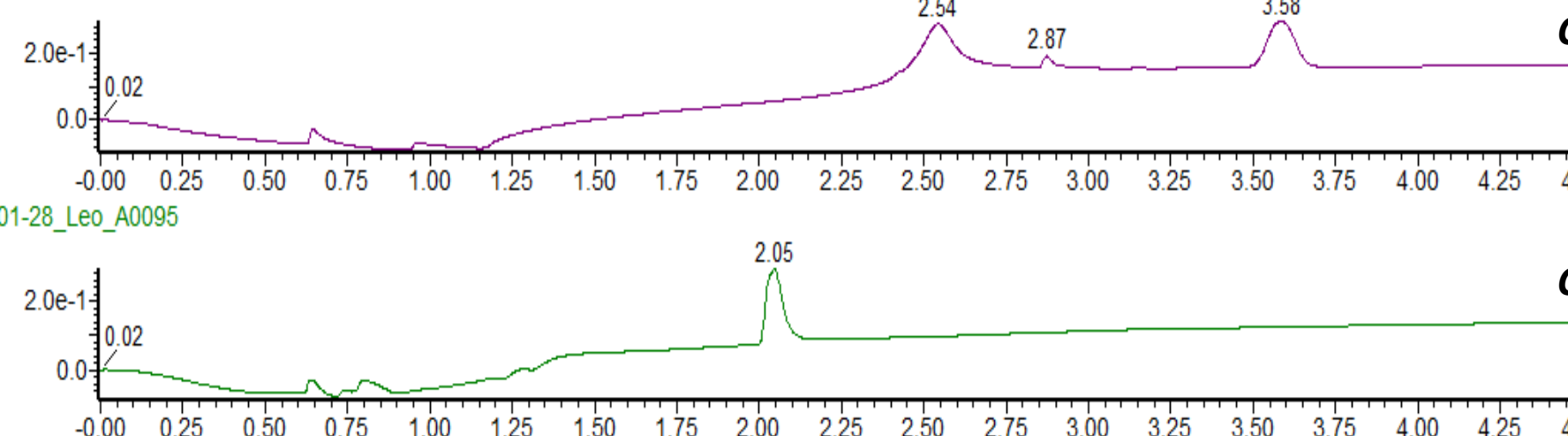

3.80

Lux Amylose 2_EtOH/IBAange

6.00

210

210
$7 e-1$ 2014-01-28_Leo_A0088

$\frac{2}{\&}$
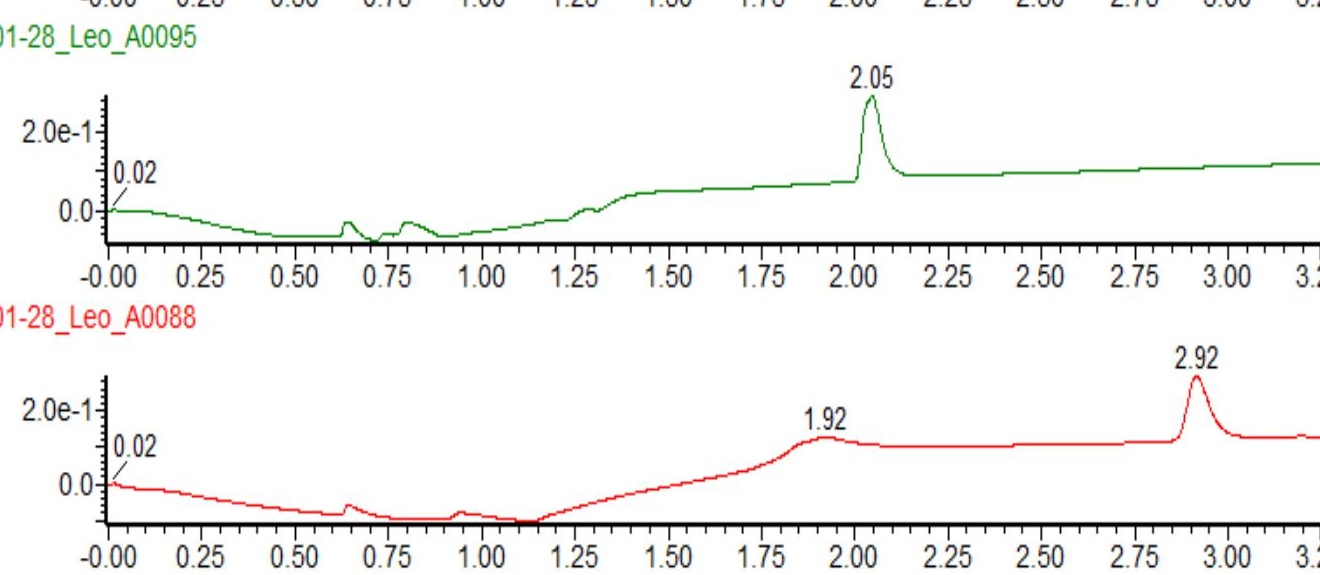


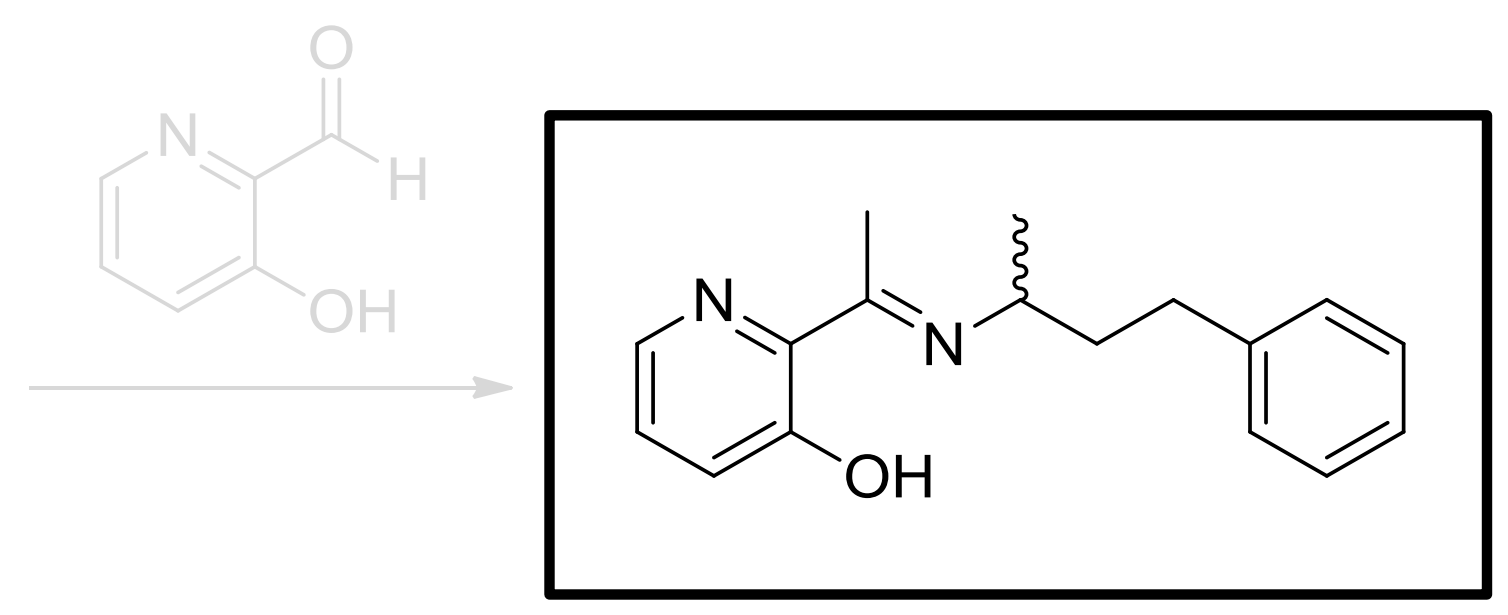

Primary Chiral SFC Screen

12 columns, 3 solvents

(with and without $25 \mathrm{mM}$ IBA) 
SFC Screening: $1-40 \%(0-5 \mathrm{~min}), 40 \%(5-6 \mathrm{~min})$, no post. $3 \mathrm{~mL} / \mathrm{min}, 200 \mathrm{bar}, 40^{\circ} \mathrm{C}$

S38
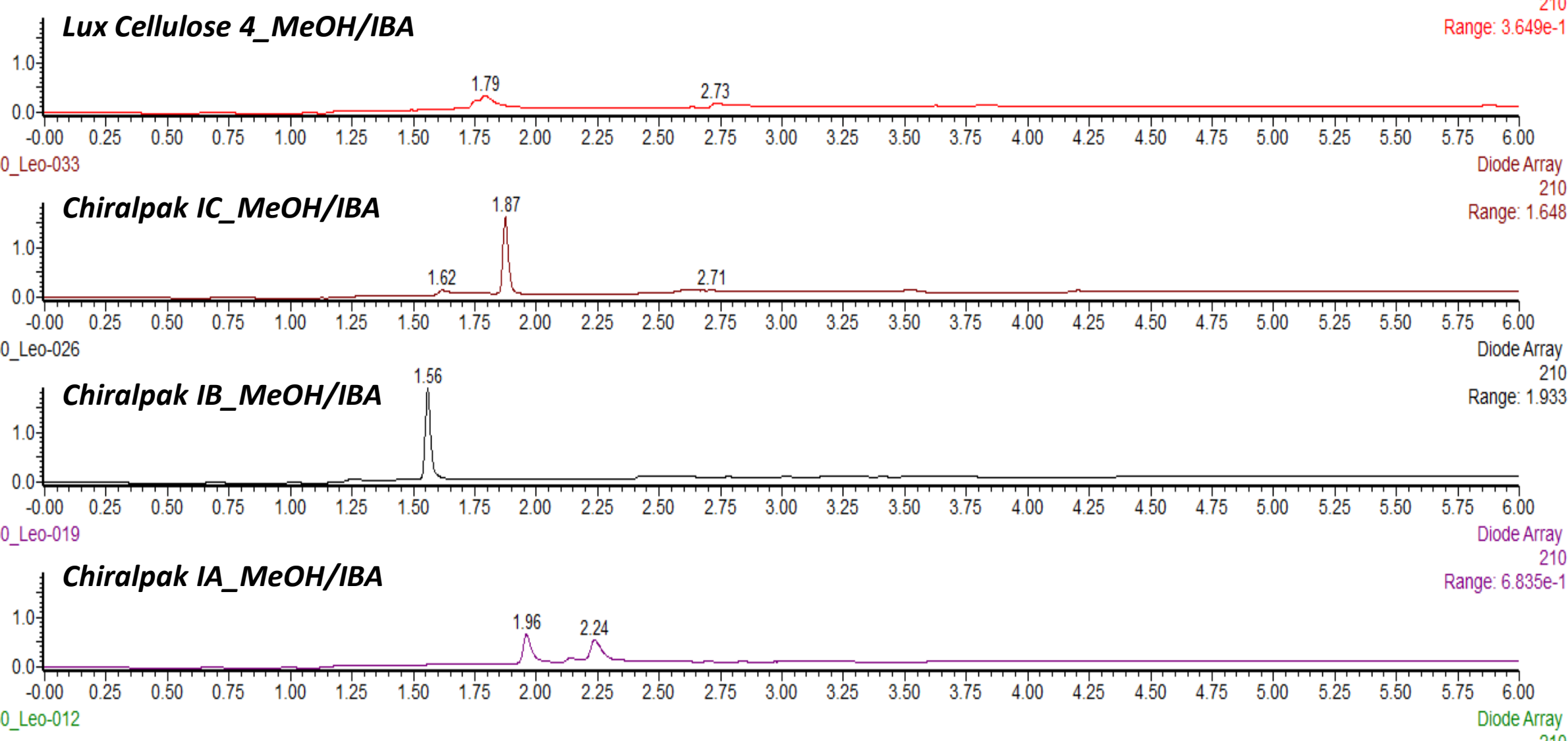

\section{: Chiralpak AS_MeOH/IBA}

Range: 1.355

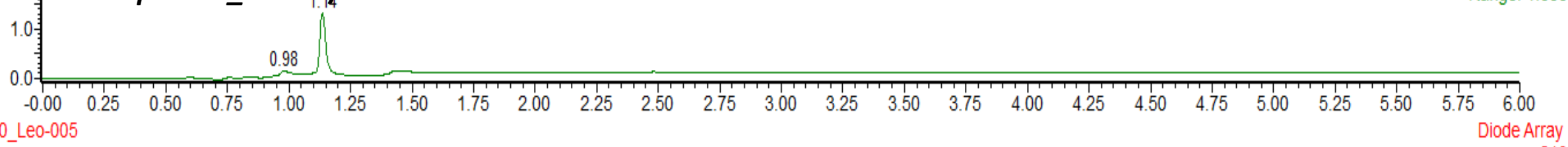

: Chiralpak AD_MeOH/IBA

Range: $9.594 \mathrm{e}-1$ 
SFC Screening: 1-40\% (0-5 min), 40\% (5-6 min), no post. $3 \mathrm{~mL} / \mathrm{min}, 200 \mathrm{bar}, 40^{\circ} \mathrm{C}$

SFC Screening: $1-40 \%(0-5 \mathrm{~min}), 40 \%(5-6 \mathrm{~min})$, no post. $3 \mathrm{~mL} / \mathrm{min}, 200 \mathrm{bar}, 40^{\circ} \mathrm{C}$
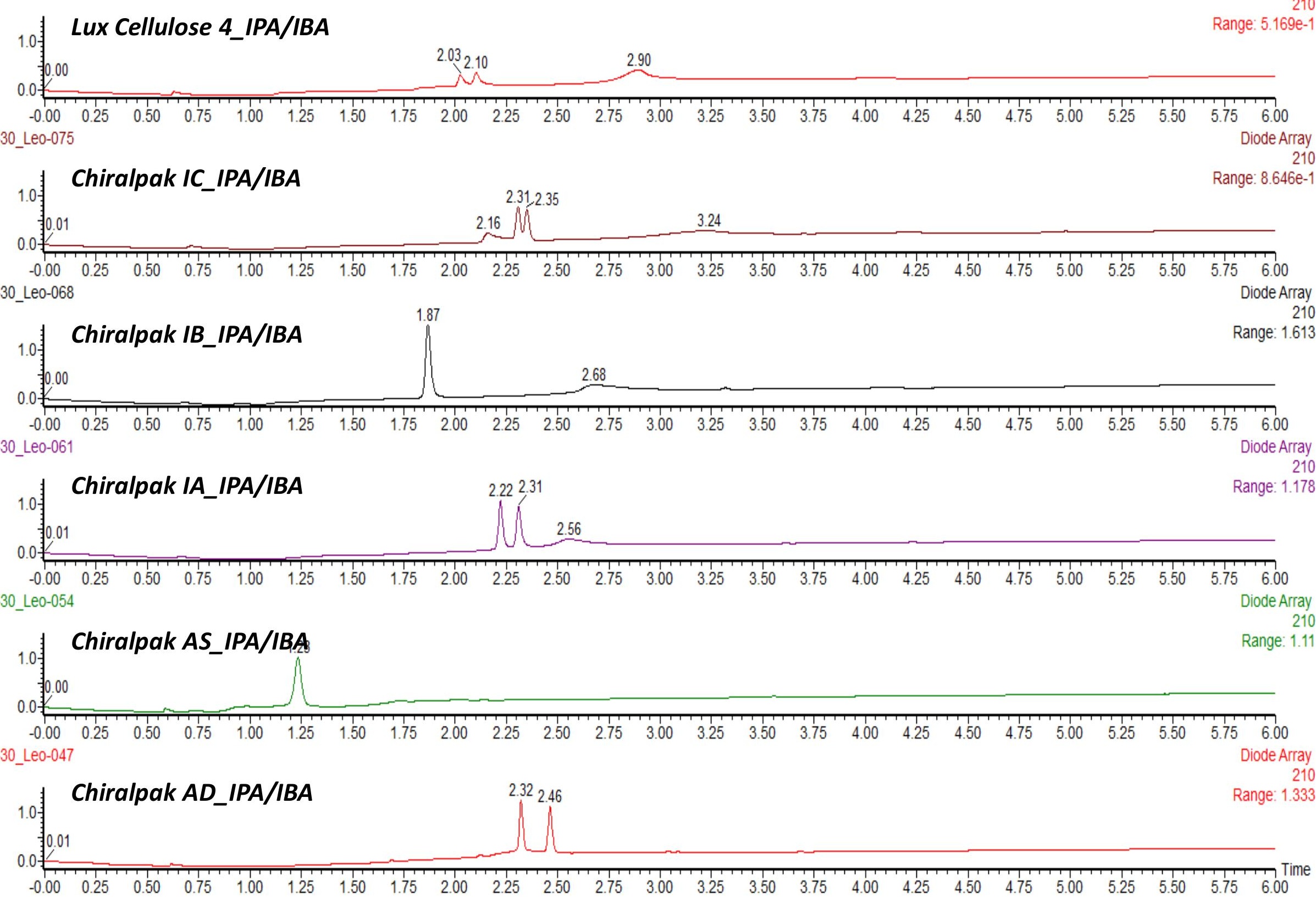
SFC Screening: $1-40 \%(0-5 \mathrm{~min}), 40 \%(5-6 \mathrm{~min})$, no post. $3 \mathrm{~mL} / \mathrm{min}, 200 \mathrm{bar}, 40^{\circ} \mathrm{C}$

S41

MPP Imine_2
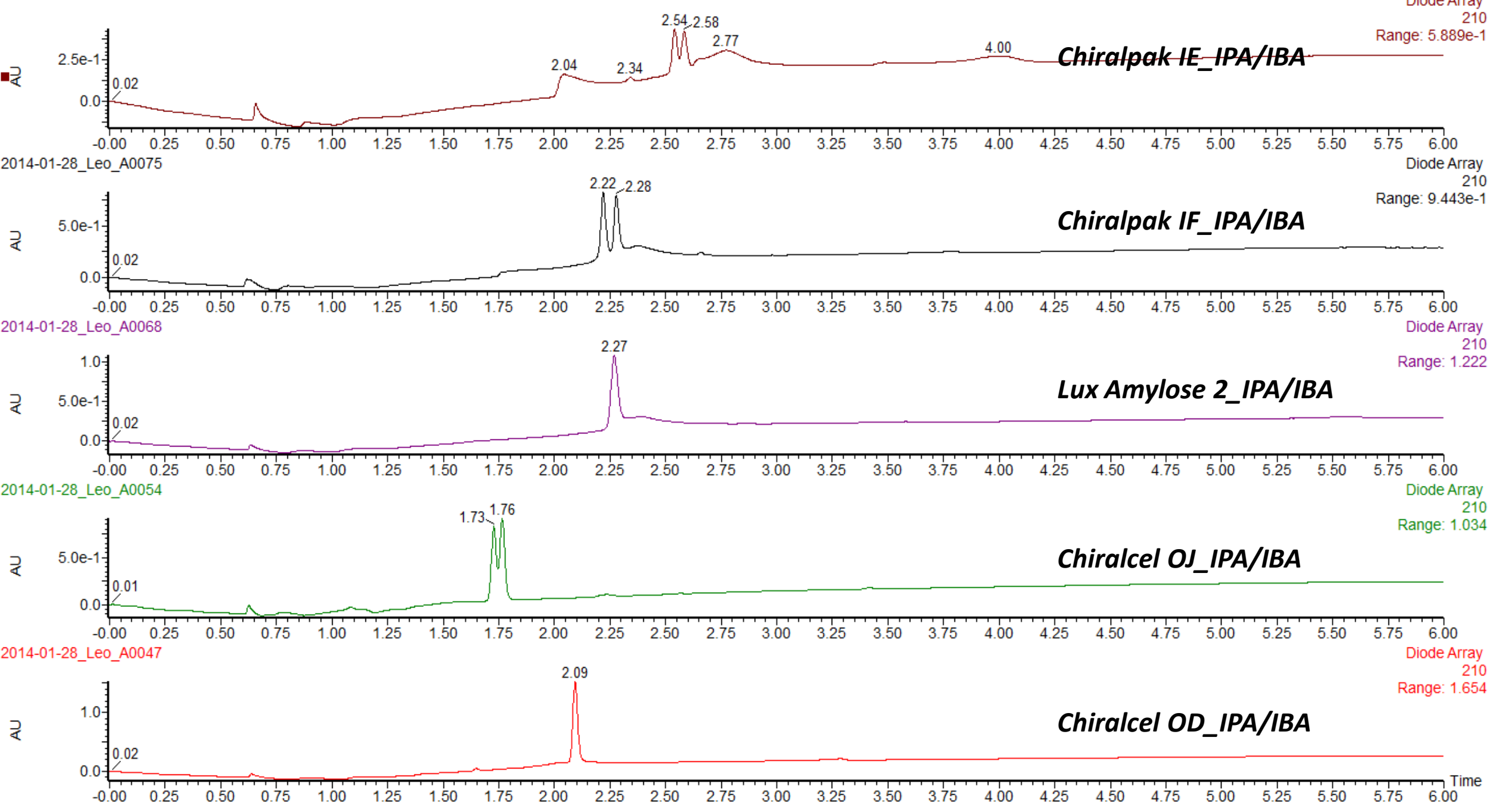
SFC Screening: $1-40 \%(0-5 \mathrm{~min}), 40 \%(5-6 \mathrm{~min})$, no post. $3 \mathrm{~mL} / \mathrm{min}, 200 \mathrm{bar}, 40^{\circ} \mathrm{C}$

S42

\section{Lux Cellulose 4_EtOH/IBA}

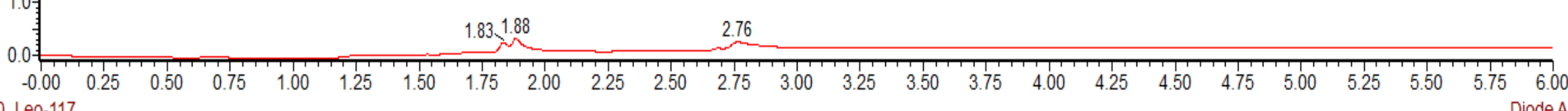

2014-01-30_Leo-117

\section{: Chiralpak IC_EtOH/IBA}

Diode Array

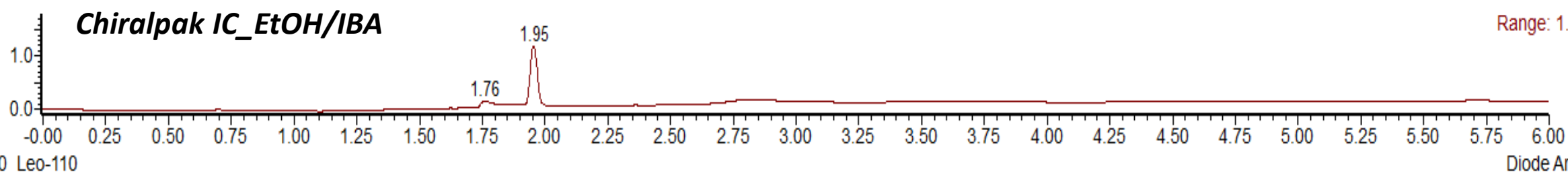
2014-01-30_Leo-110

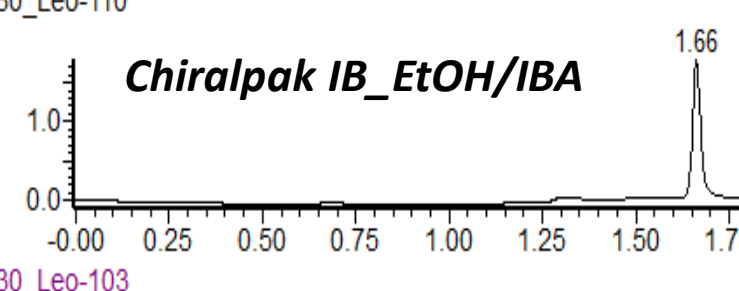

2014-01-30_Leo-103
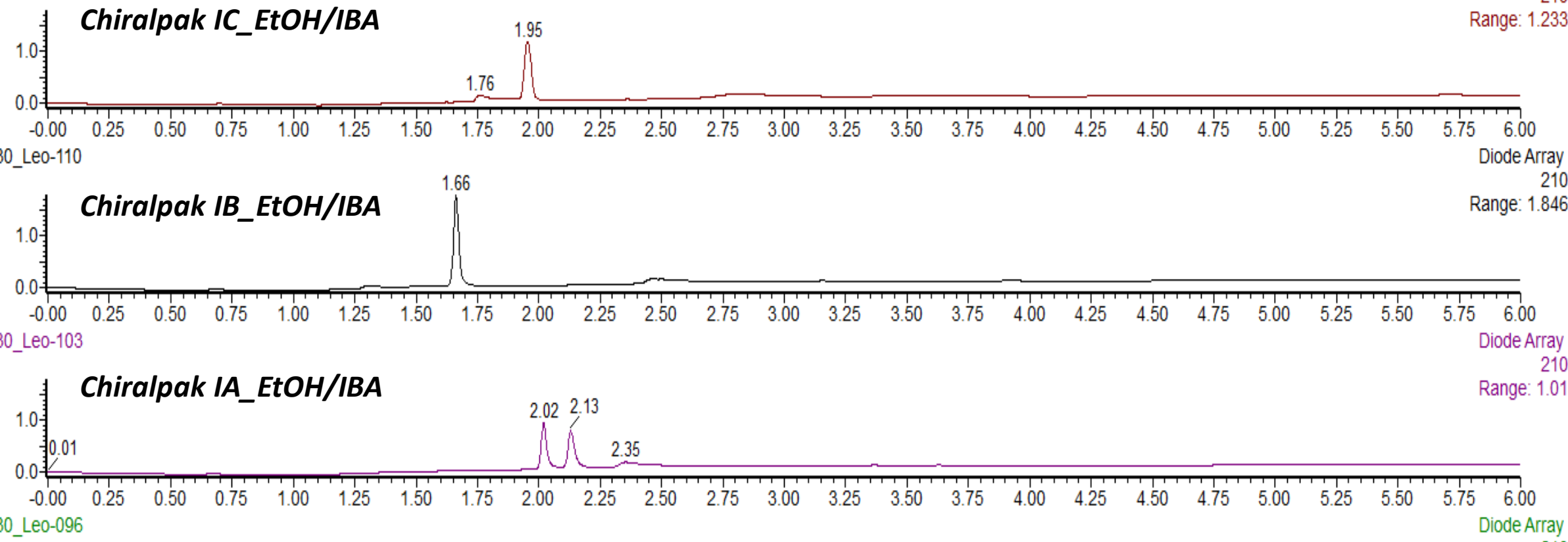

\section{Chiralpak AS_EtOH/IBAA}

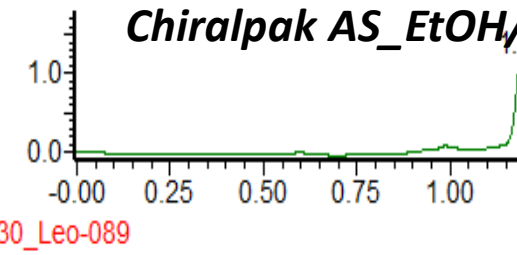

$$
0.0 \frac{\text { the }}{-0.00}
$$

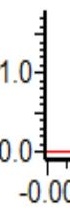

Chiralpak AD_EtOH/IBA

\begin{tabular}{llllllllllllll} 
& \\
\hline
\end{tabular}


SFC Screening: 1-40\% (0-5 min), 40\% (5-6 min), no post. $3 \mathrm{~mL} / \mathrm{min}, 200 \mathrm{bar}, 40^{\circ} \mathrm{C}$

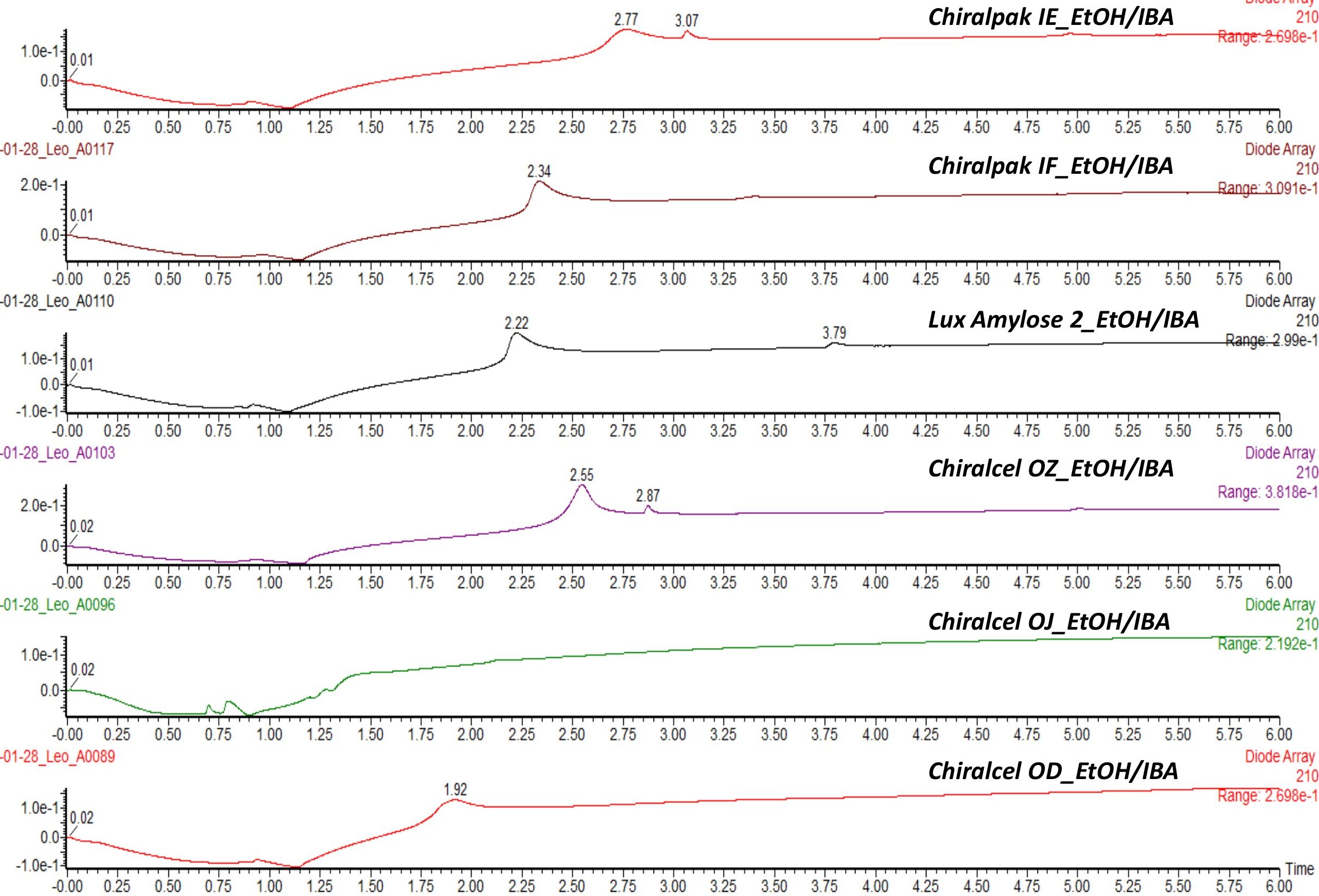




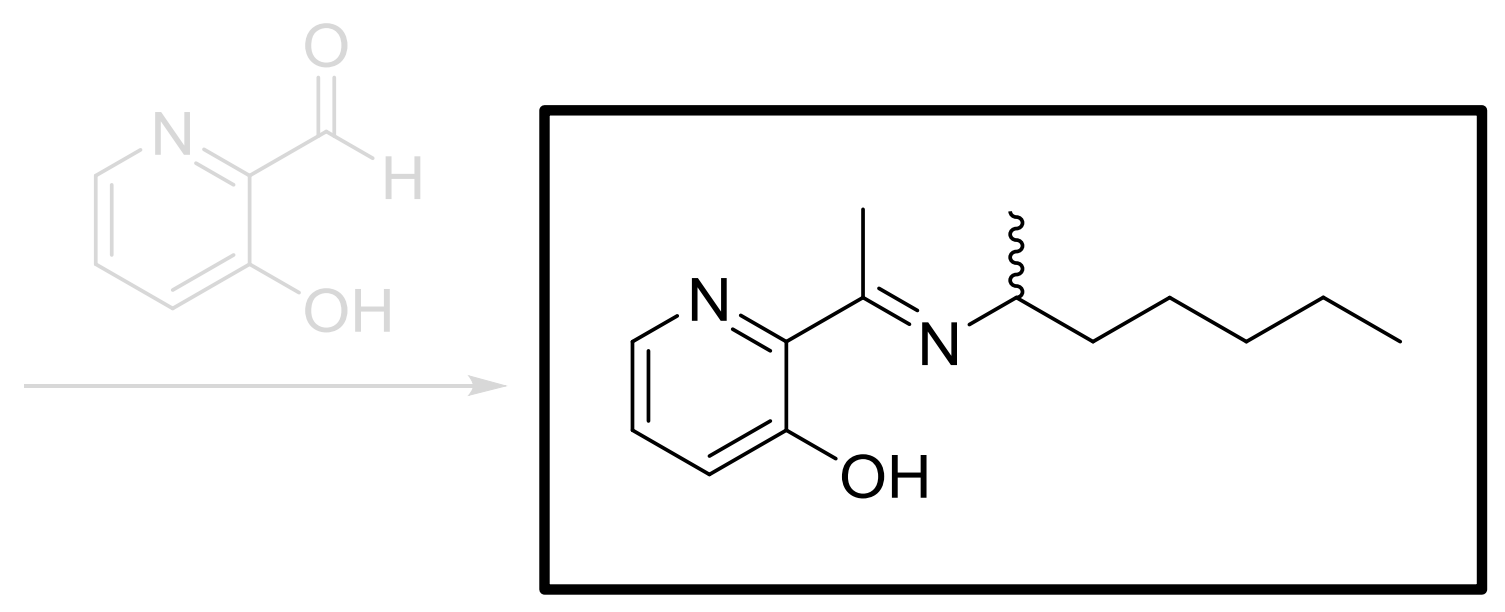

Primary Chiral SFC Screen

12 columns, 3 solvents

(with and without 25 mM IBA) 
SFC Screening: $1-40 \%(0-5 \mathrm{~min}), 40 \%(5-6 \mathrm{~min})$, no post. $3 \mathrm{~mL} / \mathrm{min}, 200 \mathrm{bar}, 40^{\circ} \mathrm{C}$

1.0 Lux Cellulose 4_MeOH/IBA

Range: $2963 \mathrm{e}-1$

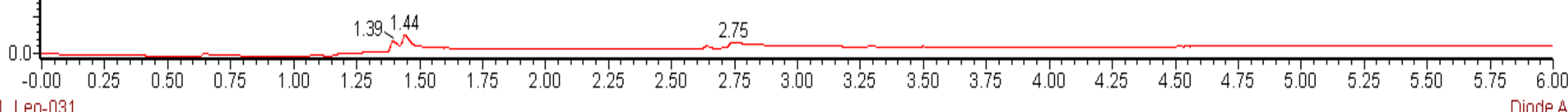

2014-02-11_Leo-03

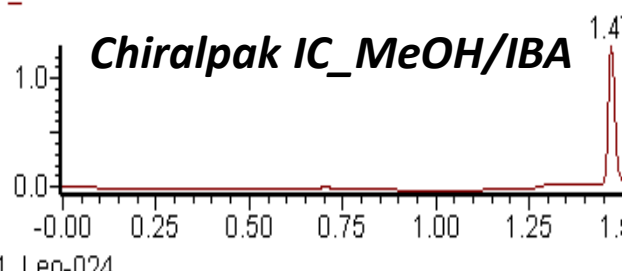

2014-02-11_Leo-024

\section{0: Chiralpak IB_MeOH/IBA}

$0.0 \frac{1}{2}$

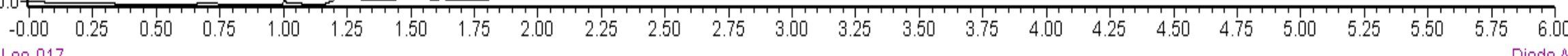
2014-02-11_Leo-017

\section{0 Chiralpak IA_MeOH/IBA}

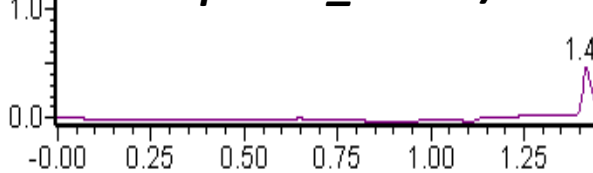

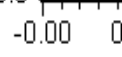
2014-02-11_Leo-010

\section{0: Chiralpak AS MeOH/IBA}

0.0 年

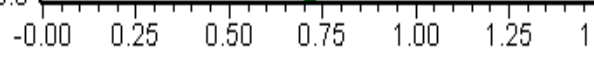
2014-02-11_Leo-003

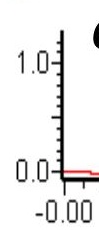

Chiralpak AD_MeOH/IBA

\section{$42^{1.51}$}

Range: 4.981e-1

Range: 1.062

Range: 1.335 
SFC Screening: 1-40\% (0-5 min), 40\% (5-6 min), no post. $3 \mathrm{~mL} / \mathrm{min}, 200 \mathrm{bar}, 40^{\circ} \mathrm{C}$

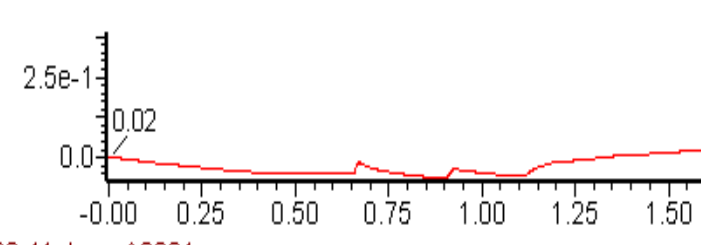

\section{Chiralpak IE_MeOH/IBA}

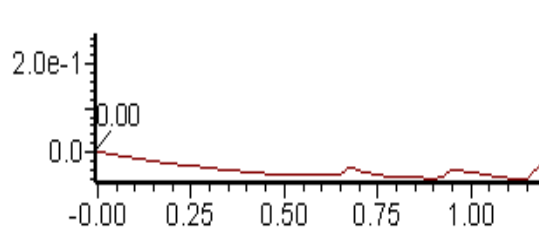

1.93 2.74

Range: $4.496 \mathrm{e}-1$ $\overrightarrow{4}$
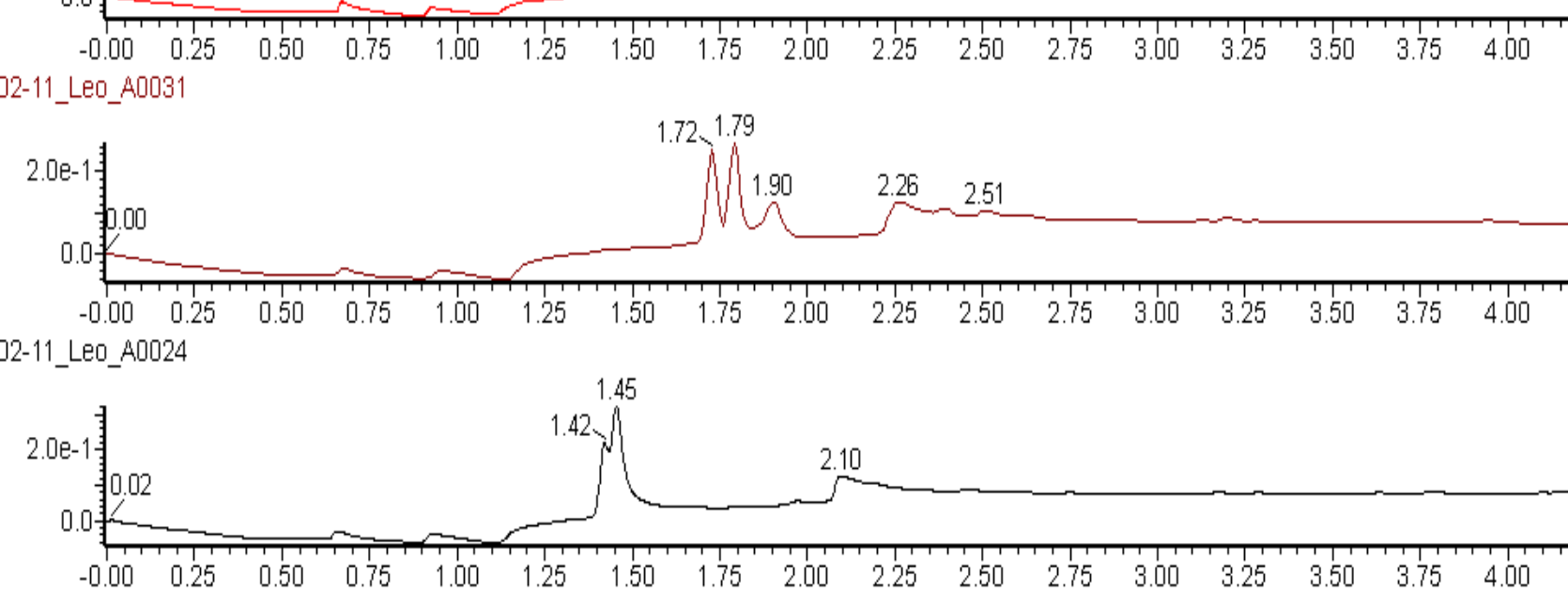

$\begin{array}{llllllll}.25 & 4.50 & 4.75 & 5.00 & 5.25 & 5.50 & 5.75 & 6.00 \\ & & & & & & & \\ & & & \end{array}$

Chiralpak IF_MeOH/IBA Range: $3.295 \mathrm{e}-1$ 014-02-11_Leo_A0017 子 20 2.0e-1 0.02
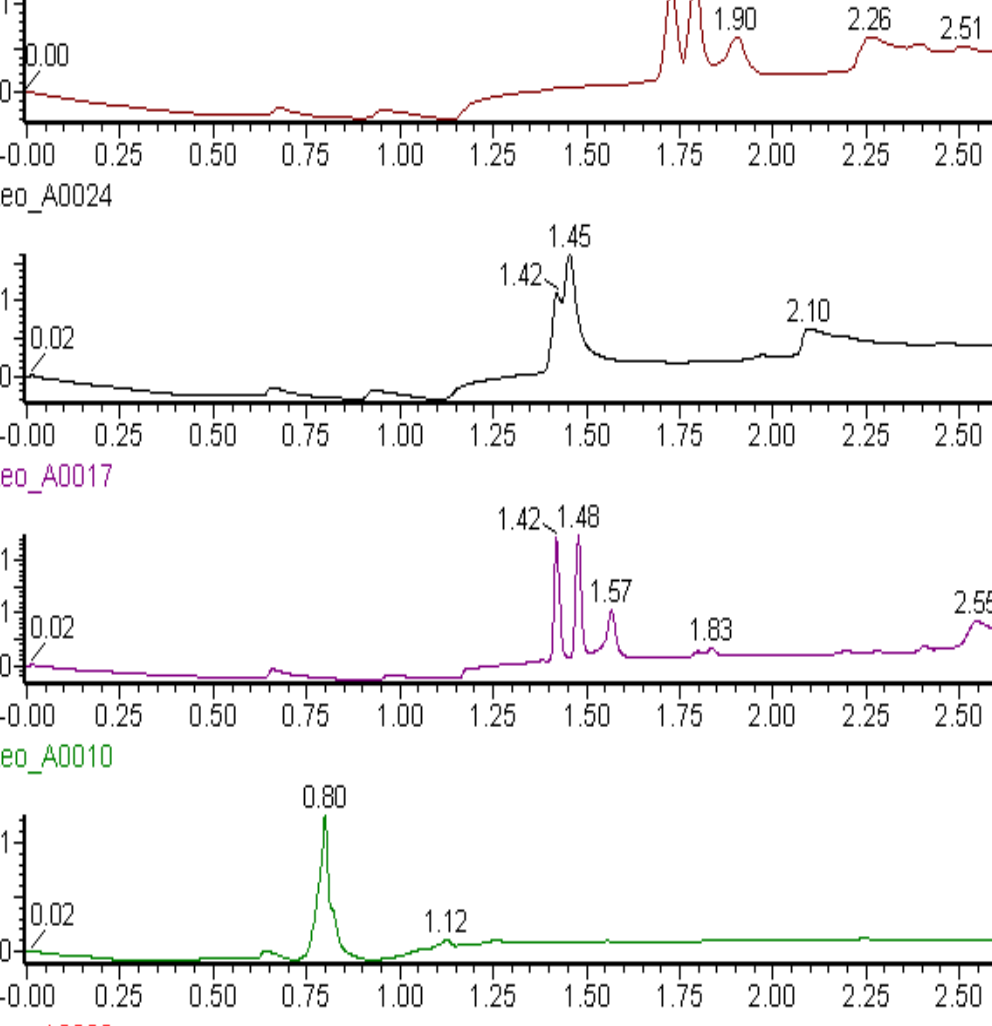

\subsection{0}
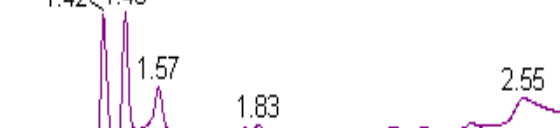

Lux Amylose 2_MeOH/IBA Range: $3.843 \mathrm{e}-1$

Chiralcel OZ_MeOH/IBA Range: $5.486 \mathrm{e}-1$ 2014-02-11_Le0_A0003
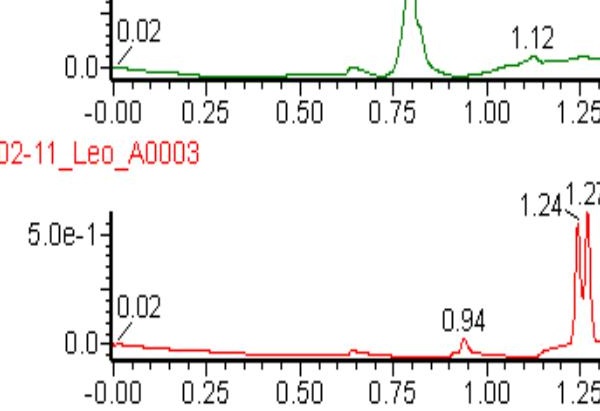

Chiralcel OJ_MeOH/IBA

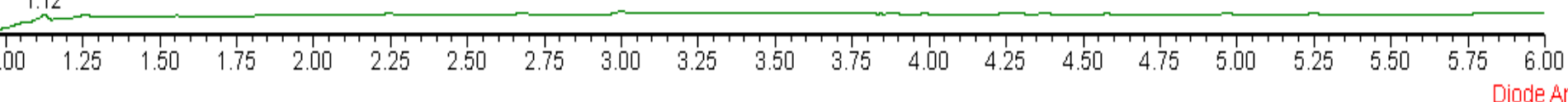

Chiralcel OD_MeOH/IBA Range: 6.564e-1 
SFC Screening: $1-40 \%(0-5 \mathrm{~min}), 40 \%(5-6 \mathrm{~min})$, no post. $3 \mathrm{~mL} / \mathrm{min}, 200 \mathrm{bar}, 40^{\circ} \mathrm{C}$

$$
\text { 5.0e-1 } 1 \text { 龺 }
$$

\section{Lux Cellulose 4_IPA/IBA} 0.00

2014-02-12 Le0-03-

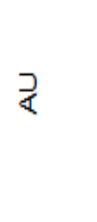

\section{Chiralpak IC_IPA/IBA} 0.01
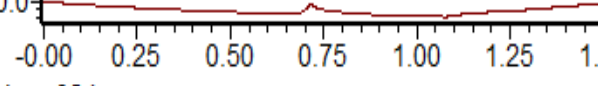

\subsection{1 .711 .80}

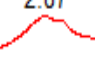

2014-02-12_Le0-024

₹ 5

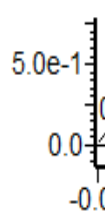

\section{Chiralpak IB_IPA/IBA} 0.01

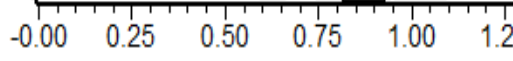

15ा11

2014-02-12_Le0-017

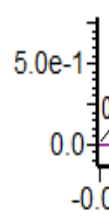

\section{Chiralpak IA IPA/IBA} 0.01

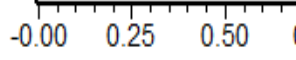

4-02-12_Leo-010
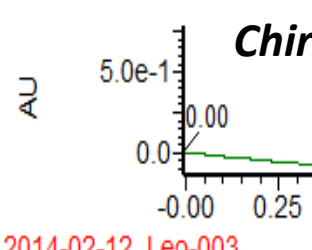

\section{Chiralpak AS_IPA/IBA}
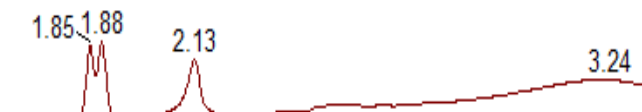

Range: $6.05 \mathrm{e}-1$ 


\section{SFC Screening: 1-40\% (0-5 $\mathrm{min}), 40 \%(5-6 \mathrm{~min})$, no post. $3 \mathrm{~mL} / \mathrm{min}, 200 \mathrm{bar}, 40^{\circ} \mathrm{C}$}

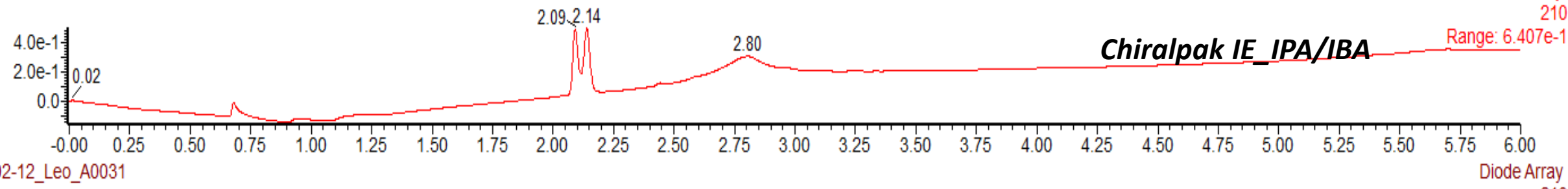

014-02-12_Leo_A0031
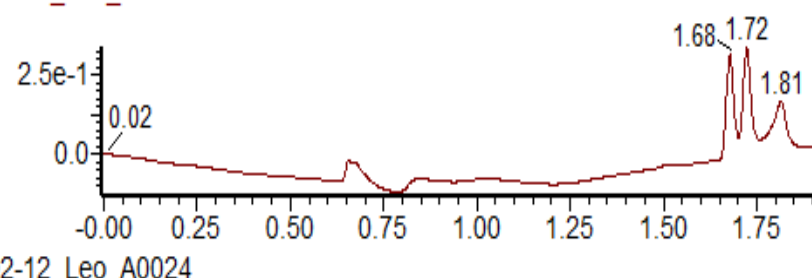

Chiralpak IF_IPA/IBA Range: $4.595 \mathrm{e}-1$ 2014-02-12_Leo_A0024
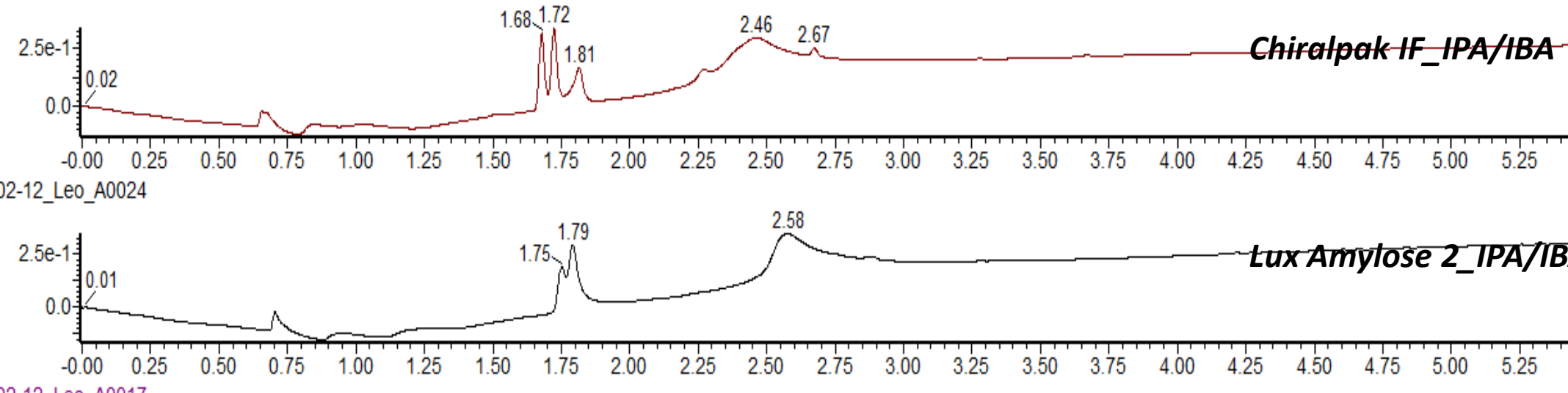
2014-02-12_Leo_A0017
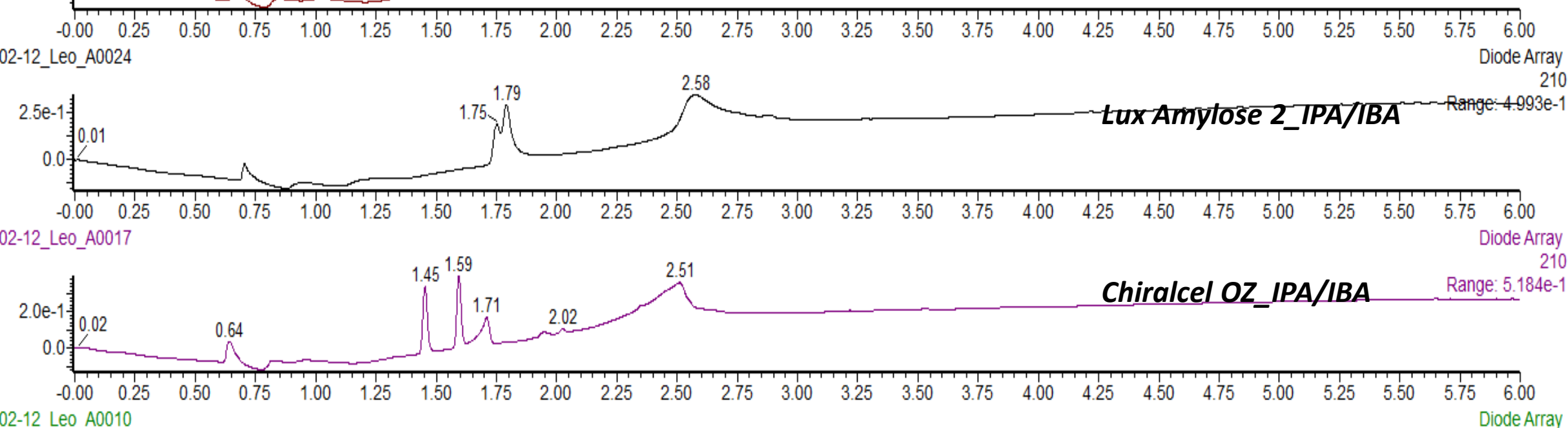
2014-02-12_Leo_A0010
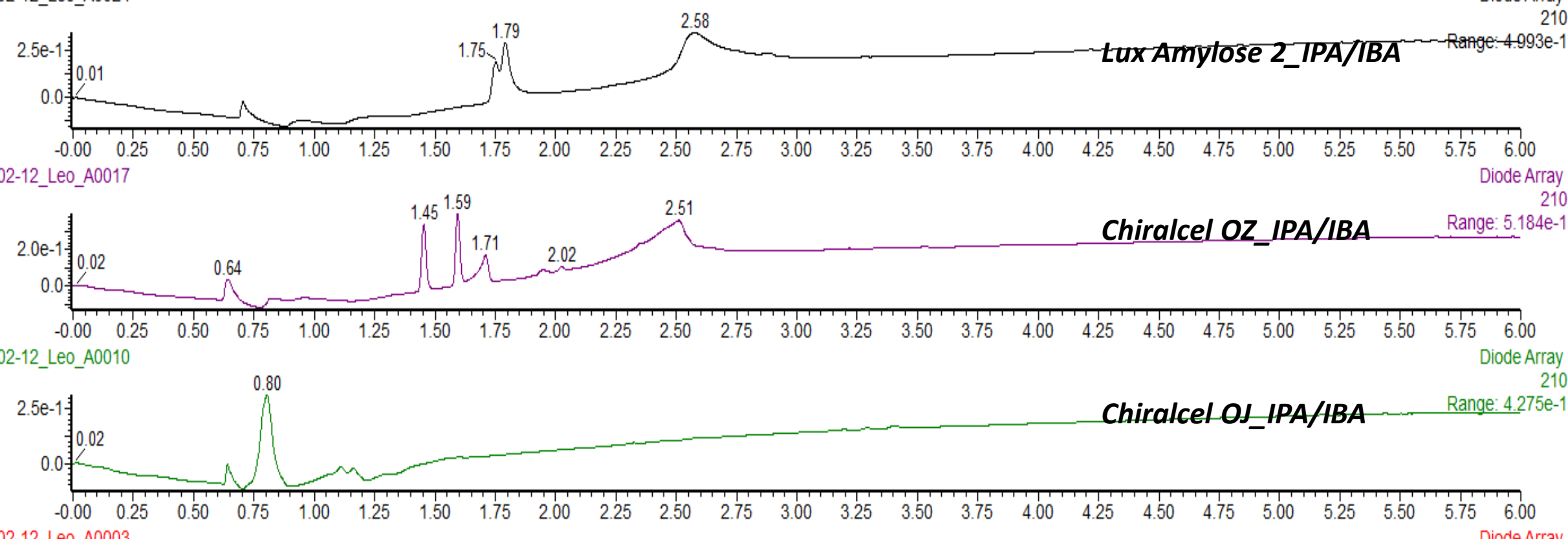
2014-02-12_Leo_A0003 
SFC Screening: $1-40 \%(0-5 \mathrm{~min}), 40 \%(5-6 \mathrm{~min})$, no post. $3 \mathrm{~mL} / \mathrm{min}, 200 \mathrm{bar}, 40^{\circ} \mathrm{C}$

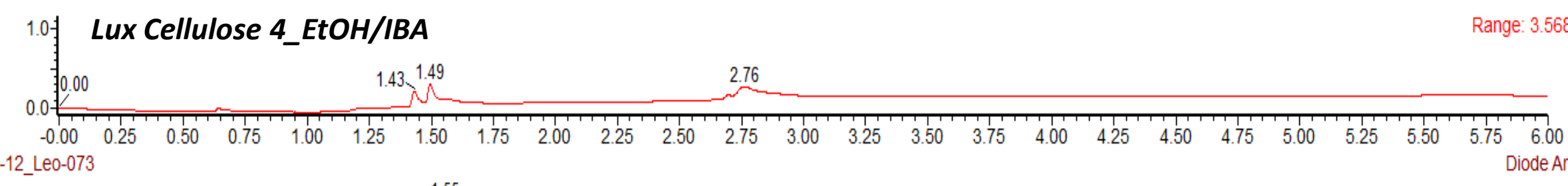

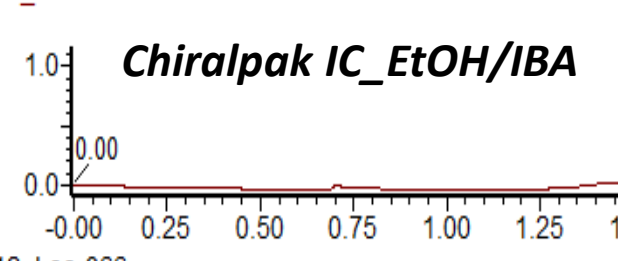

-0.00
Leo- 066

\section{0f Chiralpak IB_EtOHy/ABA}

0.0

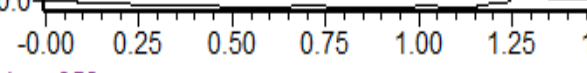

2014-02-12_Leo-059
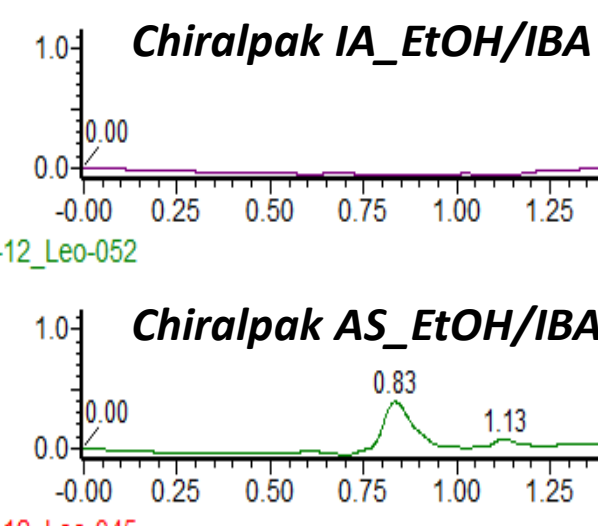

201 $-0.00 \quad 0.25$

$$
\stackrel{1.09}{0.0 \frac{7}{0.01}}
$$

Chiralpak AD_EtOH/IBA $A^{47} 1.55$

\section{$1.48,1.53$} 2.38 
SFC Screening: 1-40\% (0-5 min), 40\% (5-6 min), no post. $3 \mathrm{~mL} / \mathrm{min}, 200 \mathrm{bar}, 40^{\circ} \mathrm{C}$

S50

AMH Imine
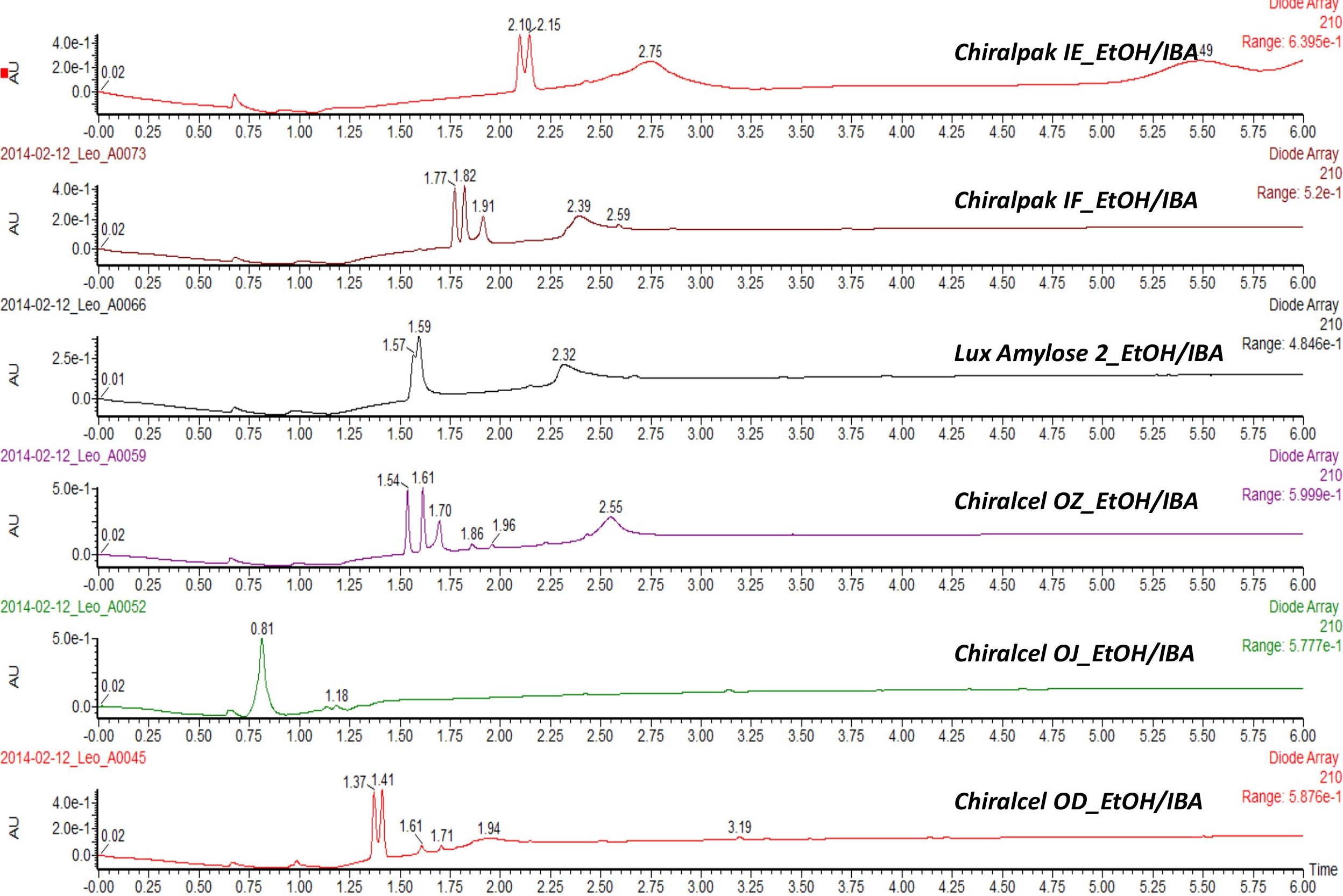


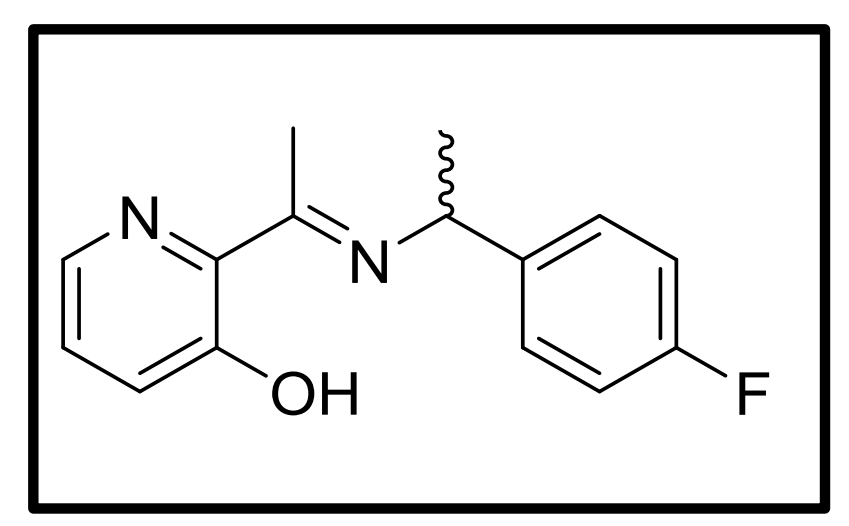

Primary Chiral SFC Screen

12 columns, 3 solvents

(with and without $25 \mathrm{mM}$ IBA) 
SFC Screening: $1-40 \%(0-5 \mathrm{~min}), 40 \%(5-6 \mathrm{~min})$, no post. $3 \mathrm{~mL} / \mathrm{min}, 200 \mathrm{bar}, 40^{\circ} \mathrm{C}$

S52

1.0 : Lux Cellulose 4_MeOH/IBA

Diode Array

Range: $2.28 \mathrm{e}-1$

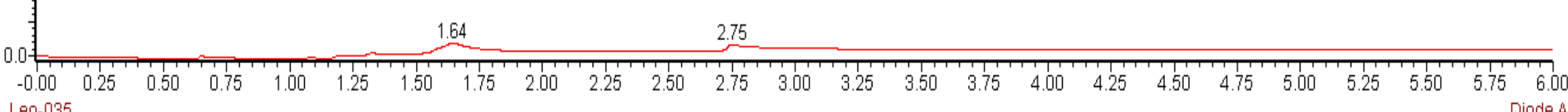

2014-02-11_Leo-035
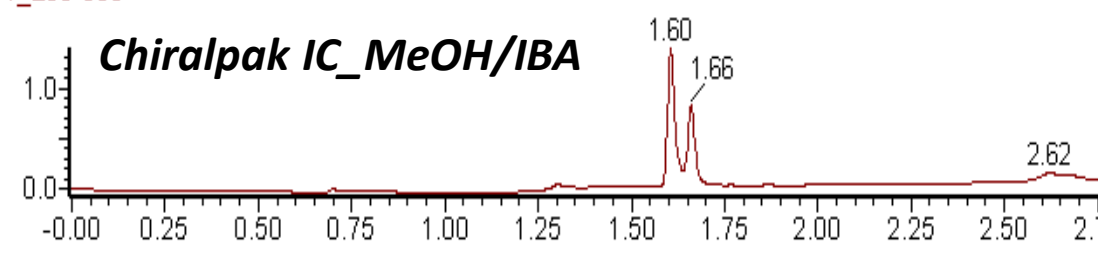
2014-02-11_Le0-028

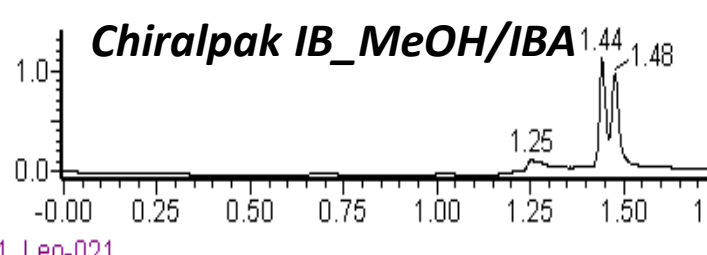

2014-02-11_Leo-021

\section{0: Chiralpak IA_MeOH/IBA}

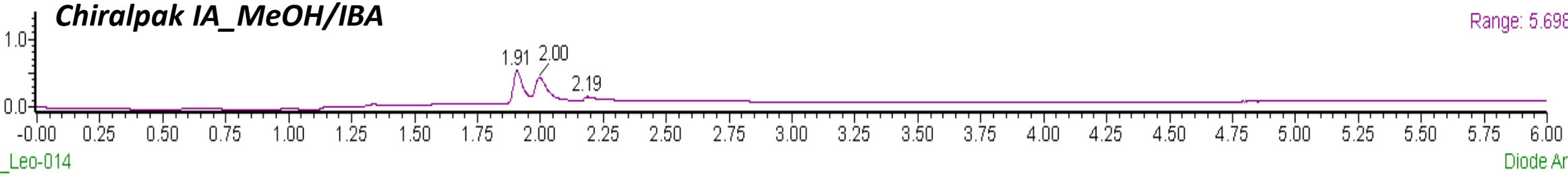
2014-02-11_Leo-014

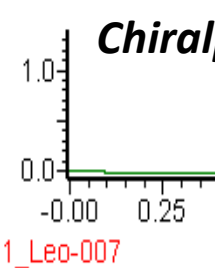

Chiralpak AS_MeOH/IBA

Range: $6.158 \mathrm{e}-1$

Chiralpak AD_MeOH/IBA 
SFC Screening: 1-40\% (0-5 min), 40\% (5-6 min), no post. $3 \mathrm{~mL} / \mathrm{min}, 200 \mathrm{bar}, 40^{\circ} \mathrm{C}$
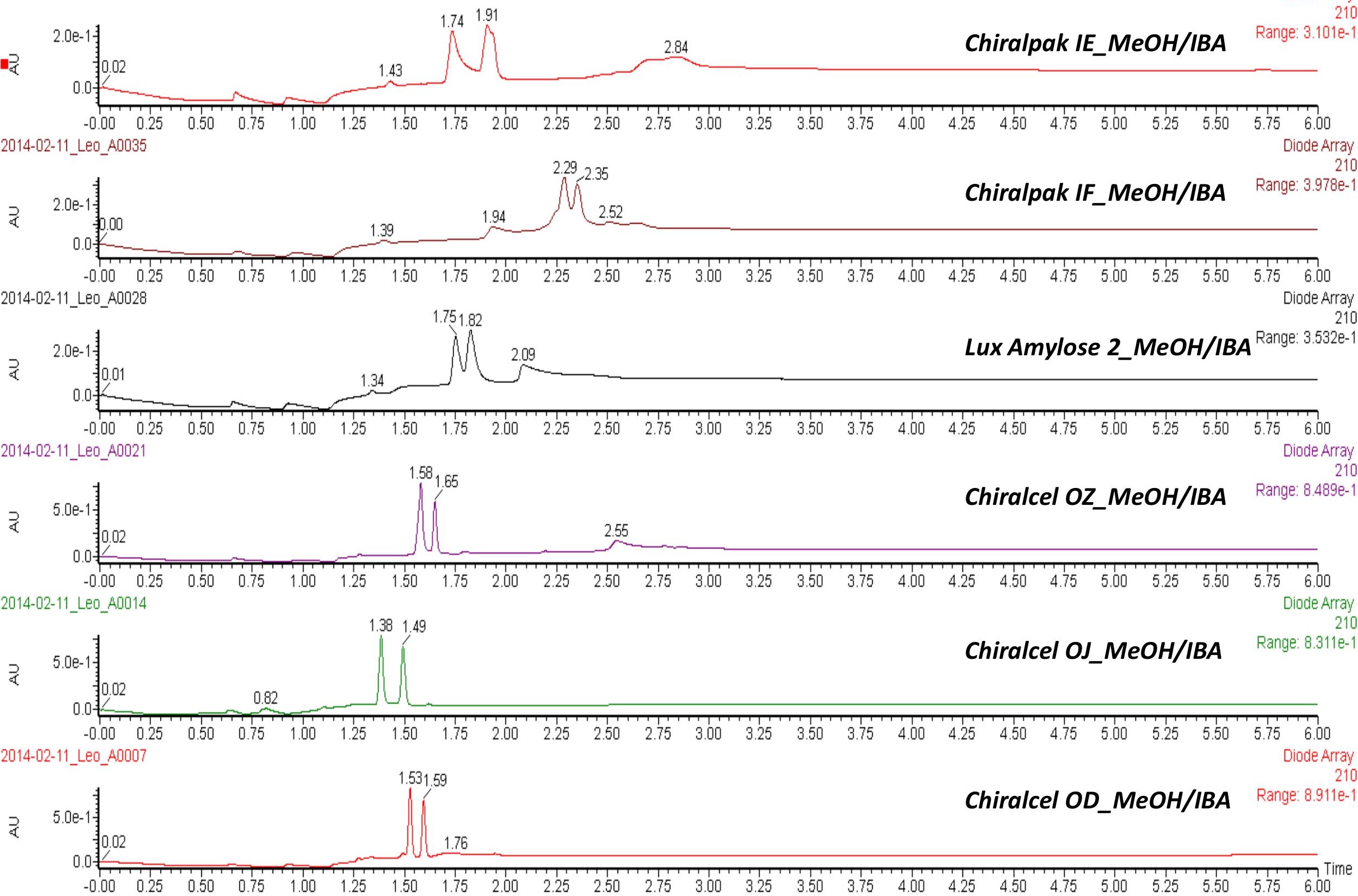
SFC Screening: $1-40 \%(0-5 \mathrm{~min}), 40 \%(5-6 \mathrm{~min})$, no post. $3 \mathrm{~mL} / \mathrm{min}, 200 \mathrm{bar}, 40^{\circ} \mathrm{C}$

S54

\section{0견 Lux Cellulose 4_IPA/IBA}

Diode Array

Range: $5.244 \mathrm{e}-1$

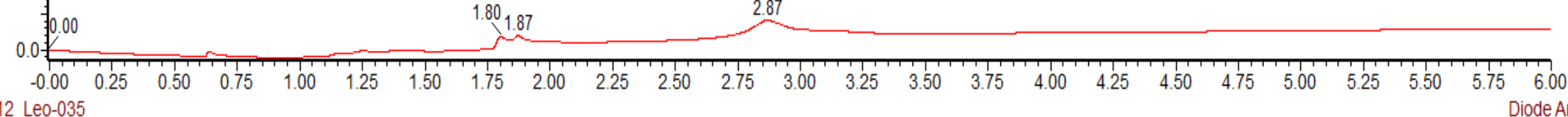

2014-02-12_Leo-035
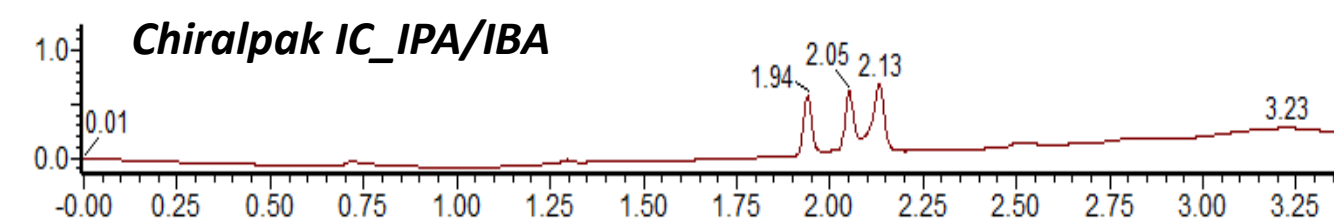
2014-02-12_Le0-028

\subsection{Chiralpak IB_IPA/IBA}
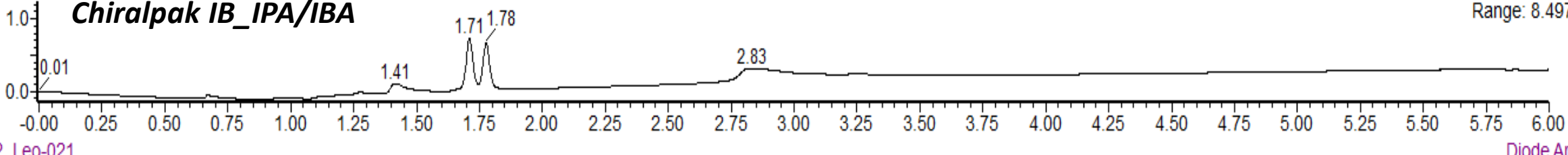

2014-02-12_Leo-021

\& $\quad 1.0$ 年
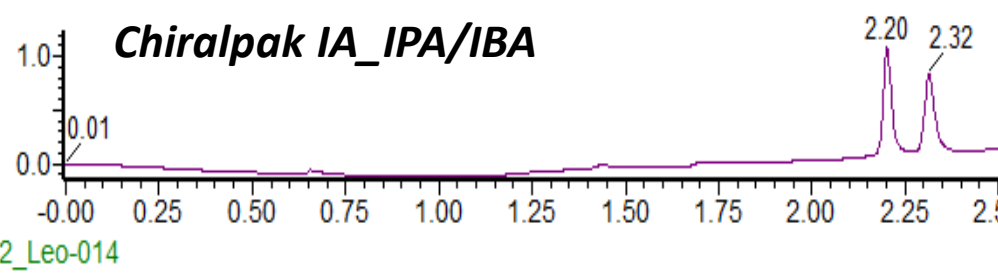

Diode Array

2014-02-12_Le0-014

\subsection{Chiralpak AS_IPA/IBA}

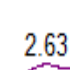

Range: 1.199
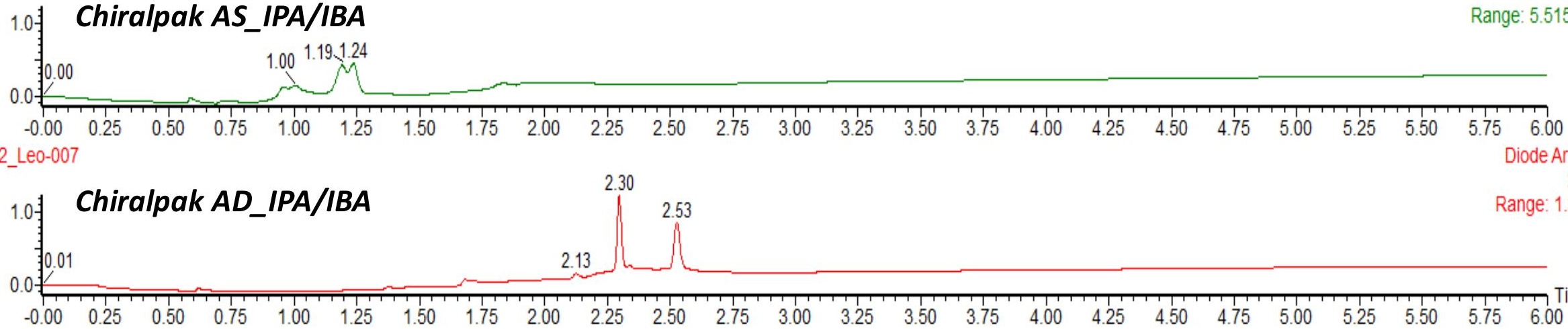

\section{Chiralpak AD_IPA/IBA}

Range: 1.333

$\begin{array}{ccccccccccccccccccccccccc}-0.00 & 0.25 & 0.50 & 0.75 & 1.00 & 1.25 & 1.50 & 1.75 & 2.00 & 2.25 & 2.50 & 2.75 & 3.00 & 3.25 & 3.50 & 3.75 & 4.00 & 4.25 & 4.50 & 4.75 & 5.00 & 5.25 & 5.50 & 5.75 & 6.00\end{array}$




\section{SFC Screening: 1-40\% (0-5 $\mathrm{min}), 40 \%(5-6 \mathrm{~min})$, no post. $3 \mathrm{~mL} / \mathrm{min}, 200 \mathrm{bar}, 40^{\circ} \mathrm{C}$}

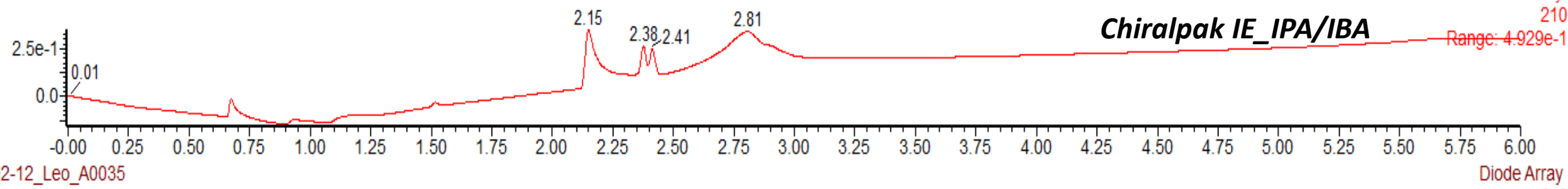
2014-02-12_Leo_A0035
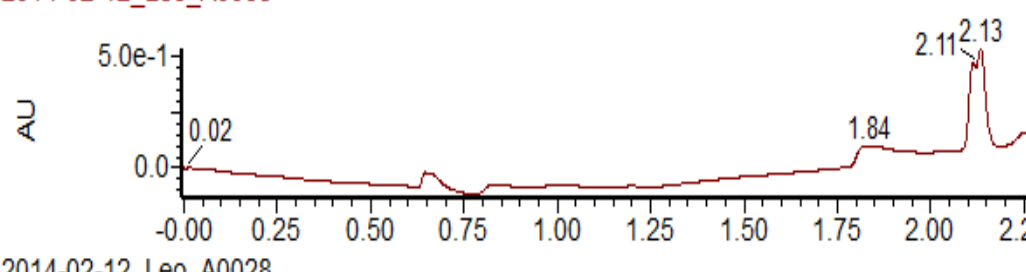

Chiralpak IF_IPA/IBA

Range: $6.525 \mathrm{e}-1$ 2014-02-12_Leo_A0028 $\begin{array}{ll}0.00 & 0.25 \\ 0.0028 & 1\end{array}$ ২ 2

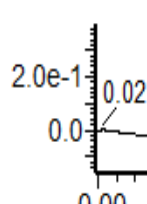
1.84

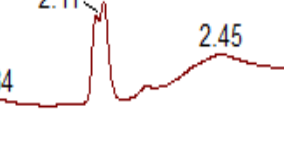
2014-02-12_Leo_A0021
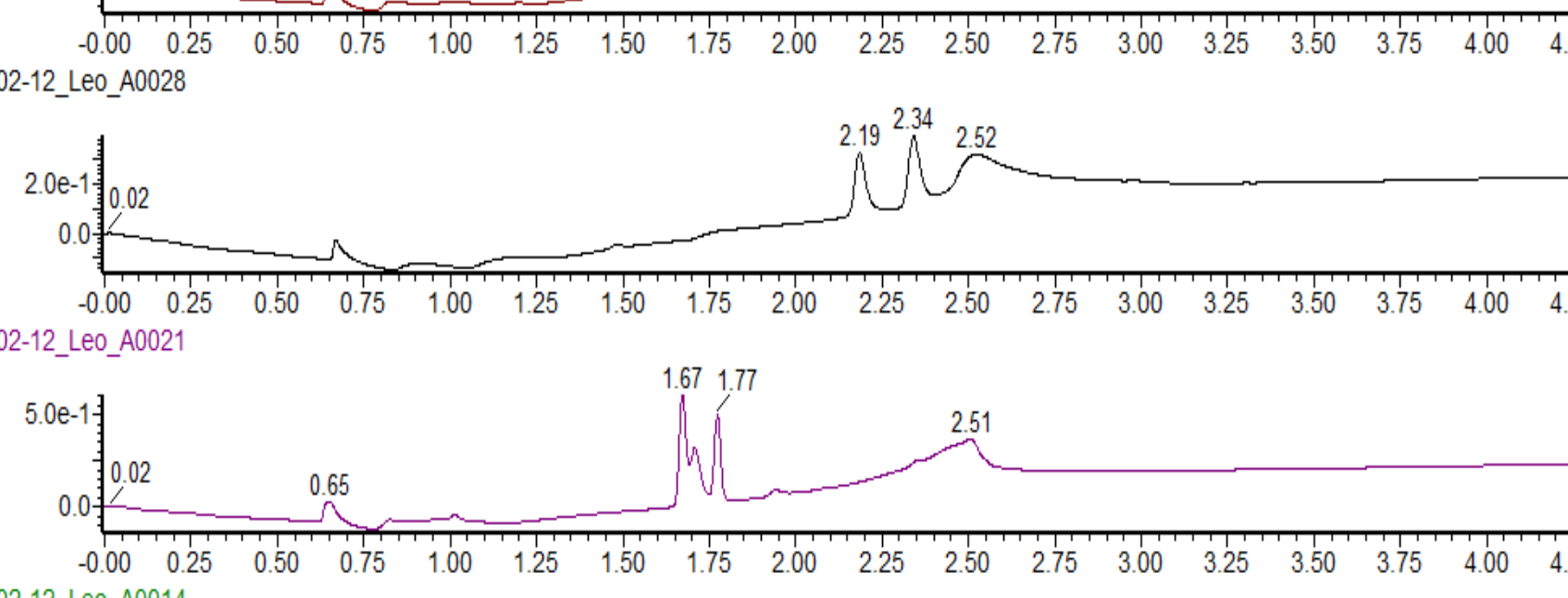

$\begin{array}{rrrrrrrr}4.25 & 4.50 & 4.75 & 5.00 & 5.25 & 5.50 & 5.75 & 6.00 \\ & & & & & & & \text { Diode A }\end{array}$ Leo_A0014

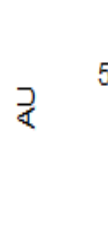
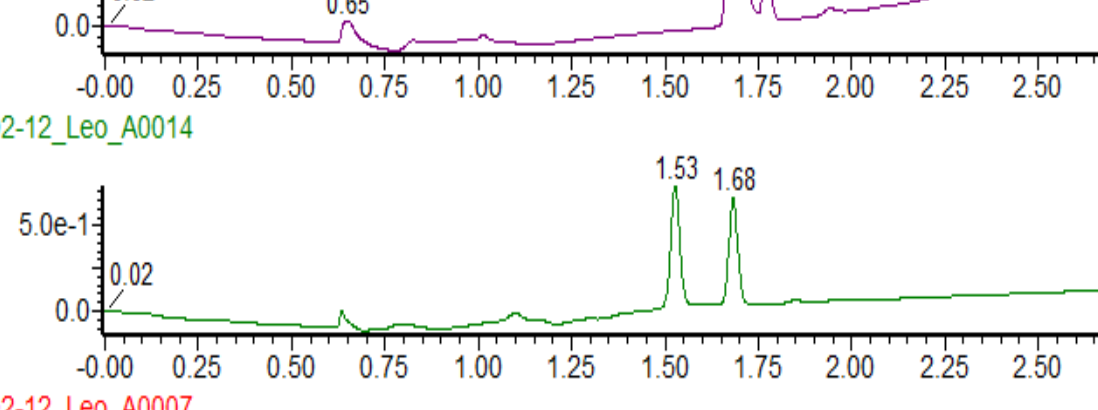

\section{Lux Amylose 2_IPA/IBA}

Range: $5.462 \mathrm{e}-1$

\section{4-02-12_Leo_A0007}

\& $5.0 \mathrm{e}-1$

\section{Chiralcel OJ_IPA/IBA}

Chiralcel OZ_IPA/IBA

Range: $7.268 \mathrm{e}-1$

56.00

Diode Array

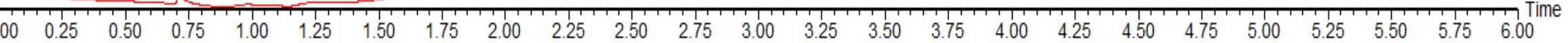


SFC Screening: $1-40 \%(0-5 \mathrm{~min}), 40 \%(5-6 \mathrm{~min})$, no post. $3 \mathrm{~mL} / \mathrm{min}, 200 \mathrm{bar}, 40^{\circ} \mathrm{C}$

S56

\section{0}

Diode Array

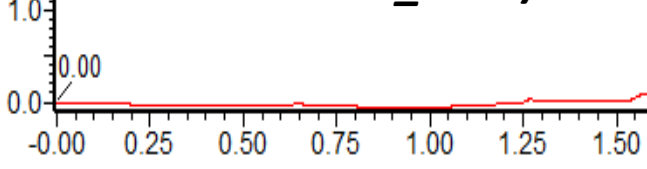

1.66 2014-02-12_Leo-077

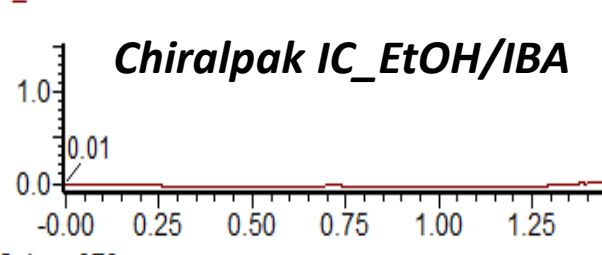

1.75

(2.76

2014-02-12_Leo-070
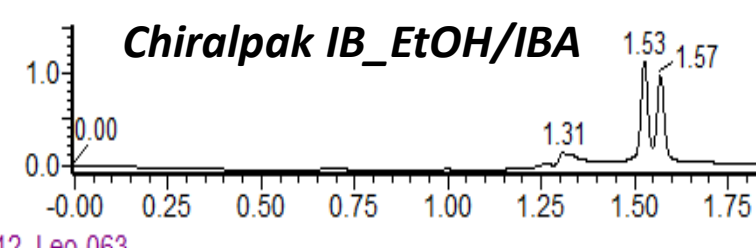

$\begin{array}{lllllllllll}2.00 & 2.25 & 2.50 & 2.75 & 3.00 & 3.25 & 3.50 & 3.75 & 4.00 & 4.25 & 4.50\end{array}$

$\begin{array}{lllllll}.50 & 4.75 & 5.00 & 5.25 & 5.50 & 5.75 & 6.00\end{array}$

_Leo-063
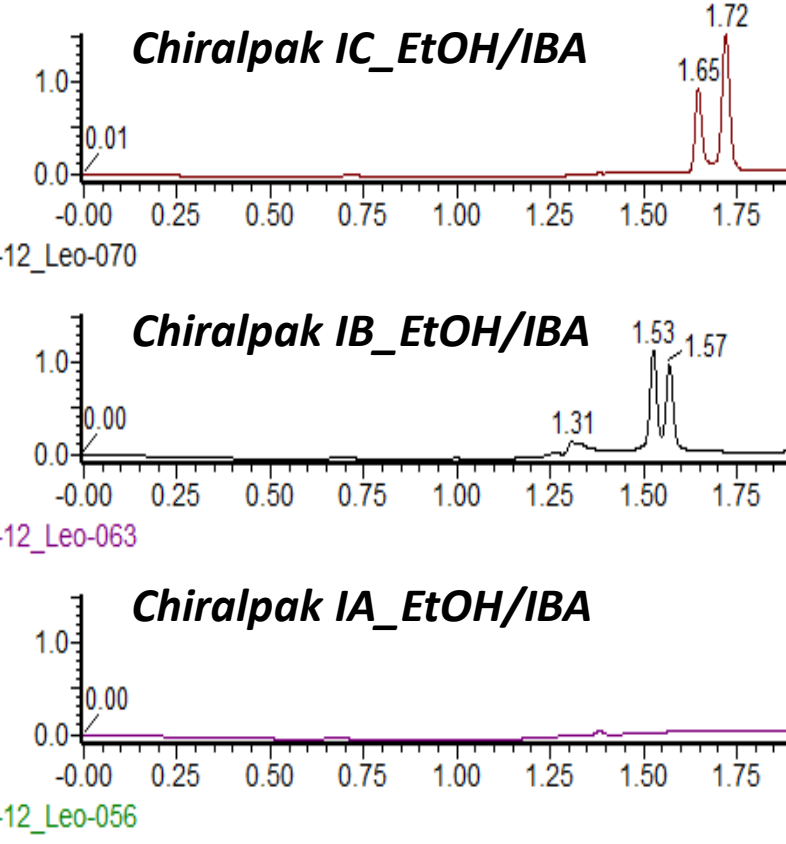

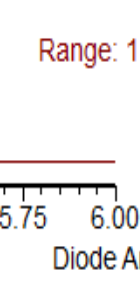

Range: $3.324 \mathrm{e}-1$

2014-02-12_Le0-05

\subsection{Chiralpak AS_EtOH/IBA}

2.84
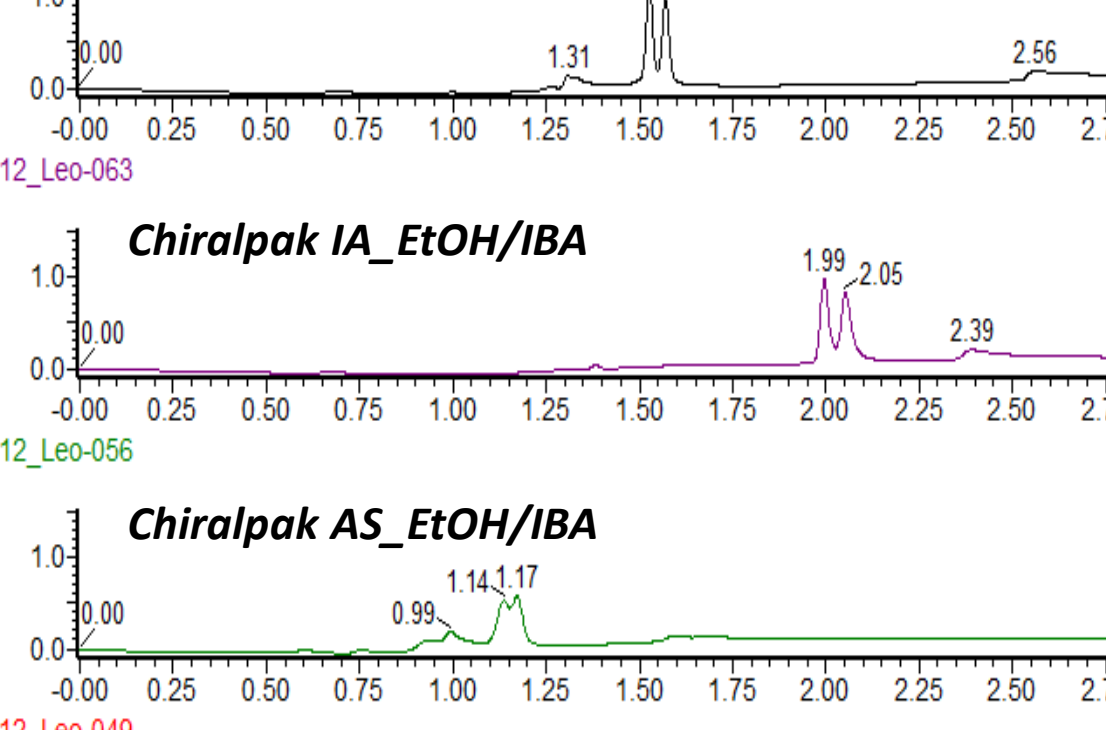

Range: 1.185 2014-02-12_Leo-049

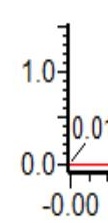

\section{Chiralpak AD_EtOH/IBA}


SFC Screening: 1-40\% (0-5 $\mathrm{min}), 40 \%(5-6 \mathrm{~min})$, no post. $3 \mathrm{~mL} / \mathrm{min}, 200 \mathrm{bar}, 40^{\circ} \mathrm{C}$

S57

4-F MBA Imine

Diode Array

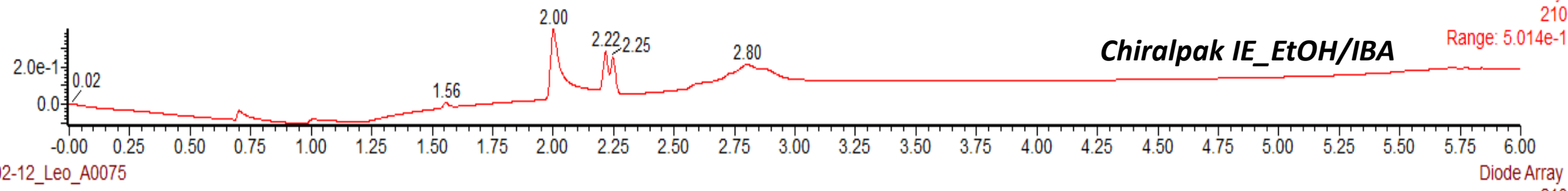
2014-02-12_Leo_A0075

२

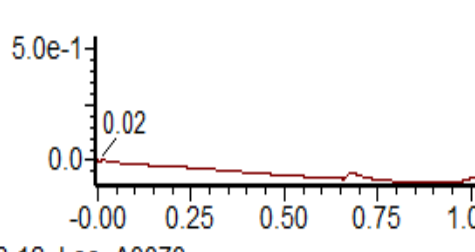

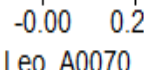

2014-02-12_LE_A0070_.94 2.06

$\frac{2}{2}$

2.5e-1

-0.00

1.0
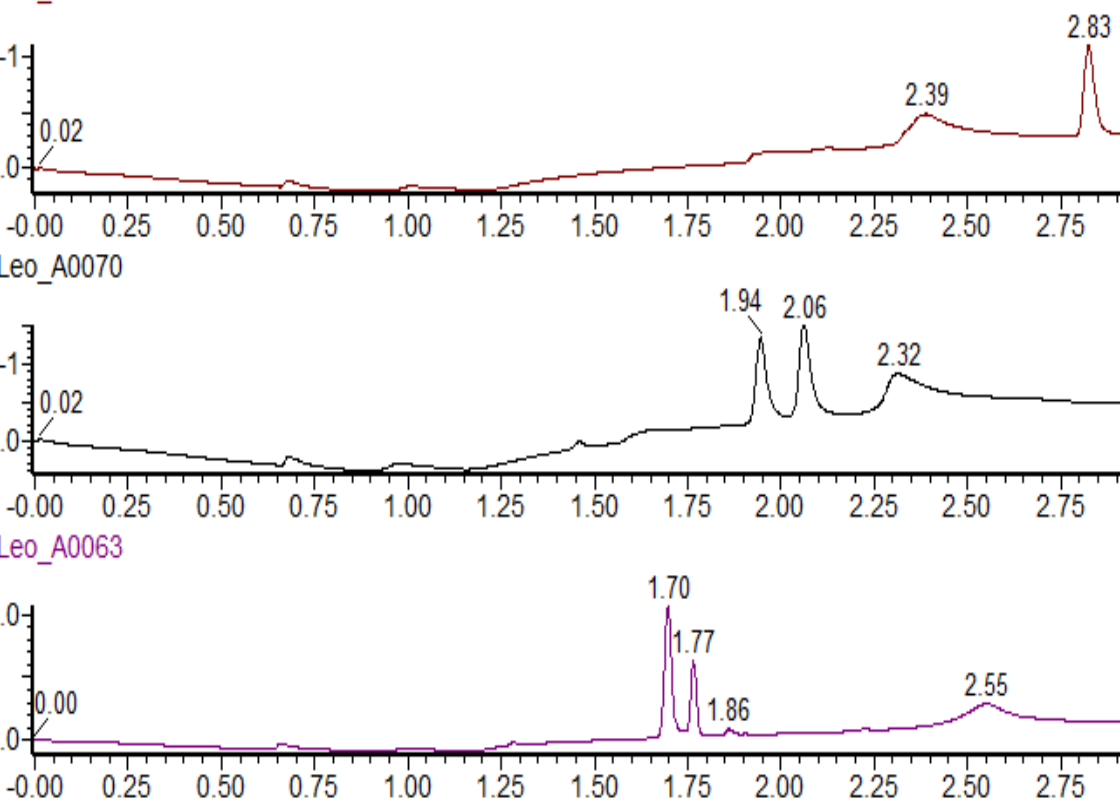

Chiralpak IF_EtOH/IBA

Range: $6.416 \mathrm{e}-1$ 2014-02-12_Leo_A0056

子

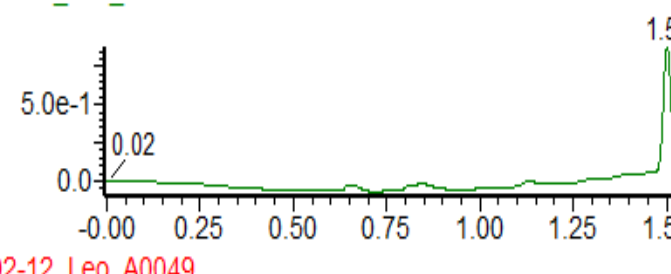

\section{.501 .63}

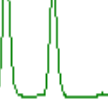

Chiralcel OJ_EtOH/IBA

Lux Amylose 2_EtOH/IBA

$\begin{array}{ll}75 & 6.00\end{array}$

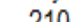

2014-02-12_Leo_A0049

己 $5.0 \mathrm{e}-1$ 蜶

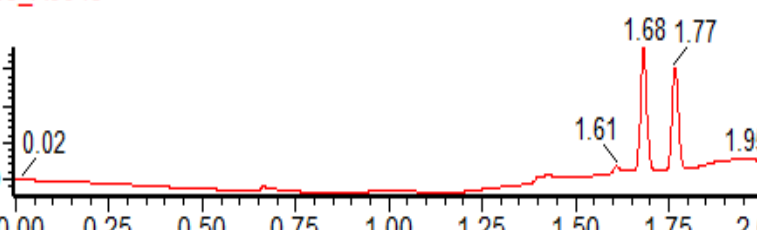

Chiralcel OZ_EtOH/IBA

6.00
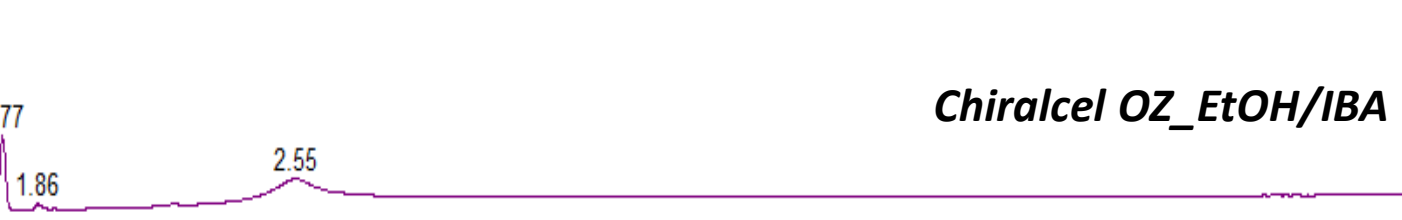


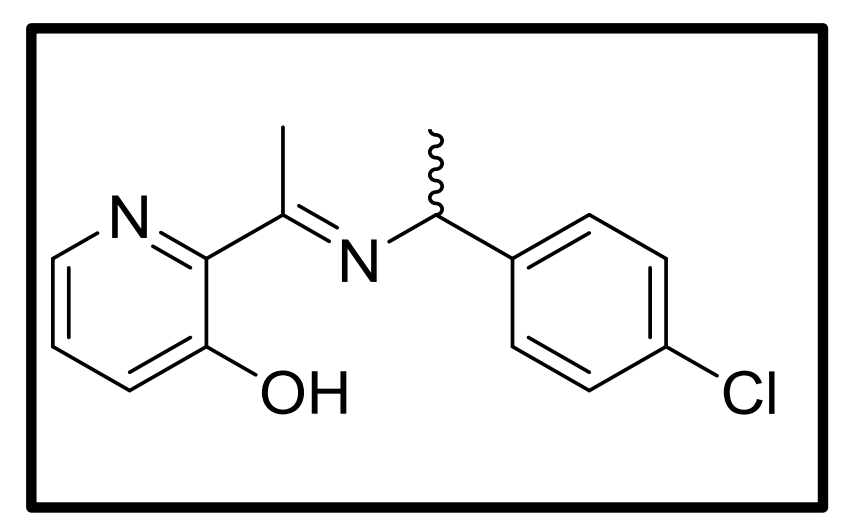

Primary Chiral SFC Screen

12 columns, 3 solvents

(with and without $25 \mathrm{mM}$ IBA) 
SFC Screening: 1-40\% (0-5 min), 40\% (5-6 min), no post. $3 \mathrm{~mL} / \mathrm{min}, 200 \mathrm{bar}, 40^{\circ} \mathrm{C}$

S59

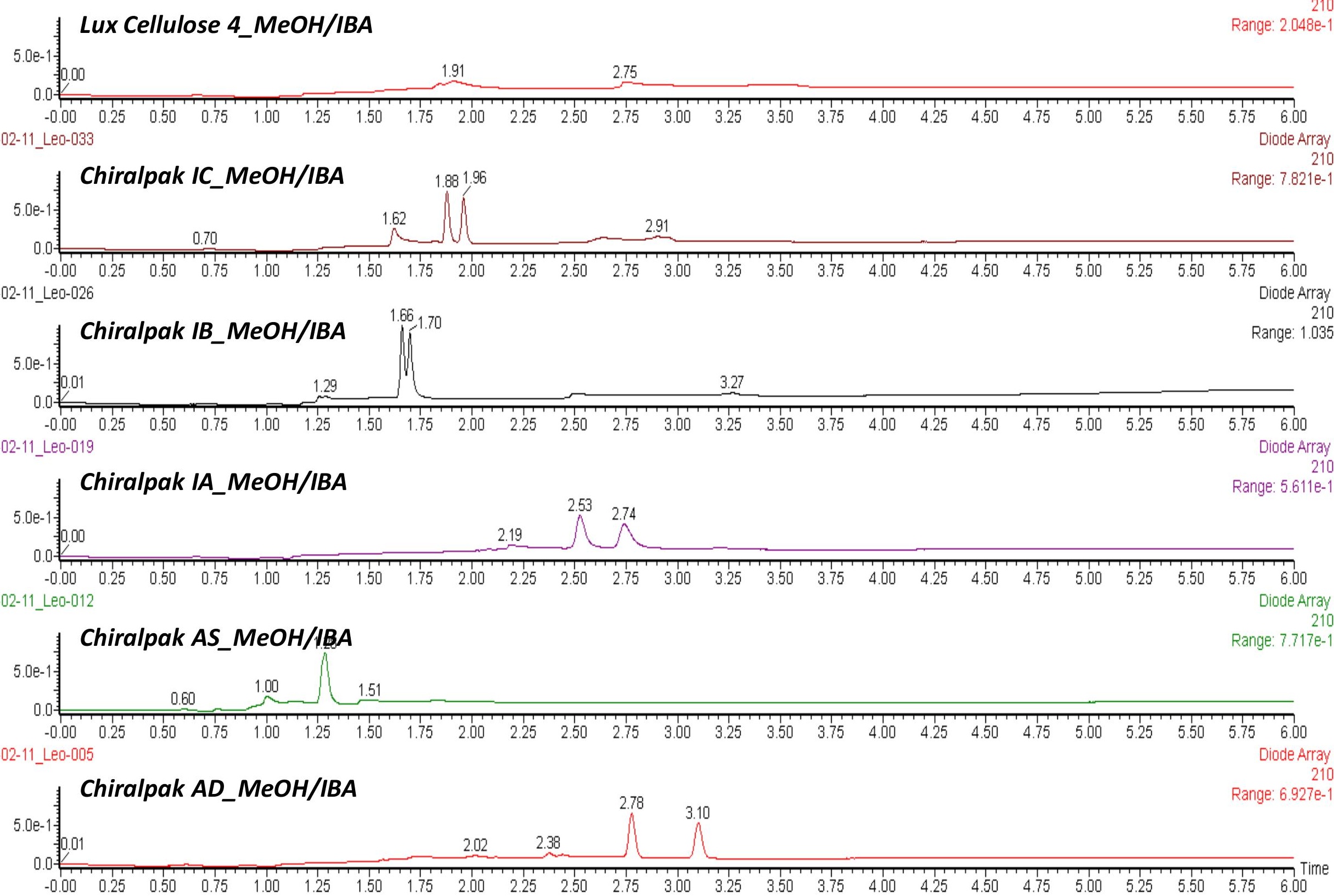


SFC Screening: $1-40 \%(0-5 \mathrm{~min}), 40 \%(5-6 \mathrm{~min})$, no post. $3 \mathrm{~mL} / \mathrm{min}, 200 \mathrm{bar}, 40^{\circ} \mathrm{C}$

S60

4-Cl MBA Imine

Diode Array
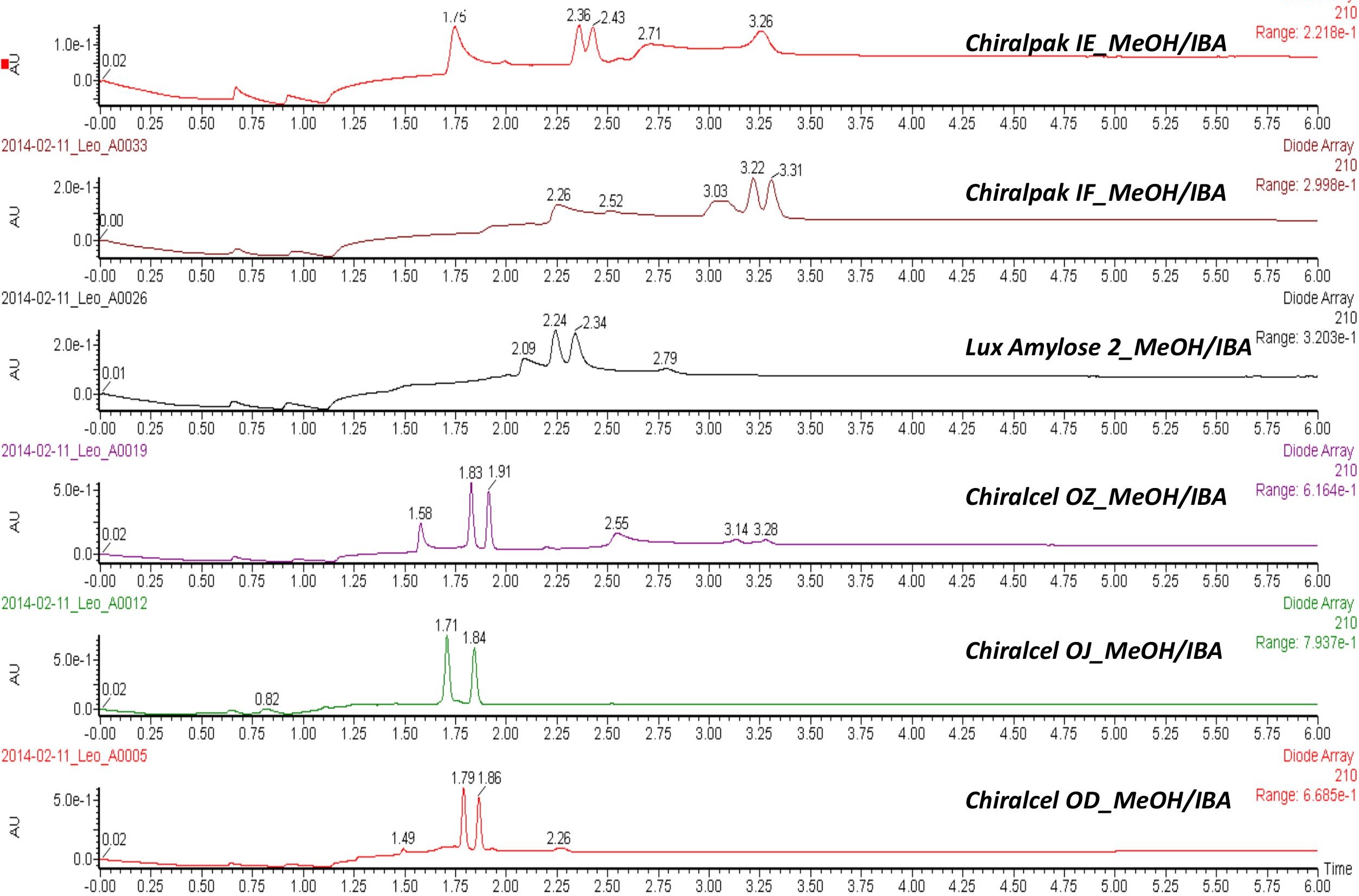
SFC Screening: $1-40 \%(0-5 \mathrm{~min}), 40 \%(5-6 \mathrm{~min})$, no post. $3 \mathrm{~mL} / \mathrm{min}, 200 \mathrm{bar}, 40^{\circ} \mathrm{C}$

S61
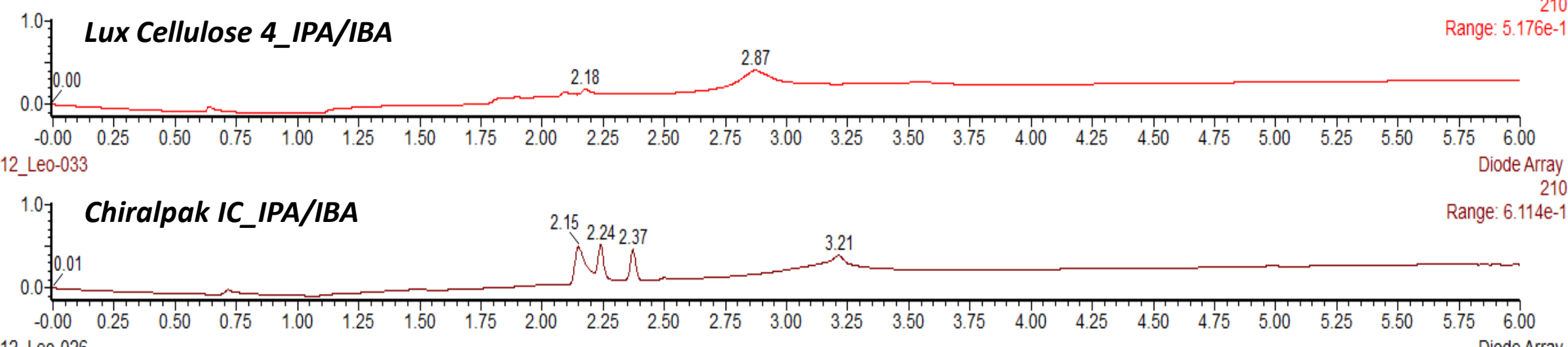
2014-02-12_Le0-026
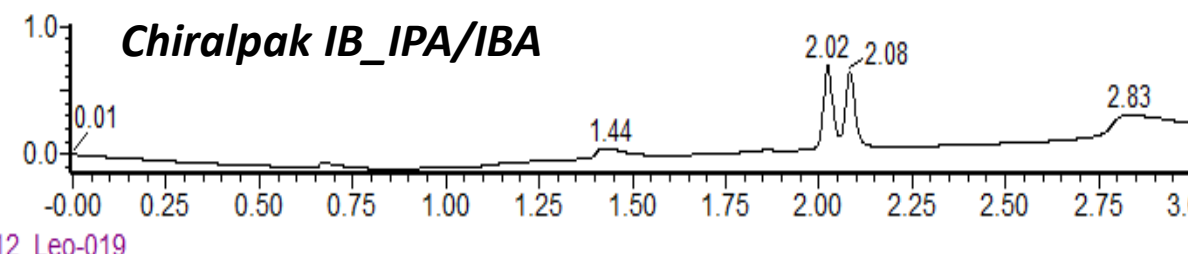

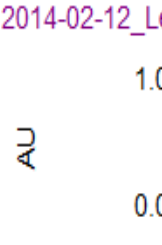
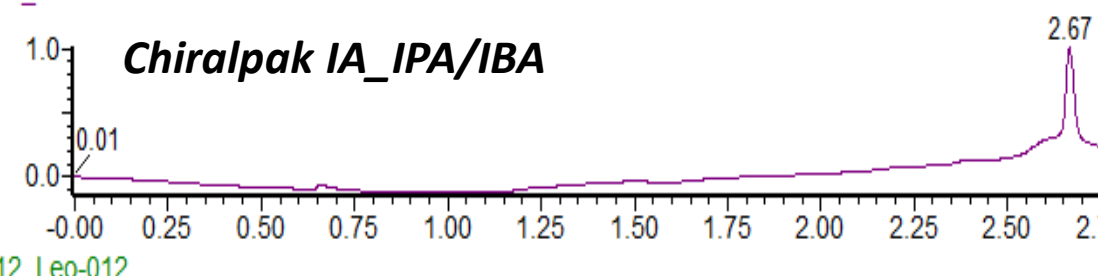

2014-02-12_Leo-012

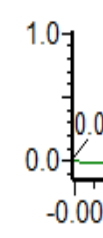

0.0

\section{Chiralpak AS_IPA/IBA} 2014-02-12_Le0-005

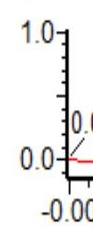

\section{Chiralpak AD_IPA/IBA} 0.01 
SFC Screening: 1-40\% (0-5 $\mathrm{min}), 40 \%(5-6 \mathrm{~min})$, no post. $3 \mathrm{~mL} / \mathrm{min}, 200 \mathrm{bar}, 40^{\circ} \mathrm{C}$

S62

4-Cl MBA Imine
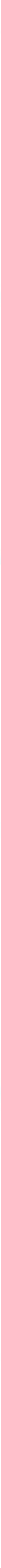
SFC Screening: $1-40 \%(0-5 \mathrm{~min}), 40 \%(5-6 \mathrm{~min})$, no post. $3 \mathrm{~mL} / \mathrm{min}, 200 \mathrm{bar}, 40^{\circ} \mathrm{C}$

S63
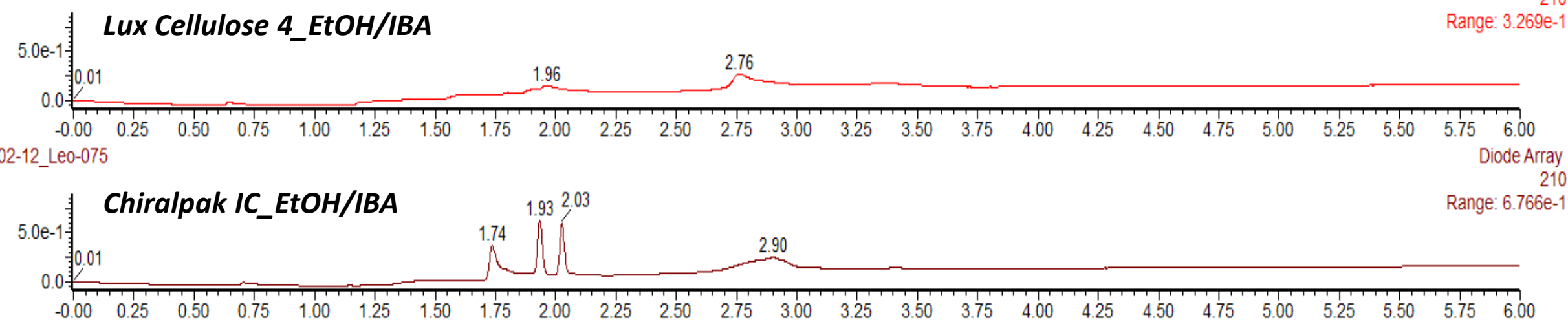

\section{Chiralpak IC_EtOH/IBA}

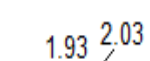

Range: $6.766 \mathrm{e}-1$ 2014-02-12_Le0-068

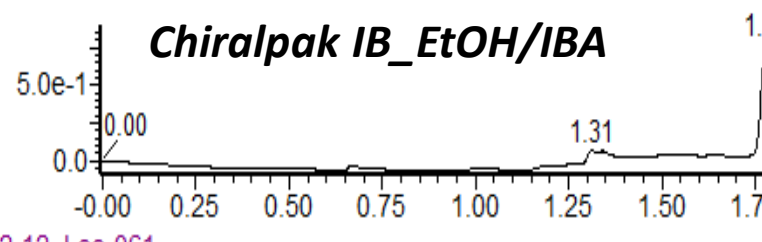
2014-02-12_Le0-061

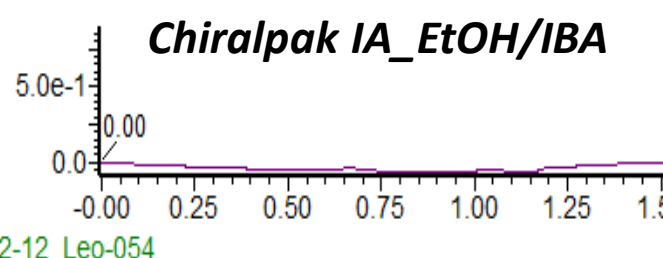
2014-02-12_Le0-054

$$
5.0 \mathrm{e}-1 \text { 章 }
$$

\section{Chiralpak AS_EtOH/JBA7}

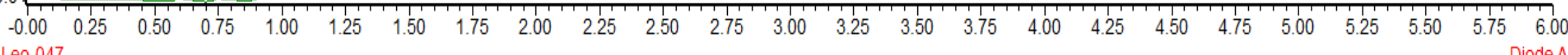
2014-02-12_Le0-047

$$
\text { 5.0e-1 }
$$

\section{Chiralpak AD_EtOH/IBA}


SFC Screening: 1-40\% (0-5 $\mathrm{min}), 40 \%(5-6 \mathrm{~min})$, no post. $3 \mathrm{~mL} / \mathrm{min}, 200 \mathrm{bar}, 40^{\circ} \mathrm{C}$

S64

4-Cl MBA Imine
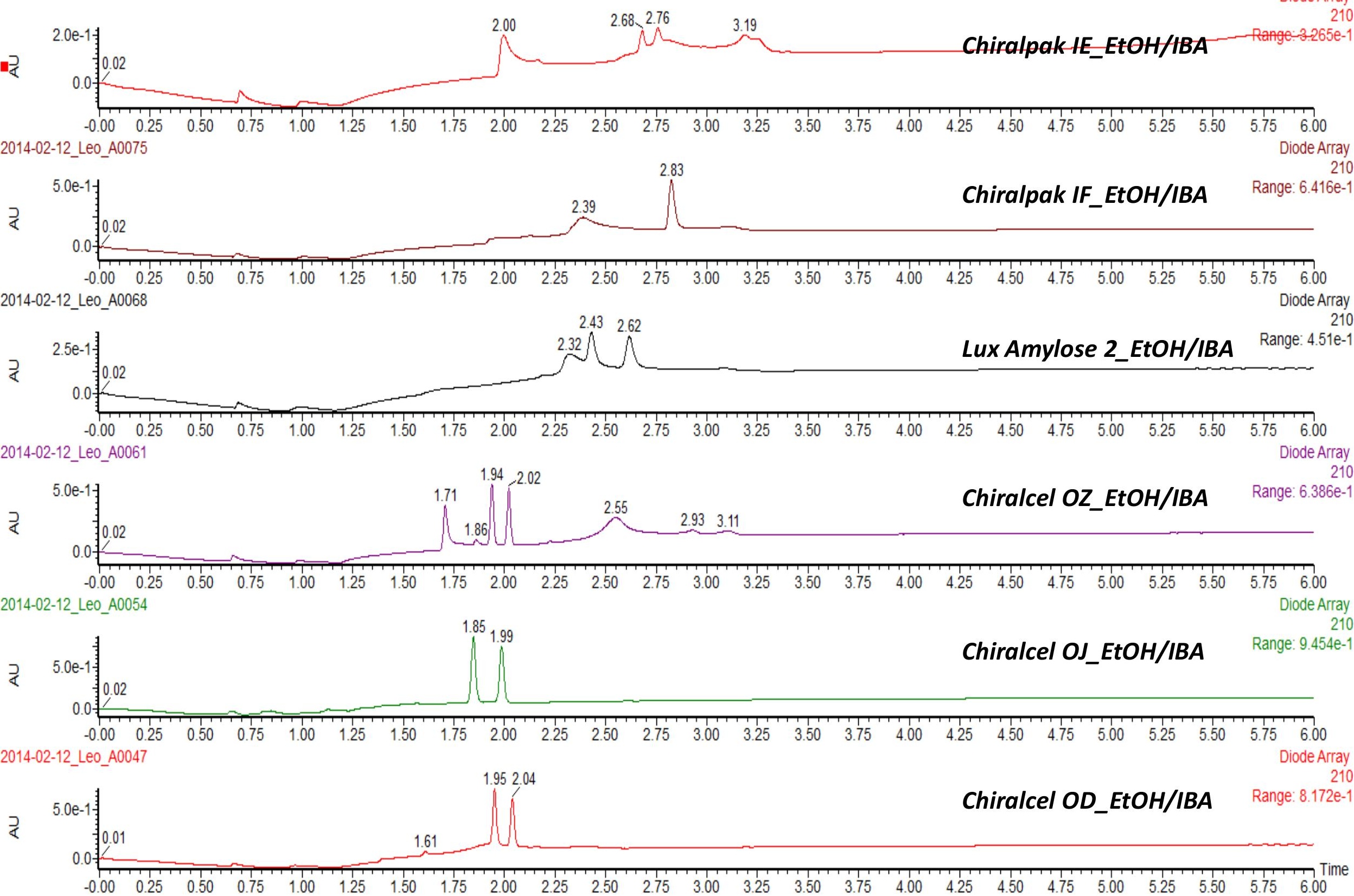


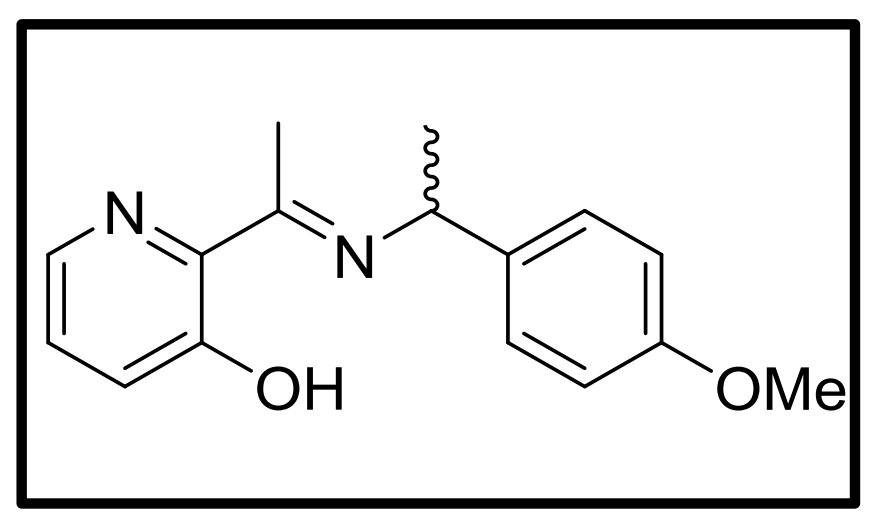

Primary Chiral SFC Screen

12 columns, 3 solvents

(with and without 25 mM IBA) 
SFC Screening: $1-40 \%(0-5 \mathrm{~min}), 40 \%(5-6 \mathrm{~min})$, no post. $3 \mathrm{~mL} / \mathrm{min}, 200 \mathrm{bar}, 40^{\circ} \mathrm{C}$

S66
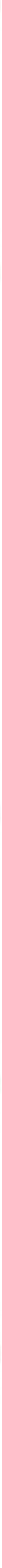
SFC Screening: 1-40\% (0-5 min), 40\% (5-6 min), no post. $3 \mathrm{~mL} / \mathrm{min}, 200 \mathrm{bar}, 40^{\circ} \mathrm{C}$
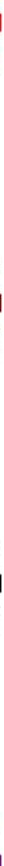
2014-02-11_Leo_A0020
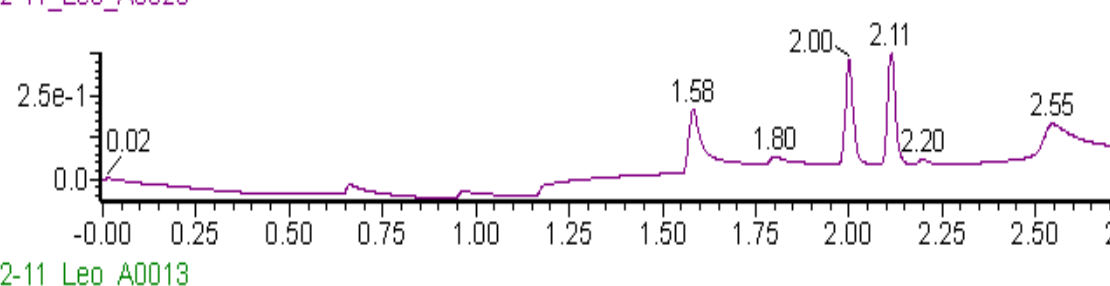

3.68

Chiralcel OZ_MeOH/IBA Range: $4.345 \mathrm{e}-1$ 2014-02-11_Leo_A0013

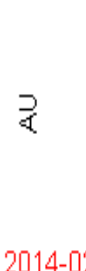

$$
\text { 5.0e-1 }
$$
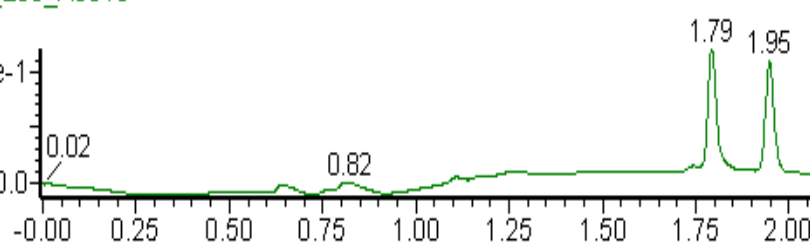

0.82

Chiralcel OJ_MeOH/IBA

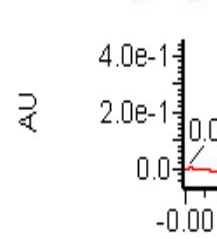
E0_A0006
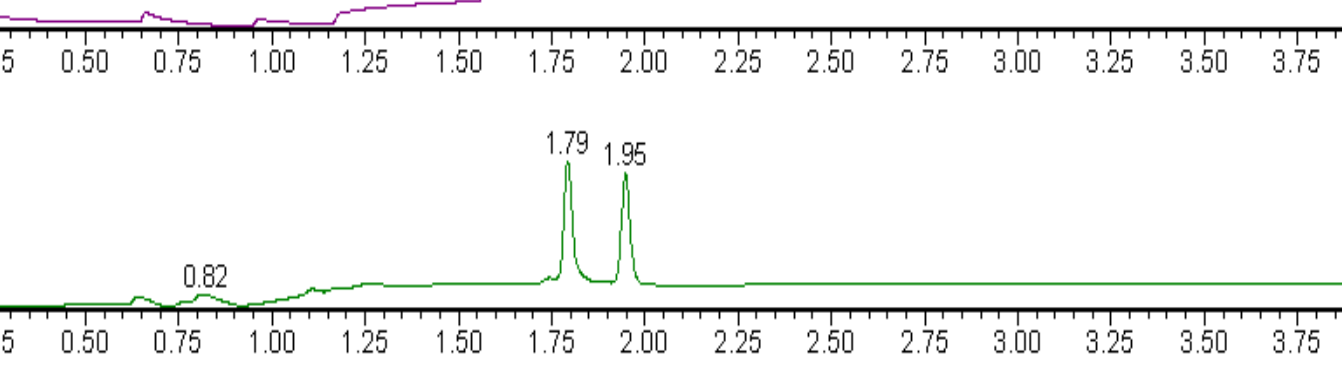

$3 e-1$ 
SFC Screening: 1-40\% (0-5 min), 40\% (5-6 min), no post. $3 \mathrm{~mL} / \mathrm{min}, 200 \mathrm{bar}, 40^{\circ} \mathrm{C}$

\section{S68}
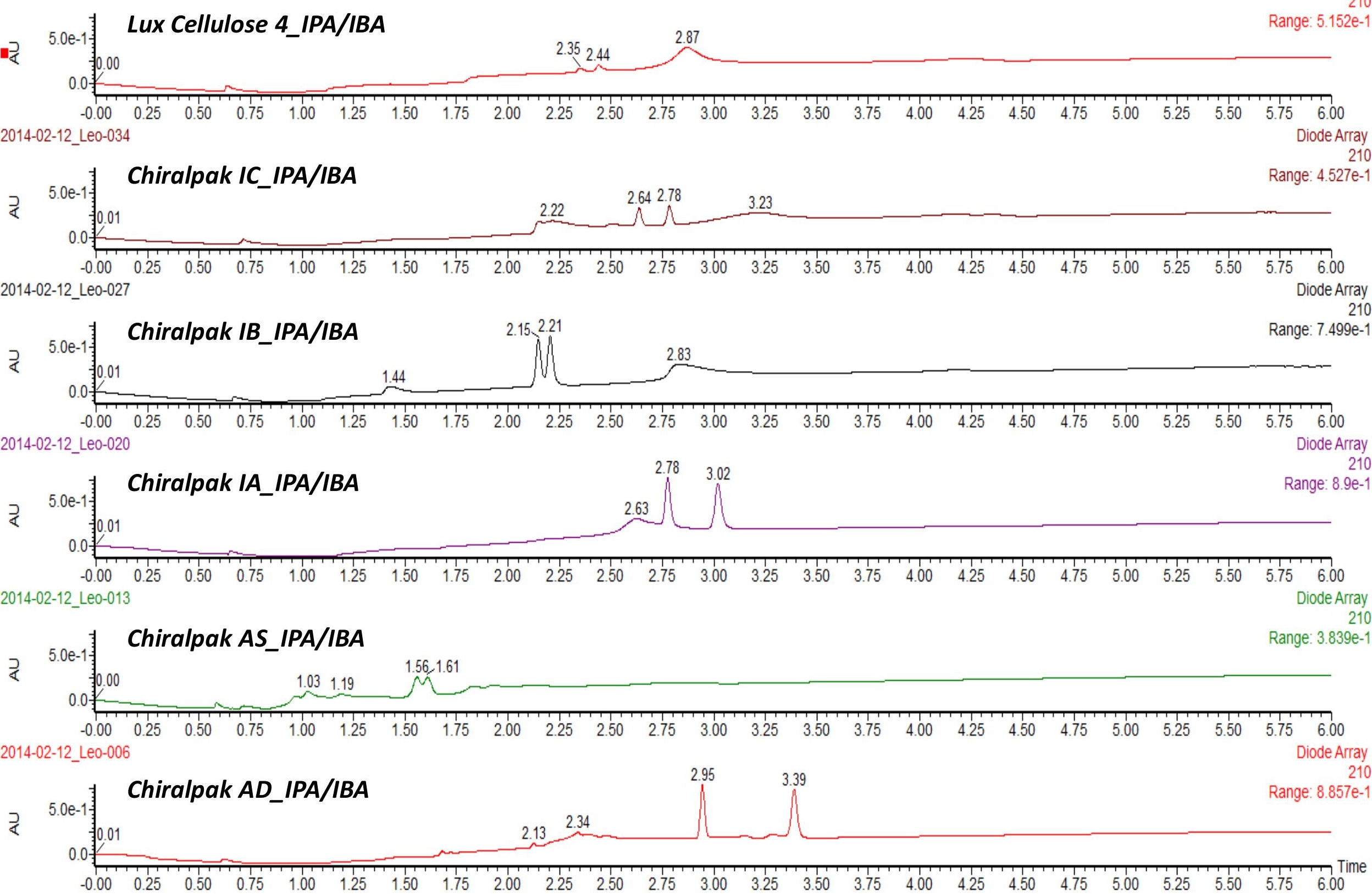
SFC Screening: 1-40\% (0-5 min), 40\% (5-6 min), no post. $3 \mathrm{~mL} / \mathrm{min}, 200 \mathrm{bar}, 40^{\circ} \mathrm{C}$

S69

4-OMe MBA Imine

Diode Array

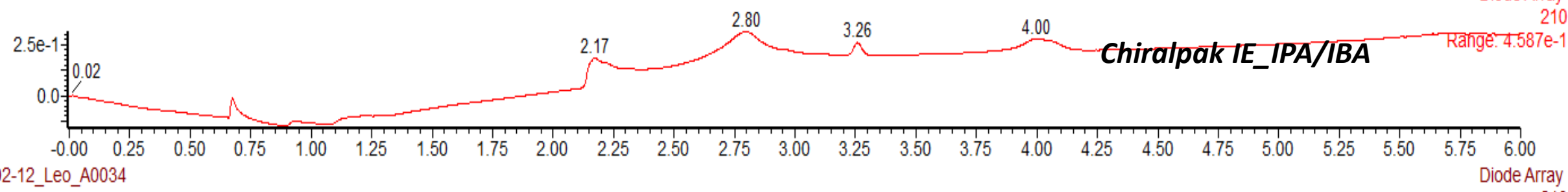

014-02-12_Leo_A0034

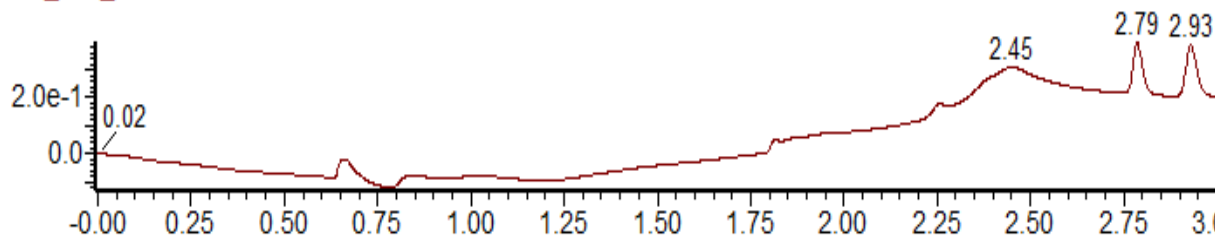

Chiralpak IF_IPA/IBA

Range: $5.18 \mathrm{e}-1$ 2014-02-12_Leo_A0027
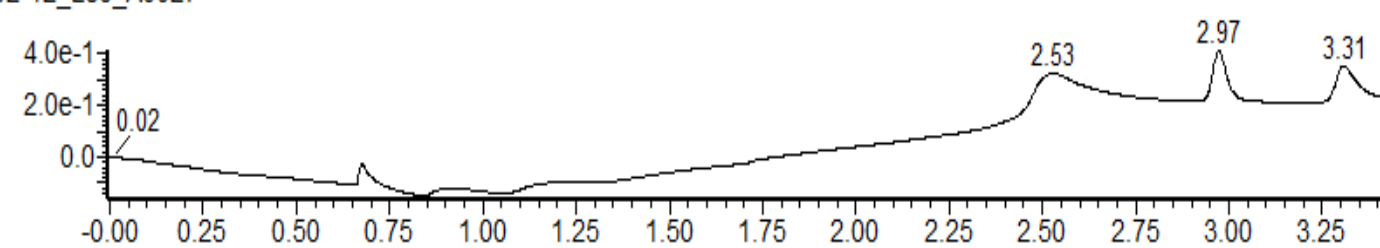

Lux Amylose 2 IPA/IBA

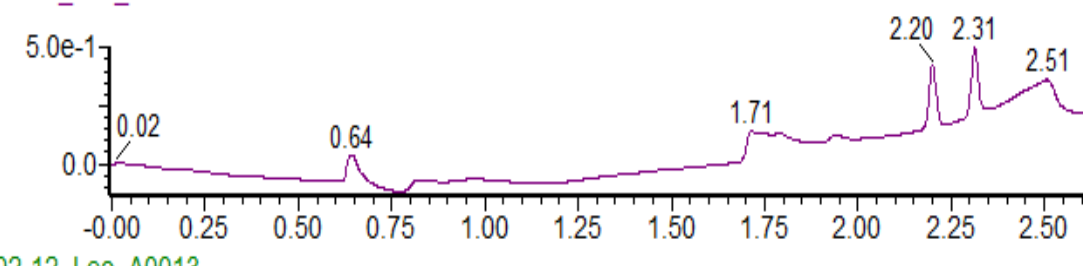
2014-02-12_Leo_A0013

২ 5

5.0e-1
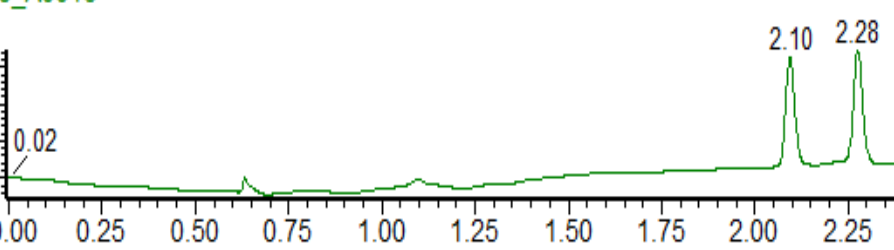

$\frac{2}{4}$

5.0e-1 A0006

Chiralcel OJ_IPA/IBA

\section{Chiralcel OZ_IPA/IBA}

Diode Array

210

$5 \mathrm{e}-1$

ange: $6.234 \mathrm{e}-1$

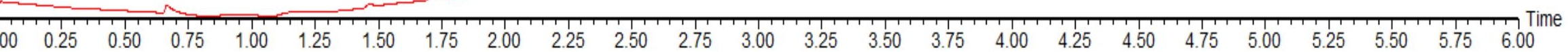




\section{SFC Screening: 1-40\% (0-5 min), 40\% (5-6 min), no post. $3 \mathrm{~mL} / \mathrm{min}, 200 \mathrm{bar}, 40^{\circ} \mathrm{C}$}
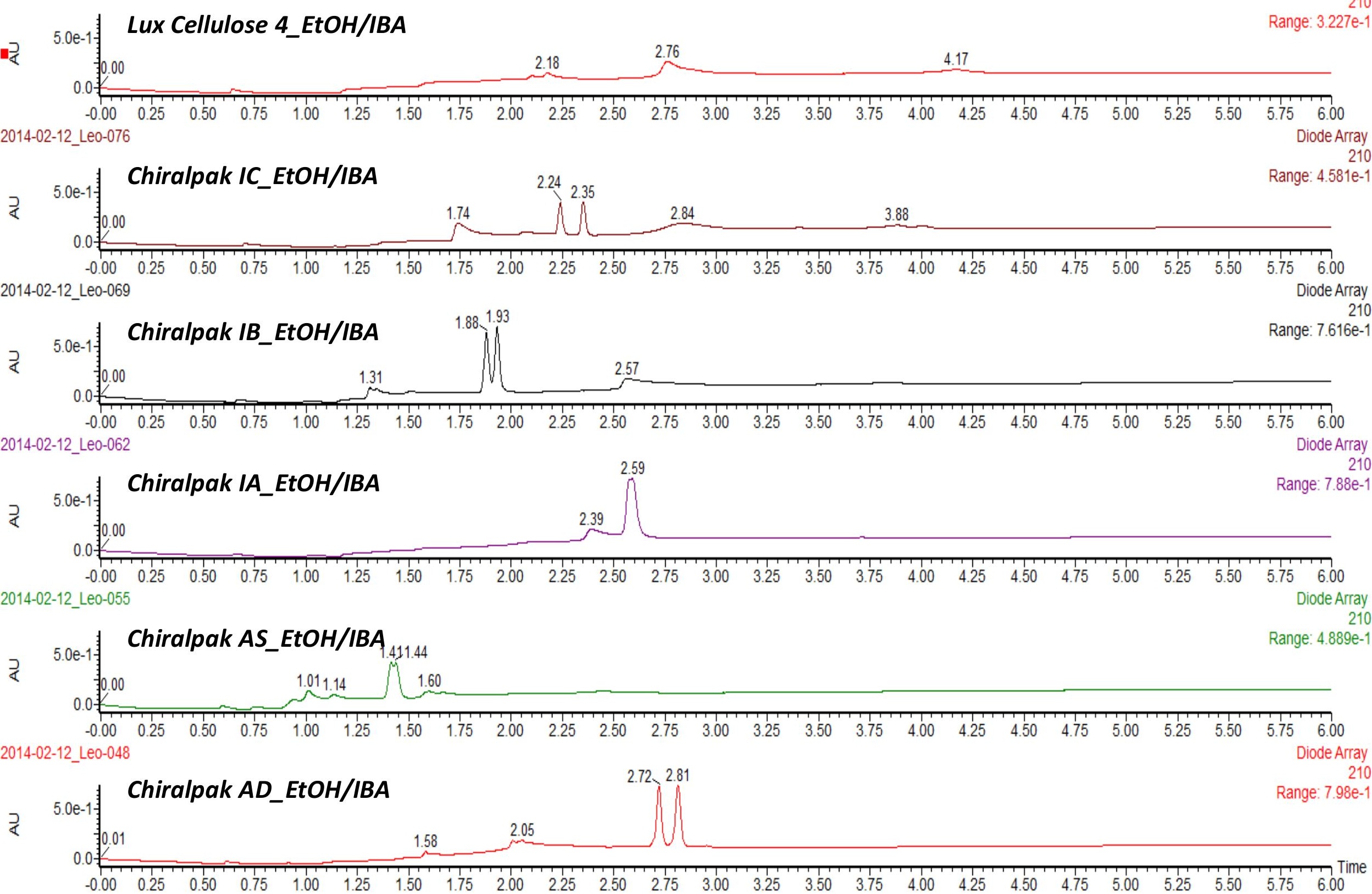


\section{Stereochemistry of Imine Hydrolysis

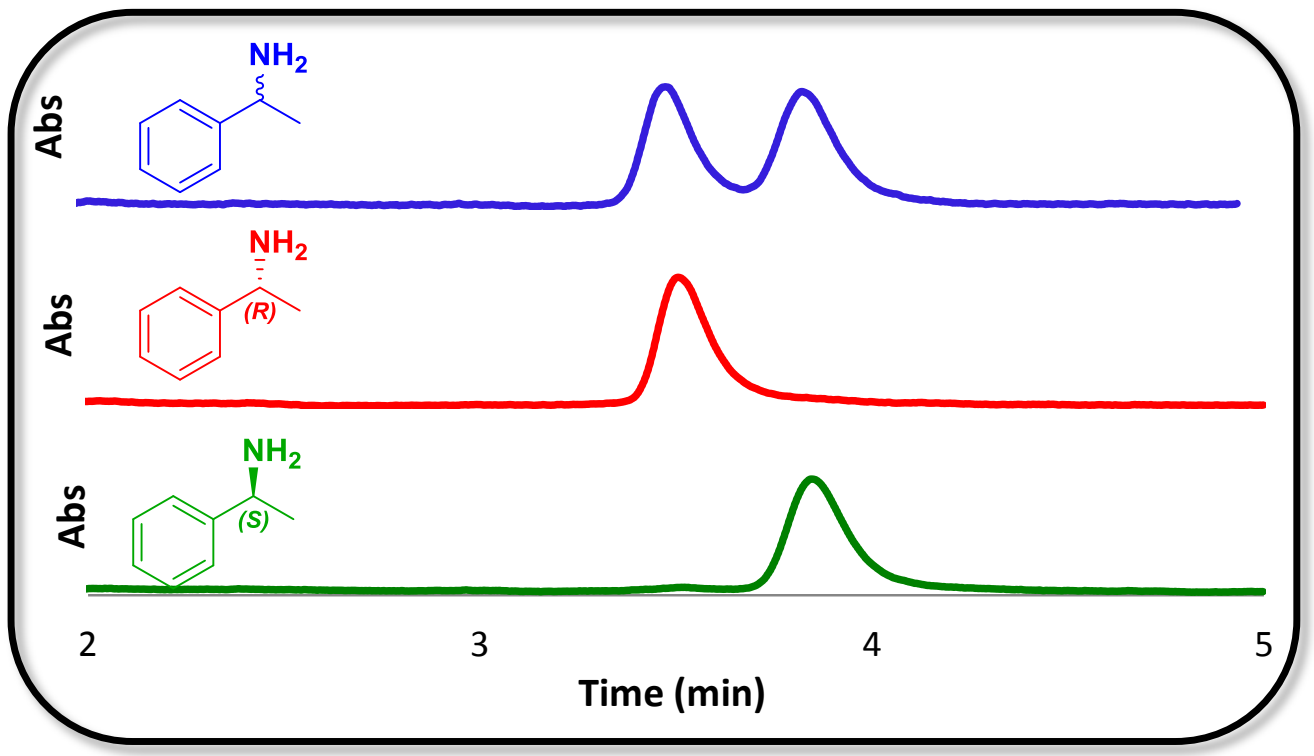

UPC ${ }^{2}$ Method: AD-3 (150x4.6 mm, 3 um); Isocratic: 93\% CO2/7\% EtOH with $25 \mathrm{mM} I B A ; 3 \mathrm{~mL} / \mathrm{min}, 150 \mathrm{bar}, 40^{\circ} \mathrm{C}$.

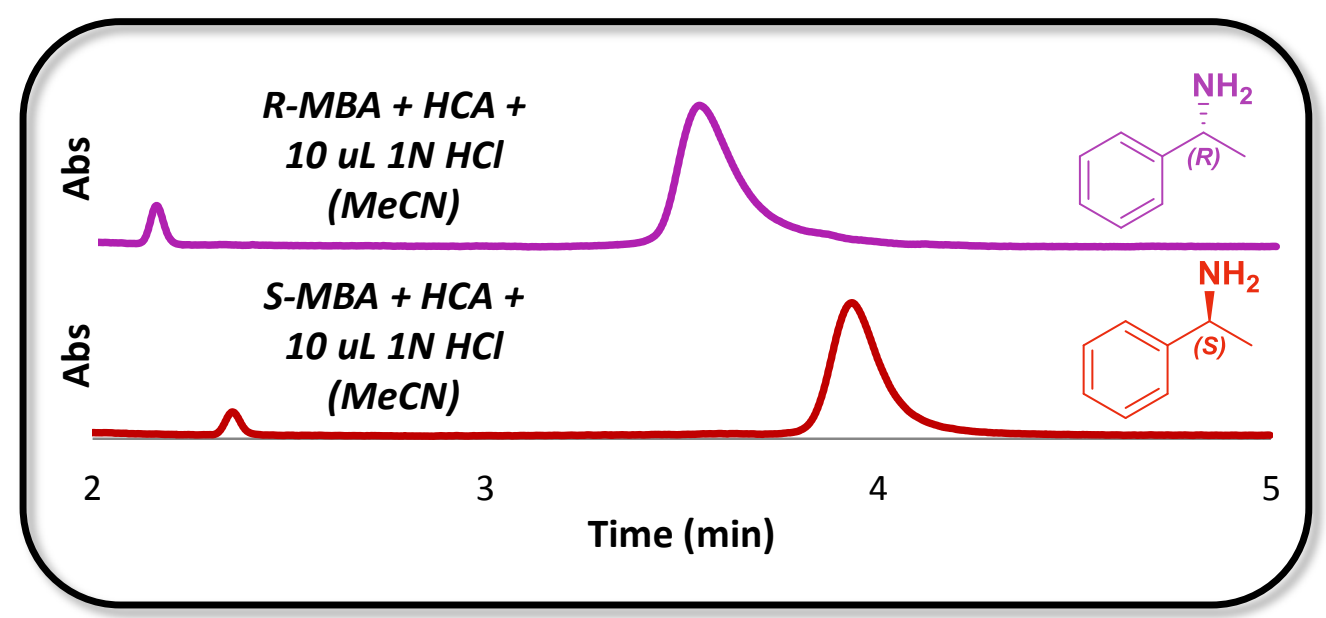

- No racemization during imine hydrolysis 